Supporting Information for:

\title{
Helix-Sense-Selective Encapsulation of Helical Poly(lactic acid)s within a Helical Cavity of Syndiotactic Poly(methyl methacrylate)
}

\section{with Helicity Memory}

\author{
Tomoyuki Ikai, ${ }^{\dagger}$ Satoshi Kawabata, ${ }^{\dagger}$ Fumihiko Mamiya, ${ }^{\dagger}$ Daisuke Taura, ${ }^{\dagger, \neq}$ \\ Naoki Ousaka, ${ }^{\dagger, t, \xi}$ and Eiji Yashima*t,t.
}

\footnotetext{
${ }^{\dagger}$ Department of Molecular and Macromolecular Chemistry, Graduate School of Engineering, Nagoya University, Chikusa-ku, Nagoya 464-8603, Japan

$\ddagger$ Department of Molecular Design and Engineering, Graduate School of Engineering, Nagoya University, Chikusa-ku, Nagoya 464-8603, Japan

$\S$ Present Addresses: Molecular Engineering Institute, Kyushu Institute of Technology, Tobata-ku, Kitakyushu, 804-8550, Japan.
}

E-mail: yashima@chembio.nagoya-u.ac.jp 


\section{Table of Contents}

$\begin{array}{ll}\text { 1. Instruments and Materials } & \text { S3 }\end{array}$

2. Synthetic Procedures $\quad$ S4

3. Preparation of st-PMMA Films Complexed with the $\mathrm{C}_{60}$-Free and $\mathrm{C}_{60}$-Bound $M$ - and $P$-PLAs

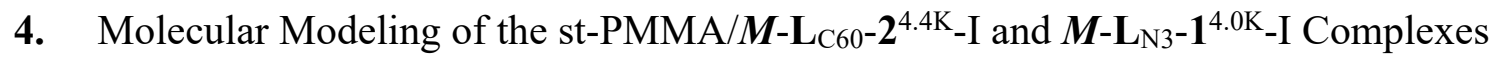

5. General Procedures for VCD Measurements of st-PMMA Gels Complexed with the $\mathrm{C}_{60}$-Free and $\mathrm{C}_{60}$-Bound $M$ - and $P$-PLAs

6. Preparation of st-PMMA $/ M$ - and $P$-PLA Complex Gels in Toluene and Replacement with $\mathrm{C}_{60}$

7. Helix-Sense-Selective Encapsulation of the Racemic $\mathrm{C}_{60}$-Bound and $\mathrm{C}_{60}$-Free $M$ - and $P$-PLAs with $M$ - and $P$-st-PMMA with a Helicity Memory

8. PLA Chain Exchange Reactions Between the $\mathrm{C}_{60}$-Bound Helical $M$ - or $P$-PLAs Encapsulated in the $M$-st-PMMA Helical Cavity and Free $\mathrm{C}_{60}$-Bound Helical $M$ - or $P$-PLAs

9. Helix-Sense-Selective Encapsulation of the Nonracemic $\mathrm{C}_{60}$-Bound PLA with $M$ - and $P$-st-PMMA with a Helicity Memory

10. Supporting Data

11. Supporting References

12. ${ }^{1} \mathrm{H}$ and ${ }^{13} \mathrm{C}$ NMR Spectral Data 


\section{Instruments and Materials.}

Instruments. The NMR spectra were measured using a Bruker Ascend 500 (Bruker Biospin, Billerica, MA) or a Varian 500AS (Agilent Technologies, Santa Clara, CA) spectrometer operating at $500 \mathrm{MHz}$ for ${ }^{1} \mathrm{H}$ and $126 \mathrm{MHz}$ for ${ }^{13} \mathrm{C}$ using tetramethylsilane (TMS) as the internal standard. The electron spray ionization mass spectra (ESI-MS) were recorded on a JEOL JMS-T100CS spectrometer (JEOL, Tokyo, Japan). The matrix-assisted laser desorption-ionization time-of-flight mass spectra (MALDI-TOF-MS) were measured using a Bruker autoflex maX (Bruker Scientific LLC, Billerica, MA) with a positive mode using 1,8,9-anthracenetriol (dithranol) as the matrix. The IR spectra were recorded on a JASCO FT/IR-680 spectrophotometer (JASCO, Tokyo, Japan). The optical rotations were taken using a JASCO P-1030 polarimeter in a 1.0-cm quartz cell equipped with a temperature controller. The absorption and electronic circular dichroism (ECD) spectra were obtained in 0.2-, 0.5-, and 1.0-mm quartz cells using a JASCO V750 spectrophotometer and a JASCO J-1500 spectropolarimeter, respectively. The concentrations of the polymers were calculated based on the monomer units. The temperature was controlled with a JASCO ETCS-900 apparatus. The vibrational circular dichroism (VCD) spectra were measured in a $0.15-\mathrm{mm} \mathrm{BaF}_{2}$ cell with a $\mathrm{JASCO}$ FVS-6000 spectrometer. All spectra were collected for $c a$. $4-5 \mathrm{~h}$ at a resolution of $4 \mathrm{~cm}^{-1}$. The temperature was controlled with a JASCO TCH-FVS apparatus. The differential scanning calorimetry (DSC) measurements were performed on a SEIKO EXSTAR6000 DSC 6200 under nitrogen (Hitachi High-Tech, Tokyo, Japan). The samples were sealed in aluminum pans. The melting temperature $\left(T_{\mathrm{m}}\right)$ and heat of melting $\left(\Delta H_{\mathrm{m}}\right)$ were determined from the minimum of the endothermic peak and by the peak area, respectively. The X-ray diffraction (XRD) measurements were carried out using a Rigaku R-AXIS IV X-ray diffractometer with a rotating-anode generator with graphite monochromated $\mathrm{Cu} K \alpha$ radiation $(\lambda=0.15418 \mathrm{~nm})$ focused through a $0.3 \mathrm{~mm}$ pinhole collimator, which was supplied at $45 \mathrm{kV}$ and $45 \mathrm{~mA}$ current, equipped with a flat imaging plate having a specimen-to-plate distance of $165 \mathrm{~mm}$. The size exclusion chromatography (SEC) measurements were performed with a JASCO PU-4580 liquid chromatograph equipped with a refractive index detector (JASCO RI-4030) and a column oven (JASCO CO-2060). The number-average molar mass $\left(M_{\mathrm{n}}\right)$ and its distribution $\left(M_{\mathrm{w}} / M_{\mathrm{n}}\right)$ were determined at $40{ }^{\circ} \mathrm{C}$ using a Tosoh TSKgel Multipore $\mathrm{H}_{\mathrm{XL}}-\mathrm{M}$ $(30 \mathrm{~cm})$ SEC column (Tosoh, Tokyo, Japan), and chloroform was used as the eluent at a flow rate of $0.5 \mathrm{~mL} / \mathrm{min}$. The molar mass calibration curve was obtained with polystyrene standards (Tosoh). The recycling preparative high-performance liquid chromatography (HPLC) was performed with an LC918 liquid chromatography (JAI, Tokyo, Japan) equipped with a UV detector (JAI UV-310) at room temperature (eluent: chloroform; flow rate $3.5 \mathrm{~mL} / \mathrm{min}$ ). HPLC columns, JAIGEL-1H and JAIGEL$2 \mathrm{H}(60 \mathrm{~cm} \times 2.0 \mathrm{~cm}$ (i.d.)), were connected in series, and chloroform was used as the eluent. 
Materials. The st-PMMA was synthesized by the syndiotactic-specific polymerization of MMA in toluene at $-95^{\circ} \mathrm{C}$ using a typical Ziegler-type catalyst derived from $\mathrm{Al}\left(\mathrm{C}_{2} \mathrm{H}_{5}\right)_{3}$ and $\mathrm{TiCl}_{4} .{ }^{\mathrm{S} 1} \mathrm{The}_{\mathrm{n}}$ and $M_{\mathrm{w}} / M_{\mathrm{n}}$ values and the tacticity (mm:mr:rr) were as follows: $M_{\mathrm{n}}=616,000, M_{\mathrm{w}} / M_{\mathrm{n}}=1.36$, and $m m: m r: r r=0: 4: 96$. The $M_{\mathrm{n}}$ and $M_{\mathrm{w}} / M_{\mathrm{n}}$ values were measured by SEC in chloroform using PMMA standards (Shodex, Tokyo, Japan) for the calibration. The tacticity was determined from the ${ }^{1} \mathrm{H}$ NMR signals due to the $\alpha$-methyl protons. D-Lactide was kindly supplied by Prof. Rong-Ming Ho (National Tsing Hua University, Taiwan) and purified by recrystallization from tetrahydrofuran (THF)/toluene $(3 / 7, \mathrm{v} / \mathrm{v})$ just before use. [60]Fullerene $\left(\mathrm{C}_{60}\right)$ (98\%) was obtained from Frontier Carbon (Tokyo, Japan) and used as received. A fullerene derivative (4) was synthesized according to the reported procedure. ${ }^{\mathrm{S} 2}$ All starting materials and anhydrous solvents were purchased from Sigma-Aldrich (St. Louis, MO), Wako Pure Chemical Industries (Osaka, Japan), Kokusan Chemical Co. Ltd. (Tokyo, Japan), and Tokyo Kasei (Tokyo, Japan) unless otherwise noted.

\section{Synthetic Procedures}

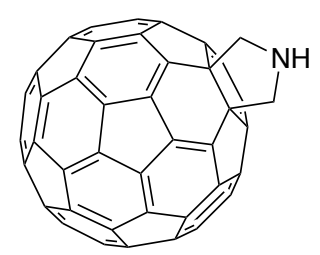

4

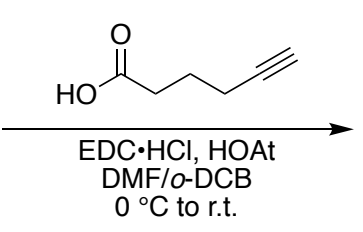

$0{ }^{\circ} \mathrm{C}$ to r.t.

Scheme S1. Synthesis of E-C 60 .

Synthesis of E-C $\mathbf{6}_{\mathbf{6 0}}$. To a mixture of 7-aza-1-hydroxy-1,2,3-benzotriazole (HOAt) (25 mg, 0.18 $\mathrm{mmol}$ ) and 1-ethyl-3-(3-dimethylaminopropyl)-carbodiimide hydrochloride (EDC $\cdot \mathrm{HCl})(29 \mathrm{mg}, 0.15$ $\mathrm{mmol})$ in anhydrous dichloromethane $(1.3 \mathrm{~mL})$ was added 5-hexynoic acid $(17 \mu \mathrm{L}, 0.15 \mathrm{mmol})$. The solution was degassed with three freeze-pump-thaw cycles and stirred at $0{ }^{\circ} \mathrm{C}$ under nitrogen for 30 min. To this was then added a degassed solution of the fullerene derivative 4 (79 $\mathrm{mg}, 0.10 \mathrm{mmol})$ in $o$-dichlorobenzene $(1.3 \mathrm{~mL})$ at $0{ }^{\circ} \mathrm{C}$ under nitrogen, and the reaction mixture was stirred at room temperature for $24 \mathrm{~h}$. After removal of the solvents under reduced pressure, the residue was dissolved in chloroform, and the solution was washed with $1 \mathrm{~N}$ aqueous $\mathrm{HCl}, 5 \%$ aqueous $\mathrm{NaHCO}_{3}$, and brine, and then dried over $\mathrm{MgSO}_{4}$. Purification by column chromatography on silica gel using 
chloroform/EtOAc (9/1, v/v) as the eluent and further reprecipitation from chloroform to diethyl ether afforded $\mathrm{E}_{-\mathrm{C}} \mathrm{C}_{60}\left(36 \mathrm{mg}, 42 \%\right.$ yield) as a brown solid. HRMS (ESI+): $\mathrm{m} / z$ calcd for $\mathrm{C}_{68} \mathrm{H}_{11} \mathrm{NNaO}$ $\left(\mathrm{M}+\mathrm{Na}^{+}\right), 880.0733$; found 880.0739. ${ }^{1} \mathrm{H} \mathrm{NMR}\left(500 \mathrm{MHz}, o-\mathrm{DCB}-d_{4}, 25{ }^{\circ} \mathrm{C}\right): \delta 5.66(\mathrm{~s}, 2 \mathrm{H}), 5.46(\mathrm{~s}$, $2 \mathrm{H}$ ), $3.16(\mathrm{t}, J=7.0 \mathrm{~Hz}, 2 \mathrm{H}), 2.72(\mathrm{dt}, J=2.5,7.0 \mathrm{~Hz}, 2 \mathrm{H}), 2.42$ (quint, $J=7.0 \mathrm{~Hz}, 2 \mathrm{H}$ ), 2.33 (dt, $J$ $=0.5,3.0 \mathrm{~Hz}, 1 \mathrm{H}) .{ }^{13} \mathrm{C} \mathrm{NMR}\left(126 \mathrm{MHz}, o\right.$-DCB- $\left.d_{4}, 25{ }^{\circ} \mathrm{C}\right): \delta 170.47,153.84,153.23,147.38,146.34$, 146.12 , 145.55, 145.51, 145.33, 144.46, 143.06, 142.66, 142.25, 142.09, 141.92, 140.23, 136.21, 135.73, 84.02, 71.26, 69.97, 69.71, 59.22, 57.58, 33.00, 24.34, 18.40. Mp: $135^{\circ} \mathrm{C}$ (dec.). IR (KBr, $\left.\mathrm{cm}^{-1}\right): 1637\left(\nu_{\mathrm{C}=0}\right)$.

Synthesis of $\boldsymbol{M}-\mathbf{N}_{3}-$ PLLA and $\boldsymbol{P}-\mathbf{N}_{3}$-PDLA. $\mathrm{N}_{3}$-Polylactides $\left(\boldsymbol{M}-\mathrm{N}_{3}-\mathrm{PLLA}\right.$ and $\left.\boldsymbol{P}-\mathrm{N}_{3}-\mathrm{PDLA}\right)$ were prepared by ring-opening polymerization of L- and D-lactides initiated with 3-azido-1-propanol in the presence of 1,8-diazabicyclo[5.4.0]-7-undecene (DBU) as a catalyst, respectively, in a similar way as previously reported. ${ }^{\mathrm{S} 3}$

A typical procedure for the polymerization of D-lactide is described as follows. To a solution of D-lactide $(0.30 \mathrm{~g}, 2.1 \mathrm{mmol})$ in anhydrous dichloromethane $(2.1 \mathrm{~mL})$ were added DBU $(5.2 \mu \mathrm{L}, 35$ $\mu \mathrm{mol})$ and 3-azido-1-propanol $(28 \mu \mathrm{L}, 0.30 \mathrm{mmol})$ at room temperature under nitrogen to initiate the polymerization. After $10 \mathrm{~min}$, the polymerization was quenched by the addition of benzoic acid (30 $\mathrm{mg}, 0.25 \mathrm{mmol})$. The mixture was purified by reprecipitation from dichloromethane into cold methanol to give $\boldsymbol{P}-\mathrm{N}_{3}-\mathrm{PDLA}^{0.9 \mathrm{~K}}(0.27 \mathrm{~g}, 91 \%$ yield$)$ as a white solid. In the same way, $\boldsymbol{P}-\mathrm{N}_{3}-\mathrm{PDLA}$ and $\boldsymbol{M}$ - $\mathrm{N}_{3}$-PLLA with different molar masses were prepared. The $M_{\mathrm{n}}$ and $M_{\mathrm{w}} / M_{\mathrm{n}}$ values of the polymers were determined by SEC using polystyrene standards (Shodex, Tokyo, Japan) in chloroform. The $M_{\mathrm{n}}$ values were also estimated by ${ }^{1} \mathrm{H}$ NMR azido end-group analysis $\left(M_{\mathrm{n}, \mathrm{NMR}}\right)$ in $\mathrm{CDCl}_{3}$. The polymerization results are summarized in Table S1. 
Table S1. Polymerization of $\mathrm{L}$ - and D-Lactides Initiated by 3-Azido-1-Propanol with $\mathrm{DBU}$ in $\mathrm{CH}_{2} \mathrm{Cl}_{2}$ at Room Temperature for 10 min $^{a}$
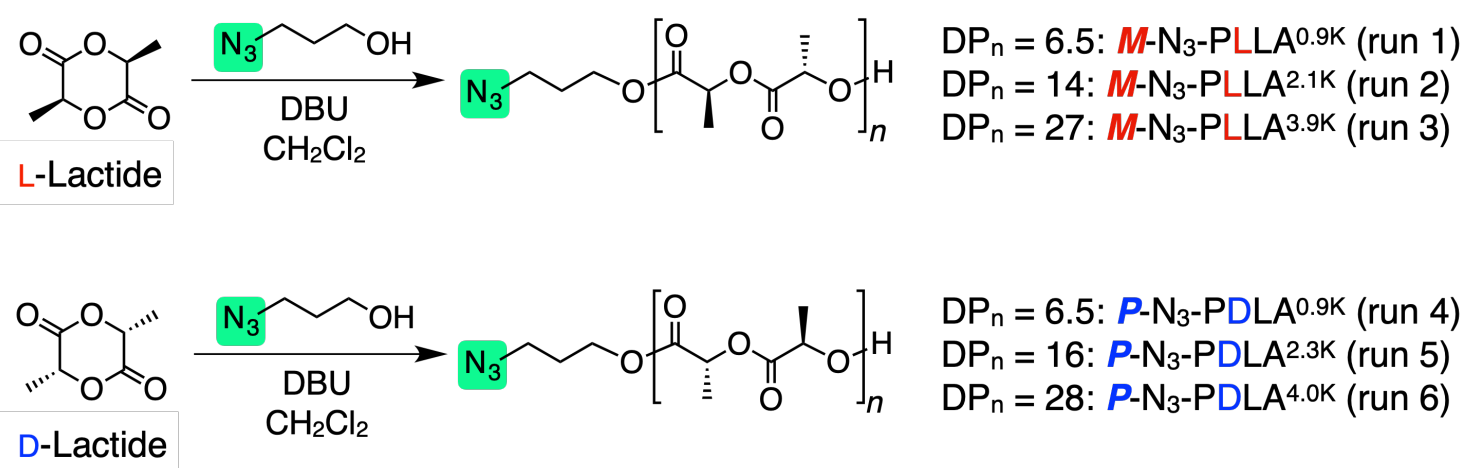

\begin{tabular}{|c|c|c|c|c|c|c|c|c|c|c|}
\hline \multirow[b]{2}{*}{ run } & \multirow[b]{2}{*}{ monomer } & \multirow{2}{*}{$\begin{array}{c}\text { [monomer }] / \\
\text { [initiator] }\end{array}$} & \multicolumn{8}{|c|}{ PLA prepolymer } \\
\hline & & & sample code & $\begin{array}{c}\text { yield } \\
(\%) \\
\end{array}$ & $\begin{array}{l}M_{\mathrm{n}, \mathrm{SEC}} \\
\left(10^{3}\right)^{b} \\
\end{array}$ & $M_{\mathrm{w}} / M_{\mathrm{n}}{ }^{b}$ & $\begin{array}{l}M_{\mathrm{n}, \mathrm{NMR}} \\
\left(10^{3}\right)^{c} \\
\end{array}$ & $\mathrm{DP}_{\mathrm{n}, \mathrm{NMR}}{ }^{d}$ & {$[\alpha]_{\mathrm{D}}^{25 e}$} & $\begin{array}{c}T_{\mathrm{m}} \\
\left({ }^{\circ} \mathrm{C}\right)^{f}\end{array}$ \\
\hline 1 & L-lactide & 7 & $\boldsymbol{M}-\mathrm{N}_{3}-\mathrm{PLLA}^{0.9 \mathrm{~K}}$ & 87 & 1.53 & 1.26 & 0.94 & 6.5 & -121.7 & 92 \\
\hline 2 & L-lactide & 14 & $\boldsymbol{M}-\mathrm{N}_{3}-\mathrm{PLLA}^{2.1 \mathrm{~K}}$ & 74 & 3.55 & 1.11 & 2.08 & 14 & -132.0 & 130 \\
\hline 3 & L-lactide & 26 & $\boldsymbol{M}-\mathrm{N}_{3}-\mathrm{PLLA}^{3.9 \mathrm{~K}}$ & 87 & 6.28 & 1.11 & 3.85 & 27 & -140.9 & 151 \\
\hline 4 & D-lactide & 7 & $\boldsymbol{P}-\mathrm{N}_{3}-\mathrm{PDLA}^{0.9 \mathrm{~K}}$ & 91 & 1.40 & 1.31 & 0.94 & 6.5 & +124.9 & 93 \\
\hline 5 & D-lactide & 15 & $\boldsymbol{P}-\mathrm{N}_{3}-\mathrm{PDLA}^{2.3 \mathrm{~K}}$ & 90 & 3.82 & 1.12 & 2.28 & 16 & +134.0 & 136 \\
\hline 6 & D-lactide & 29 & $\boldsymbol{P}-\mathrm{N}_{3}-\mathrm{PDLA}^{4.0 \mathrm{~K}}$ & 78 & 7.11 & 1.12 & 4.04 & 28 & +139.1 & 152 \\
\hline
\end{tabular}

${ }^{a}[$ Monomer $]=1.0 \mathrm{M},[\mathrm{DBU}] /[$ monomer $]=0.01$. The complete consumption of monomers was confirmed by ${ }^{1} \mathrm{H}$ NMR analysis in each run. ${ }^{b}$ Estimated by SEC (polystyrene standards) with $\mathrm{CHCl}_{3}$ as the eluent. ${ }^{c}$ Determined by the ${ }^{1} \mathrm{H}$ NMR end-group analysis in $\mathrm{CDCl}_{3} .{ }^{d}$ Number-average degree of polymerization determined by $\mathrm{MnNMR}_{\mathrm{n},{ }^{e}}$ Measured in $\mathrm{CHCl}_{3}$ at $25^{\circ} \mathrm{C} .{ }^{f}$ Melting point determined by DSC. 
Spectroscopic data of $\boldsymbol{M}$ - $\mathrm{N}_{3}-\mathrm{PLLA}^{0.9 \mathrm{~K}}: \mathrm{IR}\left(\mathrm{KBr}, \mathrm{cm}^{-1}\right): 2103(\mathrm{~N}=\mathrm{N}=\mathrm{N}), 1759(\mathrm{C}=\mathrm{O}), 1191(\mathrm{C}-\mathrm{O})$, $1094(\mathrm{C}-\mathrm{O}) .{ }^{1} \mathrm{H}$ NMR $\left(500 \mathrm{MHz}, \mathrm{CDCl}_{3}, 25^{\circ} \mathrm{C}\right): \delta 5.24-5.10(\mathrm{~m}, \mathrm{CH}$ PLA backbone, $12 \mathrm{H}), 4.36(\mathrm{q}$, $J=7.0 \mathrm{~Hz}, \mathrm{CH}$ PLA backbone, $1 \mathrm{H}), 4.24\left(\mathrm{dt}, J=6.2,2.8 \mathrm{~Hz}, \mathrm{OCH}_{2}, 2 \mathrm{H}\right), 3.39\left(\mathrm{t}, J=6.7 \mathrm{~Hz}, \mathrm{~N}_{3} \mathrm{CH}_{2}\right.$, 2H), 2.64 (br, OH, 1H), 1.94-1.89 (m, $\left.\mathrm{OCH}_{2} \mathrm{CH}_{2}, 2 \mathrm{H}\right), 1.61-1.49$ (m, $\mathrm{CH}_{3}$ PLA backbone, partially overlapping with $\mathrm{H}_{2} \mathrm{O}$ signal) (for all the peak assignments including the end groups, see Figure S2a(i)).

Spectroscopic data of $\boldsymbol{M}$-N $3-\mathrm{PLLA}^{2.1 \mathrm{~K}}$ : IR $\left(\mathrm{KBr}, \mathrm{cm}^{-1}\right): 2103(\mathrm{~N}=\mathrm{N}=\mathrm{N}), 1759(\mathrm{C}=\mathrm{O}), 1185(\mathrm{C}-\mathrm{O})$, $1092(\mathrm{C}-\mathrm{O}) .{ }^{1} \mathrm{H}$ NMR $\left(500 \mathrm{MHz}, \mathrm{CDCl}_{3}, 25^{\circ} \mathrm{C}\right): \delta 5.24-5.10(\mathrm{~m}, \mathrm{CH}$ PLA backbone, $28 \mathrm{H}), 4.35$ (q, $J=7.0 \mathrm{~Hz}, \mathrm{CH}$ PLA backbone, $1 \mathrm{H}), 4.24\left(\mathrm{dt}, J=6.0,3.0 \mathrm{~Hz}, \mathrm{OCH}_{2}, 2 \mathrm{H}\right), 3.39\left(\mathrm{t}, J=6.7 \mathrm{~Hz}, \mathrm{~N}_{3} \mathrm{CH}_{2}\right.$, 2H), 2.65 (br, OH, 1H), 1.94-1.89 (m, $\left.\mathrm{OCH}_{2} \mathrm{CH}_{2}, 2 \mathrm{H}\right), 1.61-1.49$ (m, $\mathrm{CH}_{3}$ PLA backbone, partially overlapping with $\mathrm{H}_{2} \mathrm{O}$ signal) (for all the peak assignments including the end groups, see Figure S3a(i)).

Spectroscopic data of $\boldsymbol{M}$ - $\mathrm{N}_{3}-\mathrm{PLLA}^{3.9 \mathrm{~K}}$ : IR $\left(\mathrm{KBr}, \mathrm{cm}^{-1}\right): 2103(\mathrm{~N}=\mathrm{N}=\mathrm{N}), 1759(\mathrm{C}=\mathrm{O}), 1185(\mathrm{C}-\mathrm{O})$, $1092(\mathrm{C}-\mathrm{O}) .{ }^{1} \mathrm{H}$ NMR $\left(500 \mathrm{MHz}, \mathrm{CDCl}_{3}, 25^{\circ} \mathrm{C}\right): \delta 5.24-5.10(\mathrm{~m}, \mathrm{CH}$ PLA backbone, 52H), 4.35 (q, $J=6.9 \mathrm{~Hz}, \mathrm{CH}$ PLA backbone, $1 \mathrm{H}), 4.24\left(\mathrm{dt}, J=6.0,3.0 \mathrm{~Hz}, \mathrm{OCH}_{2}, 2 \mathrm{H}\right), 3.38\left(\mathrm{t}, J=6.7 \mathrm{~Hz}, \mathrm{~N}_{3} \mathrm{CH}_{2}\right.$, 2H), 2.65 (br, OH, 1H), 1.94-1.89 (m, $\left.\mathrm{OCH}_{2} \mathrm{CH}_{2}, 2 \mathrm{H}\right), 1.61-1.49$ (m, $\mathrm{CH}_{3}$ PLA backbone, partially overlapping with $\mathrm{H}_{2} \mathrm{O}$ signal) (for all the peak assignments including the end groups, see Figure 2a(i)).

Spectroscopic data of $\boldsymbol{P}-\mathrm{N}_{3}-\mathrm{PDLA}{ }^{0.9 \mathrm{~K}}$ : IR $\left(\mathrm{KBr}, \mathrm{cm}^{-1}\right): 2103(\mathrm{~N}=\mathrm{N}=\mathrm{N}), 1760(\mathrm{C}=\mathrm{O}), 1190(\mathrm{C}-\mathrm{O})$, $1094(\mathrm{C}-\mathrm{O}) .{ }^{1} \mathrm{H}$ NMR $\left(500 \mathrm{MHz}, \mathrm{CDCl}_{3}, 25^{\circ} \mathrm{C}\right): \delta 5.24-5.10(\mathrm{~m}, \mathrm{CH}$ PLA backbone, 12H), 4.36 (q, $J=6.8 \mathrm{~Hz}, \mathrm{CH}$ PLA backbone, $1 \mathrm{H}), 4.24\left(\mathrm{dt}, J=6.3,2.8 \mathrm{~Hz}, \mathrm{OCH}_{2}, 2 \mathrm{H}\right), 3.38\left(\mathrm{t}, J=6.7 \mathrm{~Hz}, \mathrm{~N}_{3} \mathrm{CH}_{2}\right.$, $2 \mathrm{H}), 2.65(\mathrm{~d}, J=6.0 \mathrm{~Hz}, \mathrm{OH}, 1 \mathrm{H}), 1.94-1.89\left(\mathrm{~m}, \mathrm{OCH}_{2} \mathrm{CH}_{2}, 2 \mathrm{H}\right), 1.61-1.49$ (m, $\mathrm{CH}_{3}$ PLA backbone, partially overlapping with $\mathrm{H}_{2} \mathrm{O}$ signal) (for all the peak assignments including the end groups, see Figure S4a(i)).

Spectroscopic data of $\boldsymbol{P}-\mathrm{N}_{3}-\mathrm{PDLA}{ }^{2.3 \mathrm{~K}}$ : IR $\left(\mathrm{KBr}, \mathrm{cm}^{-1}\right): 2101(\mathrm{~N}=\mathrm{N}=\mathrm{N}), 1759(\mathrm{C}=\mathrm{O}), 1186(\mathrm{C}-\mathrm{O})$, $1093(\mathrm{C}-\mathrm{O}) .{ }^{1} \mathrm{H}$ NMR $\left(500 \mathrm{MHz}, \mathrm{CDCl}_{3}, 25^{\circ} \mathrm{C}\right): \delta 5.24-5.10(\mathrm{~m}, \mathrm{CH}$ PLA backbone, 31H), 4.36 (q, $J=6.9 \mathrm{~Hz}, \mathrm{CH}$ PLA backbone, $1 \mathrm{H}), 4.24\left(\mathrm{dt}, J=6.0,3.0 \mathrm{~Hz}, \mathrm{OCH}_{2}, 2 \mathrm{H}\right), 3.39\left(\mathrm{t}, J=6.7 \mathrm{~Hz}, \mathrm{~N}_{3} \mathrm{CH}_{2}\right.$, 2H), 2.65 (br, OH, 1H), 1.94-1.89 (m, $\left.\mathrm{OCH}_{2} \mathrm{CH}_{2}, 2 \mathrm{H}\right), 1.61-1.49$ (m, $\mathrm{CH}_{3}$ PLA backbone, partially overlapping with $\mathrm{H}_{2} \mathrm{O}$ signal) (for all the peak assignments including the end groups, see Figure S5a(i)).

Spectroscopic data of $\boldsymbol{P}-\mathrm{N}_{3}-\mathrm{PDLA}{ }^{4.0 \mathrm{~K}}$ : IR $\left(\mathrm{KBr}, \mathrm{cm}^{-1}\right): 2103(\mathrm{~N}=\mathrm{N}=\mathrm{N}), 1759(\mathrm{C}=\mathrm{O}), 1185(\mathrm{C}-\mathrm{O})$, $1091(\mathrm{C}-\mathrm{O}) .{ }^{1} \mathrm{H}$ NMR $\left(500 \mathrm{MHz}, \mathrm{CDCl}_{3}, 25^{\circ} \mathrm{C}\right): \delta 5.24-5.10(\mathrm{~m}, \mathrm{CH}$ PLA backbone, 55H), 4.36 (q, 
$J=7.0 \mathrm{~Hz}, \mathrm{CH}$ PLA backbone, $1 \mathrm{H}), 4.24\left(\mathrm{dt}, J=6.0,3.0 \mathrm{~Hz}, \mathrm{OCH}_{2}, 2 \mathrm{H}\right), 3.38\left(\mathrm{t}, J=6.5 \mathrm{~Hz}, \mathrm{~N}_{3} \mathrm{CH}_{2}\right.$, 2H), 2.65 (br, OH, 1H), 1.94-1.89 (m, $\left.\mathrm{OCH}_{2} \mathrm{CH}_{2}, 2 \mathrm{H}\right), 1.61-1.49$ (m, $\mathrm{CH}_{3}$ PLA backbone, partially overlapping with $\mathrm{H}_{2} \mathrm{O}$ signal) (for all the peak assignments including the end groups, see Figure S6a(i)).

Synthesis of $\boldsymbol{M}-\mathrm{L}_{\mathrm{N} 3}-1-\mathrm{I}$ and $\boldsymbol{P}-\mathrm{D}_{\mathrm{N} 3}-1-\mathrm{F} . \boldsymbol{M}-\mathrm{L}_{\mathrm{N} 3}-1-\mathrm{I}$ and $\boldsymbol{P}-\mathrm{D}_{\mathrm{N} 3}-1-\mathrm{F}$ were prepared by esterification of the terminal hydroxy groups of $\boldsymbol{M}$ - $\mathbf{N}_{3}$-PLLA and $\boldsymbol{P}-\mathbf{N}_{3}$-PDLA with 4-iodo- and 4-fluoroiodobenzoyl chlorides, respectively.

A typical procedure for the esterification of $\boldsymbol{P}-\mathrm{N}_{3}-\mathrm{PDLA}^{1.0 \mathrm{~K}}$ is described as follows. To a solution of $\boldsymbol{P}-\mathrm{N}_{3}-\mathrm{PDLA}^{1.0 \mathrm{~K}}(0.20 \mathrm{~g}, 0.19 \mathrm{mmol})$ in anhydrous dichloromethane/pyridine $(3 / 1, \mathrm{v} / \mathrm{v} ; 2.7 \mathrm{~mL})$ was added 4-fluorobenzoyl chloride $(0.67 \mathrm{~mL}, 5.6 \mathrm{mmol})$. After the reaction mixture was stirred at room temperature for $17 \mathrm{~h}$ under nitrogen, the solution was concentrated to ca. $0.5 \mathrm{~mL}$. The residue was precipitated into methanol $(2 \mathrm{~mL})$, collected by centrifugation, and washed with methanol to afford $\boldsymbol{P}$ - $\mathbf{D}_{\mathrm{N} 3}-\mathbf{1}^{1.0 \mathrm{~K}}-\mathrm{F}(0.17 \mathrm{~g}, 72 \%$ yield $)$ as a white solid. In the same way, $\boldsymbol{P}-\mathbf{D}_{\mathrm{N} 3}-\mathbf{1}-\mathrm{F}$ and $\boldsymbol{M}-\mathbf{L}_{\mathrm{N} 3}-\mathbf{1}-$ I and with different molar masses were prepared. The $M_{\mathrm{n}}$ and $M_{\mathrm{w}} / M_{\mathrm{n}}$ values of the polymers were determined by SEC using polystyrene standards in chloroform. The $M_{\mathrm{n}}$ values were also estimated by ${ }^{1} \mathrm{H}$ NMR end-group analysis $\left(M_{\mathrm{n}, \mathrm{NMR}}\right)$ in $\mathrm{CDCl}_{3}$. The esterification results are summarized in Table $\mathrm{S} 2$. 
Table S2. Esterification of $M$ - $\mathrm{N}_{3}$-PLLA and $P$ - $\mathrm{N}_{3}$-PDLA with 4-Iodo- and 4-Fluorobenzoyl Chloride, respectively, in $\mathrm{CH}_{2} \mathrm{Cl}_{2}$ at Room Temperature
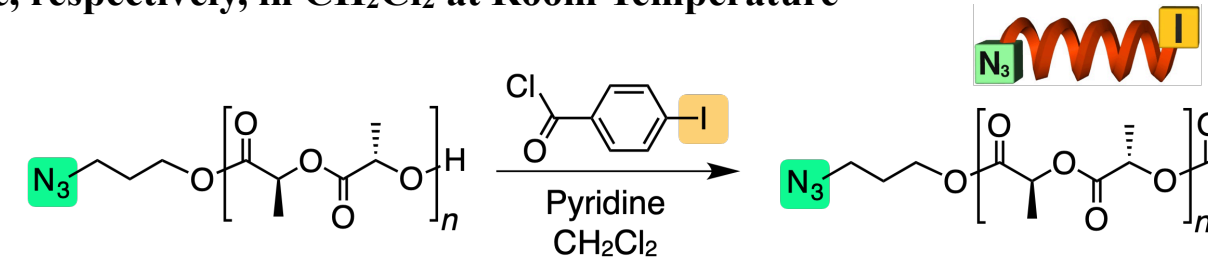

$$
\begin{aligned}
& \mathrm{DP}_{\mathrm{n}}=6.5: M-\mathrm{N}_{3}-\mathrm{PLLA}^{0.9 \mathrm{~K}}(\text { run } 1) \\
& \mathrm{DP}_{\mathrm{n}}=14: M-\mathrm{N}_{3}-\mathrm{PLLA}^{2.1 \mathrm{~K}}(\text { run 2) } \\
& \mathrm{DP}_{\mathrm{n}}=27: M-\mathrm{N}_{3}-\mathrm{PLLA}^{3.9 \mathrm{~K}}(\text { run 3) }
\end{aligned}
$$

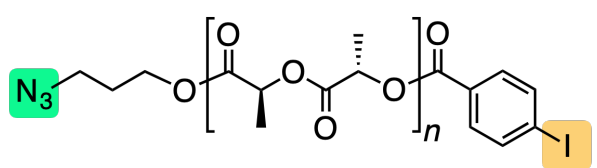

$$
\begin{aligned}
& D P_{n}=6.6: M-L_{N 3}-1^{1.0 K}-I \text { (run 1) } \\
& D P_{n}=16: M-L_{N 3}-12.3 K-I \text { (run 2) } \\
& D P_{n}=28: M-L_{N} 3^{-14.0 K-I ~(r u n ~ 3) ~}
\end{aligned}
$$

\section{s]l $\mathrm{N}_{\mathrm{F}}$}<smiles>CC(C)OC(=O)C(C)OC(=O)C(C)OCCCN</smiles>

$\mathrm{DP}_{\mathrm{n}}=6.5: P-\mathrm{N}_{3}-\mathrm{PDLA} 0.9 \mathrm{~K}$ (run 4)

$\mathrm{DP}_{\mathrm{n}}=16: P-\mathrm{N}_{3}-\mathrm{PDLA}$ 2.3K (run 5)

$\mathrm{DP}_{\mathrm{n}}=28: \mathrm{P}^{-\mathrm{N}_{3}-\mathrm{PDLA}} \mathrm{A}^{4.0 \mathrm{~K}}$ (run 6)

\begin{tabular}{|c|c|c|c|c|c|c|c|c|c|}
\hline run & reactant & product & $\begin{array}{l}\text { yield } \\
(\%)^{a}\end{array}$ & $\begin{array}{l}M_{\mathrm{n}, \mathrm{SEC}} \\
\left(10^{3}\right)^{b} \\
\end{array}$ & $M_{\mathrm{w}} / M_{\mathrm{n}}{ }^{b}$ & $\begin{array}{c}M_{\mathrm{n}, \mathrm{NMR}} \\
\left(10^{3}\right)^{c} \\
\end{array}$ & $\mathrm{DP}_{\mathrm{n}, \mathrm{NMR}}{ }^{d}$ & $\begin{array}{c}f_{\text {benzoate }} \\
(\%)^{e}\end{array}$ & $\begin{array}{c}T_{\mathrm{m}} \\
\left({ }^{\circ} \mathrm{C}\right)^{f} \\
\end{array}$ \\
\hline 1 & $\boldsymbol{M}-\mathrm{N}_{3}-\mathrm{PLLA}^{0.9 \mathrm{~K}}$ & $\boldsymbol{M}-\mathbf{L}_{\mathrm{N} 3}-\mathbf{1}^{1.0 \mathrm{~K}_{-} \mathrm{I}}$ & 64 & 1.61 & 1.19 & 0.95 & 6.6 & $>99$ & 96 \\
\hline 2 & $\boldsymbol{M}-\mathrm{N}_{3}-\mathrm{PLLA}^{2.1 \mathrm{~K}}$ & $\boldsymbol{M}-\mathbf{L}_{\mathrm{N} 3}-\mathbf{1}^{2.3 \mathrm{~K}_{-} \mathrm{I}}$ & 69 & 4.14 & 1.11 & 2.30 & 16 & $>99$ & 137 \\
\hline 3 & $\boldsymbol{M}-\mathrm{N}_{3}-\mathrm{PLLA}^{3.9 \mathrm{~K}}$ & $\boldsymbol{M}-\mathbf{L}_{\mathrm{N} 3}-\mathbf{1}^{4.0 \mathrm{~K}_{-\mathrm{I}}}$ & 82 & 6.53 & 1.12 & 4.01 & 28 & $>99$ & 149 \\
\hline 4 & $\boldsymbol{P}-\mathrm{N}_{3}-\mathrm{PDLA}^{0.9 \mathrm{~K}}$ & $\boldsymbol{P}-\mathbf{D}_{\mathrm{N} 3}-\mathbf{1}^{1.0 \mathrm{~K}_{-}}-\mathrm{F}$ & 72 & 1.62 & 1.23 & 0.98 & 6.8 & $>99$ & 95 \\
\hline 5 & $\boldsymbol{P}-\mathrm{N}_{3}-\mathrm{PDLA}^{2.3 \mathrm{~K}}$ & $\boldsymbol{P}-\mathbf{D}_{\mathrm{N} 3}-\mathbf{1}^{2.4 \mathrm{~K}_{-}}-\mathrm{F}$ & 93 & 4.15 & 1.11 & 2.41 & 17 & $>99$ & 138 \\
\hline 6 & $\boldsymbol{P}-\mathrm{N}_{3}-\mathrm{PDLA}^{4.0 \mathrm{~K}}$ & $\boldsymbol{P}-\mathbf{D}_{\mathrm{N} 3}-\mathbf{1}^{4.1 \mathrm{~K}_{-}}-\mathrm{F}$ & 98 & 7.38 & 1.12 & 4.11 & 29 & $>99$ & 149 \\
\hline
\end{tabular}
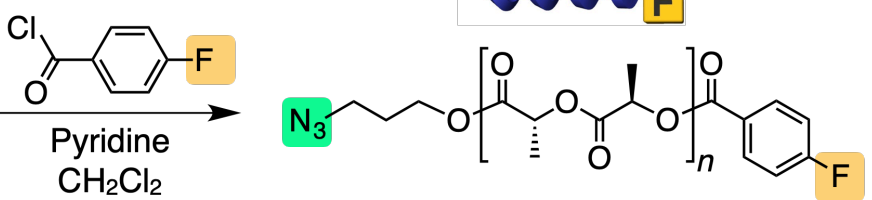

$\mathrm{DP}_{\mathrm{n}}=6.8: P-\mathrm{D}_{\mathrm{N} 3}-11^{1.0 \mathrm{~K}}-\mathrm{F}$ (run 4)

$\mathrm{DP}_{\mathrm{n}}=17: P-\mathrm{D}_{\mathrm{N} 3}-\mathbf{1}^{2.4 \mathrm{~K}}-\mathrm{F}$ (run 5)

$\mathrm{DP}_{\mathrm{n}}=29: P-\mathrm{D}_{\mathrm{N} 3}-\mathbf{1}^{4.1 \mathrm{~K}}-\mathrm{F}$ (run 6)

${ }^{a}$ Isolated yield after the purification by recycle SEC. ${ }^{b}$ Estimated by SEC (polystyrene standards) with $\mathrm{CHCl}_{3}$ as the eluent. ${ }^{c}$ Determined by the ${ }^{1} \mathrm{H}$ NMR end-group analysis in $\mathrm{CDCl}_{3}{ }^{d}$ Number-average degree of polymerization determined by $M_{\mathrm{n}, \mathrm{NMR}}{ }^{e}$ Functionality of the end-capped benzoate residue estimated by ${ }^{1} \mathrm{H} \mathrm{NMR}$ in $\mathrm{CDCl}_{3} .{ }^{f}$ Melting point determined by DSC.

Spectroscopic data of $\boldsymbol{M}-\mathbf{L}_{\mathrm{N} 3} \mathbf{1}^{1.0 \mathrm{~K}-\mathrm{I}: \mathrm{IR}}\left(\mathrm{KBr}, \mathrm{cm}^{-1}\right): 2101(\mathrm{~N}=\mathrm{N}=\mathrm{N}), 1759(\mathrm{C}=\mathrm{O}), 1188(\mathrm{C}-\mathrm{O}), 1094$ (C-O). ${ }^{1} \mathrm{H}$ NMR $\left(500 \mathrm{MHz}, \mathrm{CDCl}_{3}, 25^{\circ} \mathrm{C}\right): \delta 7.82-7.77(\mathrm{~m}, \mathrm{Ar}-\mathrm{H}, 4 \mathrm{H}), 5.34(\mathrm{q}, J=7.0 \mathrm{~Hz}, \mathrm{CH}$ PLA backbone, 1H), 5.22-5.11 (m, CH PLA backbone, 12H), 4.24 (dt, $\left.J=6.1,2.5 \mathrm{~Hz}, \mathrm{OCH}_{2}, 2 \mathrm{H}\right), 3.39$ (t, $\left.J=6.6 \mathrm{~Hz}, \mathrm{~N}_{3} \mathrm{CH}_{2}, 2 \mathrm{H}\right), 1.94-1.89$ (m, $\left.\mathrm{OCH}_{2} \mathrm{CH}_{2}, 2 \mathrm{H}\right), 1.70-1.51$ (m, $\mathrm{CH}_{3}$ PLA backbone, partially overlapping with $\mathrm{H}_{2} \mathrm{O}$ signal) (for all the peak assignments including the end groups, see Figure S2a(ii)).

Spectroscopic data of $\boldsymbol{M}-\mathbf{L}_{\mathrm{N} 3}-\mathbf{1}^{2.3 \mathrm{~K}}-\mathrm{I}$ : IR $\left(\mathrm{KBr}, \mathrm{cm}^{-1}\right): 2103(\mathrm{~N}=\mathrm{N}=\mathrm{N}), 1759(\mathrm{C}=\mathrm{O}), 1185(\mathrm{C}-\mathrm{O}), 1092$ (C-O). ${ }^{1} \mathrm{H}$ NMR $\left(500 \mathrm{MHz}, \mathrm{CDCl}_{3}, 25^{\circ} \mathrm{C}\right): \delta 7.82-7.77(\mathrm{~m}, \mathrm{Ar}-\mathrm{H}, 4 \mathrm{H}), 5.35(\mathrm{q}, J=7.1 \mathrm{~Hz}, \mathrm{CH}$ PLA 
backbone, 1H), 5.23-5.10 (m, CH PLA backbone, 31H), 4.24 (dt, $\left.J=6.0,2.5 \mathrm{~Hz}, \mathrm{OCH}_{2}, 2 \mathrm{H}\right), 3.39$ (t, $\left.J=6.7 \mathrm{~Hz}, \mathrm{~N}_{3} \mathrm{CH}_{2}, 2 \mathrm{H}\right), 1.94-1.89$ (m, $\left.\mathrm{OCH}_{2} \mathrm{CH}_{2}, 2 \mathrm{H}\right), 1.72-1.44$ (m, $\mathrm{CH}_{3}$ PLA backbone, partially overlapping with $\mathrm{H}_{2} \mathrm{O}$ signal) (for all the peak assignments including the end groups, see Figure S3a(ii)).

Spectroscopic data of $\boldsymbol{M}-\mathrm{L}_{\mathrm{N} 3} \mathbf{1}^{4.0 \mathrm{~K}}-\mathrm{I}$ : IR $\left(\mathrm{KBr}, \mathrm{cm}^{-1}\right): 2103(\mathrm{~N}=\mathrm{N}=\mathrm{N}), 1759(\mathrm{C}=\mathrm{O}), 1184(\mathrm{C}-\mathrm{O}), 1090$ (C-O). ${ }^{1} \mathrm{H}$ NMR $\left(500 \mathrm{MHz}, \mathrm{CDCl}_{3}, 25^{\circ} \mathrm{C}\right): \delta 7.82-7.77(\mathrm{~m}, \mathrm{Ar}-\mathrm{H}, 4 \mathrm{H}), 5.34(\mathrm{q}, J=7.1 \mathrm{~Hz}, \mathrm{CH}$ PLA backbone, 1H), 5.22-5.11 (m, CH PLA backbone, 55H), 4.24 (dt, $\left.J=6.0,2.5 \mathrm{~Hz}, \mathrm{OCH}_{2}, 2 \mathrm{H}\right), 3.39$ (t, $\left.J=6.7 \mathrm{~Hz}, \mathrm{~N}_{3} \mathrm{CH}_{2}, 2 \mathrm{H}\right), 1.94-1.89$ (m, $\left.\mathrm{OCH}_{2} \mathrm{CH}_{2}, 2 \mathrm{H}\right), 1.72-1.52$ (m, $\mathrm{CH}_{3}$ PLA backbone, partially overlapping with $\mathrm{H}_{2} \mathrm{O}$ signal) (for all the peak assignments including the end groups, see Figure 2a(ii)).

Spectroscopic data of $\boldsymbol{P}-\mathbf{D}_{\mathrm{N} 3}-\mathbf{1}^{1.0 \mathrm{~K}}-\mathrm{F}$ : IR $\left(\mathrm{KBr}, \mathrm{cm}^{-1}\right): 2103(\mathrm{~N}=\mathrm{N}=\mathrm{N}), 1759(\mathrm{C}=\mathrm{O}), 1186(\mathrm{C}-\mathrm{O}), 1092$ (C-O). ${ }^{1} \mathrm{H}$ NMR $\left(500 \mathrm{MHz}, \mathrm{CDCl}_{3}, 25^{\circ} \mathrm{C}\right): \delta 8.11-8.08(\mathrm{~m}, \mathrm{Ar}-\mathrm{H}, 2 \mathrm{H}), 7.13-7.10(\mathrm{~m}, \mathrm{Ar}-\mathrm{H}, 2 \mathrm{H})$, 5.35 (q, $J=7.1 \mathrm{~Hz}$, CH PLA backbone, 1H), 5.24-5.10 (m, CH PLA backbone, 13H), 4.24 (dt, $J=$ 6.0, $\left.2.5 \mathrm{~Hz}, \mathrm{OCH}_{2}, 2 \mathrm{H}\right), 3.38$ (t, $\left.J=6.7 \mathrm{~Hz}, \mathrm{~N}_{3} \mathrm{CH}_{2}, 2 \mathrm{H}\right), 1.94-1.89$ (m, $\left.\mathrm{OCH}_{2} \mathrm{CH}_{2}, 2 \mathrm{H}\right), 1.71-1.51$ (m, $\mathrm{CH}_{3}$ PLA backbone, partially overlapping with $\mathrm{H}_{2} \mathrm{O}$ signal) (for all the peak assignments including the end groups, see Figure S4a(ii)).

Spectroscopic data of $\boldsymbol{P}-\mathbf{D}_{\mathrm{N} 3}-\mathbf{1}^{2.4 \mathrm{~K}}-\mathrm{F}$ : IR $\left(\mathrm{KBr}, \mathrm{cm}^{-1}\right): 2103(\mathrm{~N}=\mathrm{N}=\mathrm{N}), 1759(\mathrm{C}=\mathrm{O}), 1185(\mathrm{C}-\mathrm{O}), 1092$ (C-O). ${ }^{1} \mathrm{H}$ NMR (500 MHz, $\left.\mathrm{CDCl}_{3}, 25{ }^{\circ} \mathrm{C}\right): \delta 8.11-8.08(\mathrm{~m}, \mathrm{Ar}-\mathrm{H}, 2 \mathrm{H}), 7.13-7.10$ (m, Ar-H, 2H), 5.35 (q, $J=7.1 \mathrm{~Hz}$, CH PLA backbone, 1H), 5.24-5.10 (m, CH PLA backbone, 32H), 4.24 (dt, $J=$ 6.0, $\left.2.5 \mathrm{~Hz}, \mathrm{OCH}_{2}, 2 \mathrm{H}\right), 3.38$ ( $\left.\mathrm{t}, J=6.7 \mathrm{~Hz}, \mathrm{~N}_{3} \mathrm{CH}_{2}, 2 \mathrm{H}\right), 1.94-1.89\left(\mathrm{~m}, \mathrm{OCH}_{2} \mathrm{CH}_{2}, 2 \mathrm{H}\right), 1.72-1.51$ (m, $\mathrm{CH}_{3}$ PLA backbone, partially overlapping with $\mathrm{H}_{2} \mathrm{O}$ signal) (for all the peak assignments including the end groups, see Figure S5a(ii)).

Spectroscopic data of $\boldsymbol{P}-\mathbf{D}_{\mathrm{N} 3}-\mathbf{1}^{4.1 \mathrm{~K}}-\mathrm{F}$ : IR $\left(\mathrm{KBr}, \mathrm{cm}^{-1}\right): 2103(\mathrm{~N}=\mathrm{N}=\mathrm{N}), 1759(\mathrm{C}=\mathrm{O}), 1184(\mathrm{C}-\mathrm{O})$, $1090(\mathrm{C}-\mathrm{O}) .{ }^{1} \mathrm{H}$ NMR $\left(500 \mathrm{MHz}, \mathrm{CDCl}_{3}, 25^{\circ} \mathrm{C}\right): \delta 8.11-8.08(\mathrm{~m}, \mathrm{Ar}-\mathrm{H}, 2 \mathrm{H}), 7.14-7.10(\mathrm{~m}, \mathrm{Ar}-\mathrm{H}$, 2H), 5.35 (q, $J=7.1 \mathrm{~Hz}, \mathrm{CH}$ PLA backbone, 1H), 5.22-5.11 (m, CH PLA backbone, 56H), 4.24 (dt, $\left.J=6.0,2.5 \mathrm{~Hz}, \mathrm{OCH}_{2}, 2 \mathrm{H}\right), 3.39\left(\mathrm{t}, J=6.7 \mathrm{~Hz}, \mathrm{~N}_{3} \mathrm{CH}_{2}, 2 \mathrm{H}\right), 1.94-1.89\left(\mathrm{~m}, \mathrm{OCH}_{2} \mathrm{CH}_{2}, 2 \mathrm{H}\right), 1.72-$ 1.44 (m, $\mathrm{CH}_{3}$ PLA backbone, partially overlapping with $\mathrm{H}_{2} \mathrm{O}$ signal) (for all the peak assignments including the end groups, see Figure S6a(ii)). 


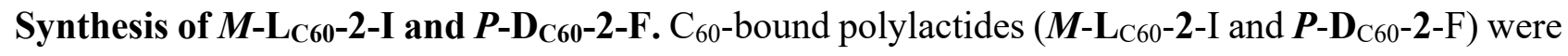
prepared by the copper(I)-catalyzed click reaction of $\mathrm{N}_{3}-1$ PLAs with $\mathrm{E}_{-} \mathrm{C}_{60}$.

A typical procedure for the copper(I)-catalyzed click reaction of $\boldsymbol{P}-\mathbf{D}_{\mathrm{N} 3}-\mathbf{1}^{1.0 \mathrm{~K}}-\mathrm{F}$ with $\mathrm{E}-\mathrm{C}_{60}$ is described as follows. To a mixture of $\boldsymbol{P}-\mathbf{D}_{\mathrm{N} 3}-\mathbf{1}^{1.0 \mathrm{~K}}-\mathrm{F}(70 \mathrm{mg}, 58 \mu \mathrm{mol})$ and E-C $60(59 \mathrm{mg}, 69 \mu \mathrm{mol})$ in anhydrous dichloromethane/o-DCB (4 mL, 1/1, v/v) was added $N, N, N^{\prime}, N^{\prime \prime}, N^{\prime \prime}-$ pentamethyldiethylenetriamine (PMDETA) $(15 \mu \mathrm{L}, 72 \mu \mathrm{mol})$ under nitrogen, and the solution was degassed with three freeze-pump-thaw cycles. To this was added $\mathrm{CuBr}(12 \mathrm{mg}, 84 \mu \mathrm{mol})$ under nitrogen and the reaction mixture was degassed again, and stirred at room temperature for $16 \mathrm{~h}$. After removal of dichloromethane under reduced pressure, to this was added methanol $(2 \mathrm{~mL})$ to precipitate the desired product. The precipitate was collected by centrifugation and purified by SEC on Bio Beads $\mathrm{S}-\mathrm{X} 1 \mathrm{using}$ dichloromethane as the eluent and preparative recycling SEC (JAIGEL-1H and -2H columns, chloroform) to give $\boldsymbol{P}-\mathbf{D}_{\mathrm{C} 60-2^{1.0 K}-\mathrm{F}}(98 \mathrm{mg}, 82 \%$ yield) as a brown solid. In the same way, $\boldsymbol{P}$-D $\mathbf{D}_{\mathrm{C} 60-2}-\mathrm{F}$ and $\boldsymbol{M}$-L $\mathbf{L}_{\mathrm{C} 60-2-I}$ with different molar masses were prepared. The $M_{\mathrm{n}}$ and $M_{\mathrm{w}} / M_{\mathrm{n}}$ of the polymers were determined by SEC using polystyrene standards in chloroform. The $M_{\mathrm{n}}$ values were also estimated by ${ }^{1} \mathrm{H}$ NMR end-group analysis $\left(M_{\mathrm{n}, \mathrm{NMR}}\right)$ in $\mathrm{CDCl}_{3}$. The copper(I)-catalyzed click reaction results are summarized in Table S3. 

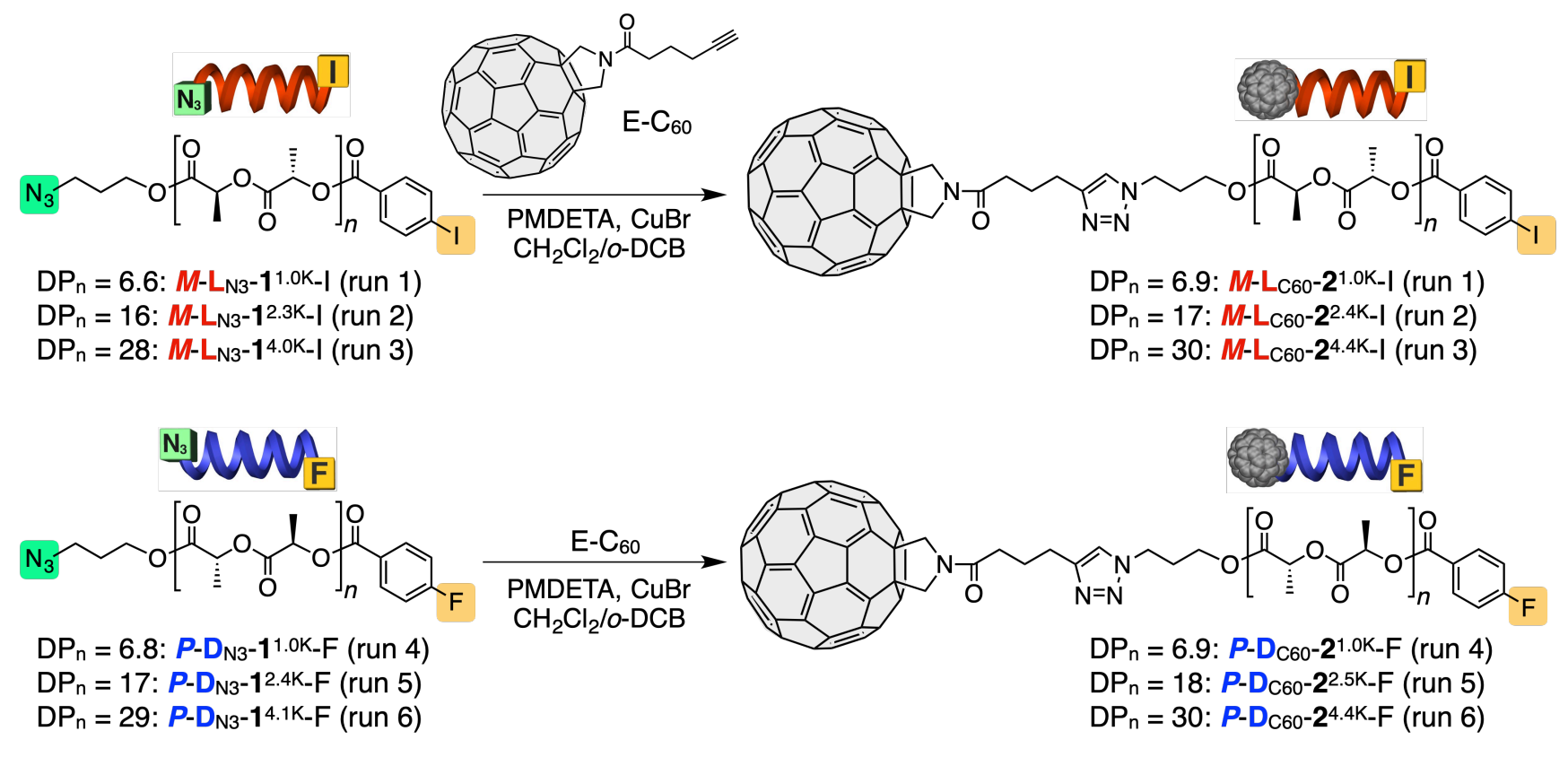

\begin{tabular}{|c|c|c|c|c|c|c|c|c|c|}
\hline run & reactant & product & $\begin{array}{l}\text { yield } \\
(\%)^{a}\end{array}$ & $\begin{array}{l}M_{\mathrm{n}, \mathrm{SEC}} \\
\left(10^{3}\right)^{b}\end{array}$ & $M_{\mathrm{w}} / M_{\mathrm{n}}{ }^{b}$ & $\begin{array}{c}M_{\mathrm{n}, \mathrm{NMR}} \\
\left(10^{3}\right)^{c}\end{array}$ & $\mathrm{DP}_{\mathrm{n}, \mathrm{NMR}}{ }^{d}$ & $\begin{array}{l}f_{\mathrm{C} 60} \\
(\%)^{e}\end{array}$ & $\begin{array}{c}T_{\mathrm{m}} \\
\left({ }^{\circ} \mathrm{C}\right)^{f}\end{array}$ \\
\hline 1 & $\boldsymbol{M}-\mathbf{L}_{\mathrm{N} 3}-\mathbf{1}^{1.0 \mathrm{~K}_{-} \mathrm{I}}$ & $M-\mathbf{L}_{\mathrm{C} 60-2^{1.0 \mathrm{~K}_{-I}}}$ & 88 & 1.82 & 1.24 & 0.99 & 6.9 & $>99$ & $-g$ \\
\hline 2 & $\boldsymbol{M}-\mathbf{L}_{\mathrm{N} 3}-\mathbf{1}^{2.3 \mathrm{~K}_{-} \mathrm{I}}$ & $\boldsymbol{M}-\mathbf{L}_{\mathrm{C} 60}-\mathbf{2}^{2.4 \mathrm{~K}_{-} \mathrm{I}}$ & 79 & 4.38 & 1.20 & 2.43 & 17 & $>99$ & 141 \\
\hline 3 & $\boldsymbol{M}-\mathbf{L}_{\mathrm{N} 3}-\mathbf{1}^{4.0 \mathrm{~K}_{-} \mathrm{I}}$ & $M-\mathbf{L}_{\mathrm{C} 60}-2^{4.4 \mathrm{~K}_{-I}}$ & 73 & 7.73 & 1.32 & 4.36 & 30 & $>99$ & 146 \\
\hline 4 & $\boldsymbol{P}-\mathbf{D}_{\mathrm{N} 3}-\mathbf{1}^{1.0 \mathrm{~K}_{-} \mathrm{F}}$ & $\boldsymbol{P}-\mathbf{D}_{\mathrm{C} 60-} \mathbf{2}^{1.0 \mathrm{~K}_{-}}-\mathrm{F}$ & 82 & 1.63 & 1.41 & 1.00 & 6.9 & $>99$ & $-g$ \\
\hline 5 & $\boldsymbol{P}-\mathbf{D}_{\mathrm{N} 3}-\mathbf{1}^{2.4 \mathrm{~K}_{-}}-\mathrm{F}$ & 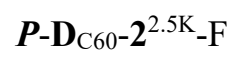 & 94 & 4.75 & 1.26 & 2.52 & 18 & $>99$ & 141 \\
\hline 6 & $\boldsymbol{P}-\mathbf{D}_{\mathrm{N} 3}-\mathbf{1}^{4.1 \mathrm{~K}_{-} \mathrm{F}}$ & $\boldsymbol{P}-\mathbf{D}_{\mathrm{C} 60-\mathbf{2}^{4.4 \mathrm{~K}_{-}}-\mathrm{F}}$ & 87 & 7.93 & 1.35 & 4.39 & 30 & $>99$ & 146 \\
\hline
\end{tabular}

${ }^{a}$ Isolated yield after the purification by recycle SEC. ${ }^{b}$ Estimated by SEC (polystyrene standards) with $\mathrm{CHCl}_{3}$ as the eluent. ${ }^{c}$ Determined by the ${ }^{1} \mathrm{H}$ NMR end-group analysis in $\mathrm{CDCl}_{3}$. ${ }^{d}$ Number-average degree of polymerization determined by $M_{\mathrm{n}, \mathrm{NMR}} \cdot{ }^{e}$ Functionality of the end-capped $\mathrm{C}_{60}$ residue estimated by ${ }^{1} \mathrm{H} \mathrm{NMR}$ in $\mathrm{CDCl}_{3 .}{ }^{f}$ Melting point determined by DSC. ${ }^{g}$ Not observed in the DSC thermogram.

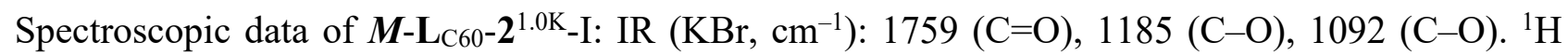
NMR (500 MHz, $\left.\mathrm{CDCl}_{3}, 25^{\circ} \mathrm{C}\right): \delta 7.82-7.77(\mathrm{~m}, \mathrm{Ar}-\mathrm{H}, 4 \mathrm{H}), 7.46(\mathrm{~s}, \mathrm{C}=\mathrm{CH}, 1 \mathrm{H}), 5.48\left(\mathrm{~s}, \mathrm{C}_{60}-\mathrm{CH}_{2} \mathrm{~N}\right.$, 2H), $5.40\left(\mathrm{~s}, \mathrm{C}_{60}-\mathrm{CH}_{2} \mathrm{~N}, 2 \mathrm{H}\right), 5.35$ (q, $J=7.1 \mathrm{~Hz}, \mathrm{CH}$ PLA backbone, 1H), 5.23-5.08 (m, CH PLA backbone, 13H), $4.42\left(\mathrm{dt}, J=6.7,3.1 \mathrm{~Hz}, \mathrm{OCH}_{2}, 2 \mathrm{H}\right), 4.23-4.11\left(\mathrm{~m}, \mathrm{NCH}_{2}, 2 \mathrm{H}\right), 2.99$ (t, $J=7.3 \mathrm{~Hz}$, $\left.\mathrm{CH}_{2}, 2 \mathrm{H}\right), 2.92\left(\mathrm{t}, J=7.3 \mathrm{~Hz}, \mathrm{CH}_{2}, 2 \mathrm{H}\right), 2.35-2.28\left(\mathrm{~m}, \mathrm{OCH}_{2} \mathrm{CH}_{2}, 4 \mathrm{H}\right), 1.70-1.53$ (m, $\mathrm{CH}_{3}$ PLA backbone, partially overlapping with $\mathrm{H}_{2} \mathrm{O}$ signal) (for all the peak assignments including the end groups, see Figure S2a(iii)). 


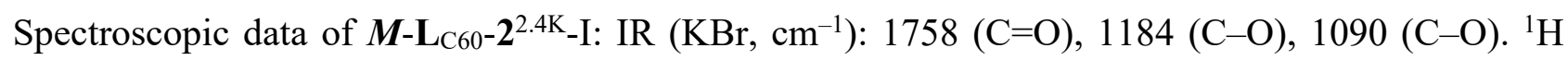
NMR (500 MHz, $\left.\mathrm{CDCl}_{3}, 25^{\circ} \mathrm{C}\right): \delta 7.82-7.77(\mathrm{~m}, \mathrm{Ar}-\mathrm{H}, 4 \mathrm{H}), 7.45(\mathrm{~s}, \mathrm{C}=\mathrm{CH}, 1 \mathrm{H}), 5.48\left(\mathrm{~s}, \mathrm{C}_{60}-\mathrm{CH}_{2} \mathrm{~N}\right.$, 2H), $5.40\left(\mathrm{~s}, \mathrm{C}_{60}-\mathrm{CH}_{2} \mathrm{~N}, 2 \mathrm{H}\right), 5.34$ (q, $J=7.1 \mathrm{~Hz}, \mathrm{CH}$ PLA backbone, 1H), 5.23-5.08 (m, CH PLA backbone, 33H), $4.42\left(\mathrm{dt}, J=6.7,3.1 \mathrm{~Hz}, \mathrm{OCH}_{2}, 2 \mathrm{H}\right), 4.24-4.11\left(\mathrm{~m}, \mathrm{NCH}_{2}, 2 \mathrm{H}\right), 2.98(\mathrm{t}, J=7.3 \mathrm{~Hz}$, $\left.\mathrm{CH}_{2}, 2 \mathrm{H}\right), 2.92\left(\mathrm{t}, J=7.4 \mathrm{~Hz}, \mathrm{CH}_{2}, 2 \mathrm{H}\right), 2.35-2.28\left(\mathrm{~m}, \mathrm{OCH}_{2} \mathrm{CH}_{2}, 4 \mathrm{H}\right), 1.72-1.53\left(\mathrm{~m}, \mathrm{CH}_{3}\right.$ PLA backbone, partially overlapping with $\mathrm{H}_{2} \mathrm{O}$ signal) (for all the peak assignments including the end groups, see Figure S3a(iii)).

Spectroscopic data of $\boldsymbol{M}$-L $\mathbf{L}_{\mathrm{C} 60-\mathbf{2}^{4} \cdot 4 \mathrm{~K}} \mathrm{I}$ : IR $\left(\mathrm{KBr}, \mathrm{cm}^{-1}\right): 1758(\mathrm{C}=\mathrm{O}), 1184(\mathrm{C}-\mathrm{O}), 1090(\mathrm{C}-\mathrm{O}) .{ }^{1} \mathrm{H}$ $\operatorname{NMR}\left(500 \mathrm{MHz}, \mathrm{CDCl}_{3}, 25^{\circ} \mathrm{C}\right): \delta 7.82-7.77(\mathrm{~m}, \mathrm{Ar}-\mathrm{H}, 4 \mathrm{H}), 7.45(\mathrm{~s}, \mathrm{C}=\mathrm{CH}, 1 \mathrm{H}), 5.48\left(\mathrm{~s}, \mathrm{C}_{60}-\mathrm{CH}_{2} \mathrm{~N}\right.$, 2H), $5.40\left(\mathrm{~s}, \mathrm{C}_{60}-\mathrm{CH}_{2} \mathrm{~N}, 2 \mathrm{H}\right), 5.35$ (q, $J=7.0 \mathrm{~Hz}, \mathrm{CH}$ PLA backbone, 1H), 5.22-5.09 (m, CH PLA backbone, $59 \mathrm{H}), 4.42\left(\mathrm{dt}, J=6.5,3.0 \mathrm{~Hz}, \mathrm{OCH}_{2}, 2 \mathrm{H}\right), 4.23-4.11\left(\mathrm{~m}, \mathrm{NCH}_{2}, 2 \mathrm{H}\right), 2.98(\mathrm{t}, J=7.4 \mathrm{~Hz}$, $\left.\mathrm{CH}_{2}, 2 \mathrm{H}\right), 2.92\left(\mathrm{t}, J=7.4 \mathrm{~Hz}, \mathrm{CH}_{2}, 2 \mathrm{H}\right), 2.34-2.28\left(\mathrm{~m}, \mathrm{OCH}_{2} \mathrm{CH}_{2}, 4 \mathrm{H}\right), 1.72-1.44\left(\mathrm{~m}, \mathrm{CH}_{3}\right.$ PLA backbone, partially overlapping with $\mathrm{H}_{2} \mathrm{O}$ signal) (for all the peak assignments including the end groups, see Figure 2a(iii)).

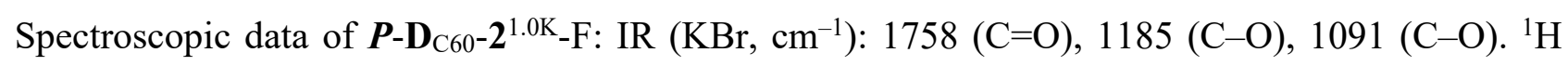
NMR (500 MHz, $\left.\mathrm{CDCl}_{3}, 25^{\circ} \mathrm{C}\right): \delta 8.12-8.08(\mathrm{~m}, \mathrm{Ar}-\mathrm{H}, 2 \mathrm{H}), 7.45(\mathrm{~s}, \mathrm{C}=\mathrm{CH}, 1 \mathrm{H}), 7.14-7.09(\mathrm{~m}, \mathrm{Ar}-$ $\mathrm{H}, 2 \mathrm{H}), 5.48\left(\mathrm{~s}, \mathrm{C}_{60}-\mathrm{CH}_{2} \mathrm{~N}, 2 \mathrm{H}\right), 5.39\left(\mathrm{~s}, \mathrm{C}_{60}-\mathrm{CH}_{2} \mathrm{~N}, 2 \mathrm{H}\right), 5.35$ (q, $J=7.0 \mathrm{~Hz}, \mathrm{CH}$ PLA backbone, 1H), 5.24-5.08 (m, CH PLA backbone, 13H), 4.42 (dt, $\left.J=6.7,3.1 \mathrm{~Hz}, \mathrm{OCH}_{2}, 2 \mathrm{H}\right), 4.23-4.11$ (m, $\left.\mathrm{NCH}_{2}, 2 \mathrm{H}\right), 2.98$ (t, $\left.J=7.3 \mathrm{~Hz}, \mathrm{CH}_{2}, 2 \mathrm{H}\right), 2.92$ (t, $\left.J=7.3 \mathrm{~Hz}, \mathrm{CH}_{2}, 2 \mathrm{H}\right), 2.36\left(\mathrm{~m}, \mathrm{OCH}_{2} \mathrm{CH}_{2}, 4 \mathrm{H}\right)$, 1.70-1.53 (m, $\mathrm{CH}_{3}$ PLA backbone, partially overlapping with $\mathrm{H}_{2} \mathrm{O}$ signal) (for all the peak assignments including the end groups, see Figure S4a(iii)).

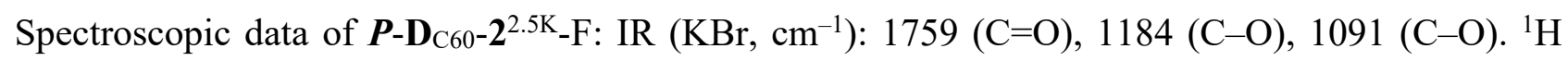
NMR (500 MHz, $\left.\mathrm{CDCl}_{3}, 25^{\circ} \mathrm{C}\right): \delta 8.12-8.08(\mathrm{~m}, \mathrm{Ar}-\mathrm{H}, 2 \mathrm{H}), 7.45(\mathrm{~s}, \mathrm{C}=\mathrm{CH}, 1 \mathrm{H}), 7.14-7.09(\mathrm{~m}, \mathrm{Ar}-$ $\mathrm{H}, 2 \mathrm{H}), 5.48\left(\mathrm{~s}, \mathrm{C}_{60}-\mathrm{CH}_{2} \mathrm{~N}, 2 \mathrm{H}\right), 5.40\left(\mathrm{~s}, \mathrm{C}_{60}-\mathrm{CH}_{2} \mathrm{~N}, 2 \mathrm{H}\right), 5.35$ (q, $J=7.1 \mathrm{~Hz}, \mathrm{CH}$ PLA backbone, 1H), 5.22-5.09 (m, CH PLA backbone, 34H), 4.42 (dt, $\left.J=6.7,3.1 \mathrm{~Hz}, \mathrm{OCH}_{2}, 2 \mathrm{H}\right), 4.23-4.11$ (m, $\left.\mathrm{NCH}_{2}, 2 \mathrm{H}\right), 2.98$ (t, $\left.J=7.4 \mathrm{~Hz}, \mathrm{CH}_{2}, 2 \mathrm{H}\right), 2.92\left(\mathrm{t}, J=7.4 \mathrm{~Hz}, \mathrm{CH}_{2}, 2 \mathrm{H}\right), 2.36-2.26\left(\mathrm{~m}, \mathrm{OCH}_{2} \mathrm{CH}_{2}\right.$, $4 \mathrm{H}), 1.71-1.53$ (m, $\mathrm{CH}_{3}$ PLA backbone, partially overlapping with $\mathrm{H}_{2} \mathrm{O}$ signal) (for all the peak assignments including the end groups, see Figure S5a(iii)).

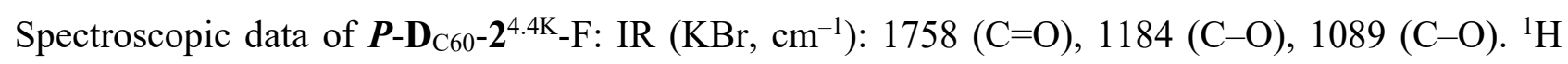
NMR (500 MHz, $\left.\mathrm{CDCl}_{3}, 25^{\circ} \mathrm{C}\right): \delta 8.11-8.08(\mathrm{~m}, \mathrm{Ar}-\mathrm{H}, 2 \mathrm{H}), 7.45(\mathrm{~s}, \mathrm{C}=\mathrm{CH}, 1 \mathrm{H}), 7.14-7.09(\mathrm{~m}, \mathrm{Ar}-$ $\mathrm{H}, 2 \mathrm{H}), 5.48\left(\mathrm{~s}, \mathrm{C}_{60}-\mathrm{CH}_{2} \mathrm{~N}, 2 \mathrm{H}\right), 5.40\left(\mathrm{~s}, \mathrm{C}_{60}-\mathrm{CH}_{2} \mathrm{~N}, 2 \mathrm{H}\right), 5.35$ (q, J=7.1 Hz, CH PLA backbone, 
1H), 5.22-5.08 (m, CH PLA backbone, 60H), 4.42 (dt, $\left.J=6.7,3.1 \mathrm{~Hz}, \mathrm{OCH}_{2}, 2 \mathrm{H}\right), 4.23-4.11$ (m, $\left.\mathrm{NCH}_{2}, 2 \mathrm{H}\right), 2.98$ (t, $\left.J=7.4 \mathrm{~Hz}, \mathrm{CH}_{2}, 2 \mathrm{H}\right), 2.92\left(\mathrm{t}, J=7.4 \mathrm{~Hz}, \mathrm{CH}_{2}, 2 \mathrm{H}\right), 2.35-2.28\left(\mathrm{~m}, \mathrm{OCH}_{2} \mathrm{CH}_{2}\right.$, $4 \mathrm{H}), 1.72-1.54$ (m, $\mathrm{CH}_{3}$ PLA backbone, partially overlapping with $\mathrm{H}_{2} \mathrm{O}$ signal) (for all the peak assignments including the end groups, see Figure S6a(iii)).

\section{Preparation of st-PMMA Films Complexed with the $\mathrm{C}_{60}$-Free and $\mathrm{C}_{60}$-Bound $M$ - and $P$-PLAs}

st-PMMA/PLA complex films for the DSC and XRD measurements were prepared according to the method reported previously for the preparation of st-PMMA/C 60 -peptide complex films. ${ }^{\mathrm{S} 4}$

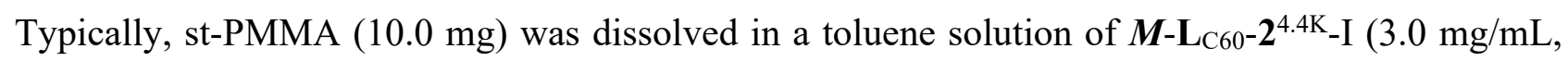
$1.0 \mathrm{~mL}$ ) at $110^{\circ} \mathrm{C}$. After the solution was cooled to room temperature, the solution gelled within 10 min. The resulting soft gel was centrifuged at $1700 \mathrm{~g}$ for $10 \mathrm{~min}$ and the supernatant containing the unencapsulated $\boldsymbol{M}-\mathbf{L}_{\mathrm{C} 60}-\mathbf{2}^{4.4 \mathrm{~K}_{-}}$I was removed from the gel by decantation. The condensed gel was washed with toluene and the solvent was removed by decantation after centrifugation. This procedure

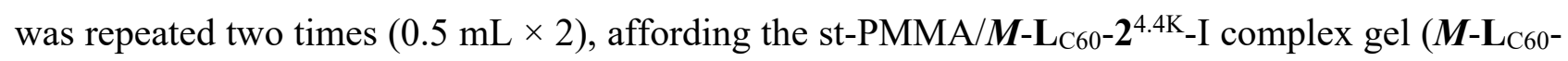

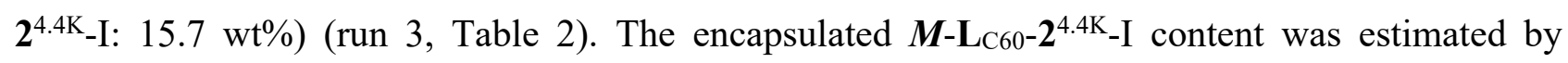
absorption spectroscopy using the following equation: the encapsulated $\boldsymbol{M}$ - $\mathbf{L}_{\mathrm{C} 60}-\mathbf{2}^{4.4 \mathrm{~K}}-\mathrm{I}$ content $(\mathrm{mg})$

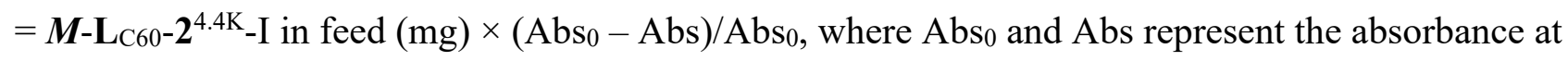
$313 \mathrm{~nm}$ of the feed $\boldsymbol{M}-\mathbf{L}_{\mathrm{C} 60}-\mathbf{2}^{4.4 \mathrm{~K}_{-} \mathrm{I}}$ solution and that of the supernatant separated from the st-

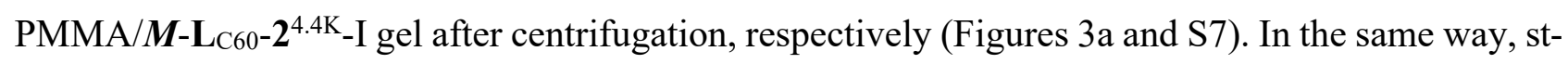

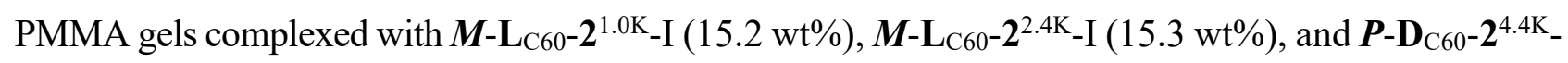

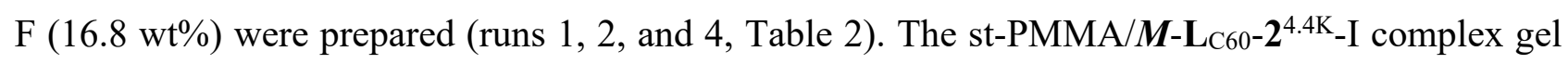

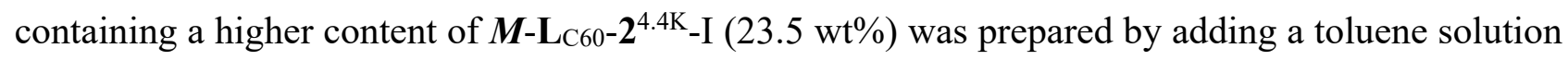
$(0.4 \mathrm{~mL})$ of $\boldsymbol{M}-\mathbf{L}_{\mathrm{C} 60}-\mathbf{2}^{4.4 \mathrm{~K}_{-} \mathrm{I}}(9.0 \mathrm{mg} / \mathrm{mL})$ to st-PMMA $(4.0 \mathrm{mg})$ followed by heating to $110^{\circ} \mathrm{C}$ and cooling to room temperature, and then centrifuged and washed with toluene (run 5, Table 2). The stPMMA/M-L $\mathbf{L}_{\mathrm{N} 3}-\mathbf{1}^{4.0 \mathrm{~K}_{-}}$c complex gel $\left(\boldsymbol{M}-\mathbf{L}_{\mathrm{N} 3}-\mathbf{1}^{4.0 \mathrm{~K}_{-}}\right.$: $\left.12.6 \mathrm{wt} \%\right)$ was also prepared by adding a toluene$d_{8}$ solution $(0.8 \mathrm{~mL})$ of $\boldsymbol{M}-\mathbf{L}_{\mathrm{N} 3}-\mathbf{1}^{4.0 \mathrm{~K}_{-} \mathrm{I}}(14.5 \mathrm{mg} / \mathrm{mL})$ to st-PMMA $(8.0 \mathrm{mg})$ followed by heating to $110^{\circ} \mathrm{C}$ and cooling to room temperature, and then centrifuged and washed with toluene (run 6 , Table

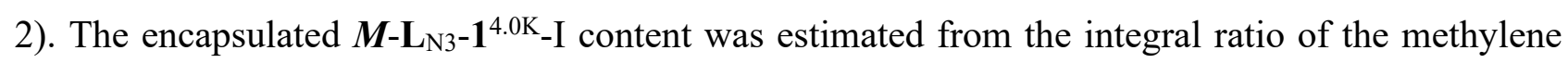
protons of the $\mathrm{N}_{3}-1^{4.0 \mathrm{~K}}$ end-group relative to the ${ }^{13} \mathrm{C}$ satellite peaks of the methoxy protons of stPMMA due to ${ }^{13} \mathrm{C}-{ }^{1} \mathrm{H}$ coupling using ${ }^{1} \mathrm{H}$ NMR spectroscopy ${ }^{55}$ instead of absorption spectroscopy due to the lack of absorption in the region above $300 \mathrm{~nm}$. The st-PMMA/PLA complex films were obtained by evaporating the solvents in the condensed gels under reduced pressure at room 
temperature for $12 \mathrm{~h}$ followed by drying under vacuum at $130^{\circ} \mathrm{C}$ for $50 \mathrm{~h}$ and then used for the DSC and XRD measurements (Figures 3c,d and S8).

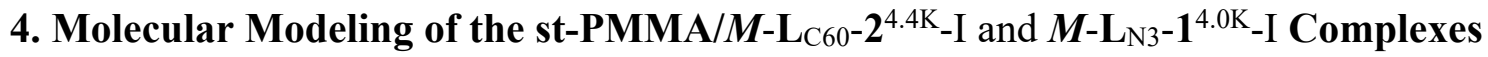

The molecular modeling was performed on a Windows 10 PC with the Materials Studio package (Version 8.0; Dassault Systèmes BIOVIA, San Diego, CA, USA). The initial structures of $\boldsymbol{M}$-L $\mathbf{L}_{\mathrm{C} 60^{-}}$

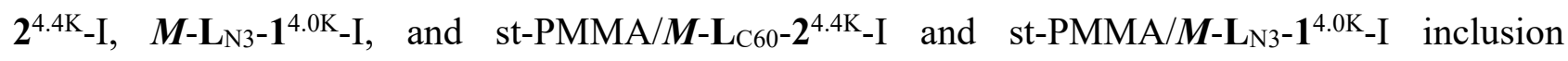
complexes were constructed according to the following procedures: first, the PLA segments of $\boldsymbol{M}$ -

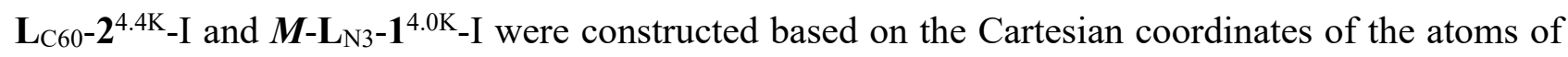
the lactide residue and the bond lengths, bond angles, and internal rotation angles reported for the $\alpha$ form poly(L-lactic acid) (PLLA) crystal. ${ }^{\mathrm{S} 6, \mathrm{~S} 7}$ The end groups of $\boldsymbol{M}-\mathbf{L}_{\mathrm{C} 60}-\mathbf{2}^{4.4 \mathrm{~K}_{-} \mathrm{I}}$ and $\boldsymbol{M}$ - $\mathbf{L}_{\mathrm{N} 3}-\mathbf{1}^{4.0 \mathrm{~K}_{-} \mathrm{I}}$ were then added to the PLA chains and selectively optimized by the molecular mechanics (MM) calculations (Compass II force field ${ }^{\mathrm{S} 8}$ as implemented in the Materials Studio package). An initial left-handed 18, $\boldsymbol{M}$-st-PMMA (576mer) helical model (18 monomer units/turn) was built according to the reported method. ${ }^{\mathrm{S} 9}$ The energy-minimized $\boldsymbol{M}-\mathbf{L}_{\mathrm{C} 60}-\mathbf{2}^{4.4 \mathrm{~K}_{-} \mathrm{I}}$ and $\boldsymbol{M}-\mathbf{L}_{\mathrm{N} 3}-\mathbf{1}^{4.0 \mathrm{~K}_{-} \mathrm{I}}$ were then manually inserted into the helical cavity of the $\boldsymbol{M}$-st-PMMA helical model to generate initial $\boldsymbol{M}$-st-PMMA/M-

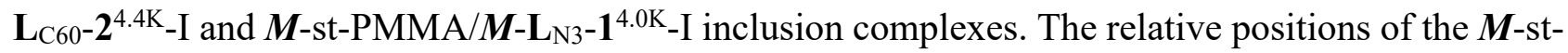
PMMA and $\boldsymbol{M}$ - $\mathbf{L}_{\mathrm{C} 60}-\mathbf{2}^{4.4 \mathrm{~K}_{-} \mathrm{I}}$ or $\boldsymbol{M}$ - $\mathbf{L}_{\mathrm{N} 3}-\mathbf{1}^{4.0 \mathrm{~K}_{-} \mathrm{I}}$ helices were determined in order to avoid any

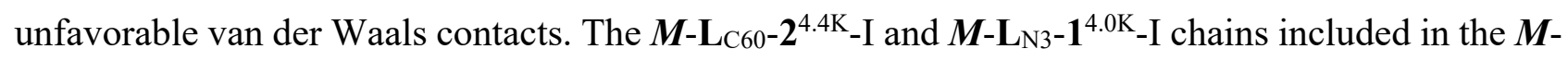
st-PMMA helix except for the PLA backbones were then selectively geometry-optimized by the MM calculations (Compass II force field) (Figure S10).

\section{General Procedures for VCD Measurements of st-PMMA Gels Complexed with the $\mathrm{C}_{60}$-Free and $\mathrm{C}_{60}$-Bound $M$ - and $P$-PLAs}

A typical experimental procedure is described below. st-PMMA $(8.0 \mathrm{mg})$ was dissolved in a

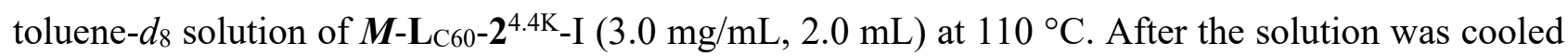
to room temperature, the solution gelled within $10 \mathrm{~min}$. The resulting soft gel was centrifuged at 1700 $g$ for 10 min and the supernatant containing the unencapsulated $\boldsymbol{M}-\mathbf{L}_{\mathrm{C} 60}-\mathbf{2}^{4.4 \mathrm{~K}}-\mathrm{I}$ was removed from the gel by decantation. The condensed gel was then washed with toluene- $d_{8}$ and the solvent was removed by decantation after centrifugation. This procedure was repeated two times $(0.2 \mathrm{~mL} \times 2)$. The obtained

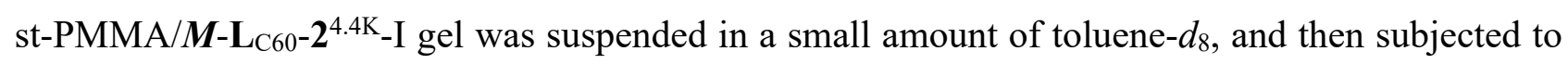


the IR and VCD measurements. In the same way, st-PMMA/P-D $\mathbf{C}_{60}-\mathbf{2}^{4.4 \mathrm{~K}}-\mathrm{F}$ and st-PMMA/rac-C $60^{-}$

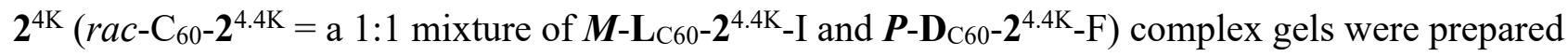
and their IR and VCD spectra were measured. The VCD spectrum of the st-PMMA/rac- $\mathrm{C}_{60}-\mathbf{2}^{4 \mathrm{~K}}$ complex gel was subtracted from the VCD spectra of the st-PMMA/M-L $\mathbf{L}_{\mathrm{C} 60}-\mathbf{2}^{4.4 \mathrm{~K}_{-}} \mathrm{I}$ and st-PMMA/P-

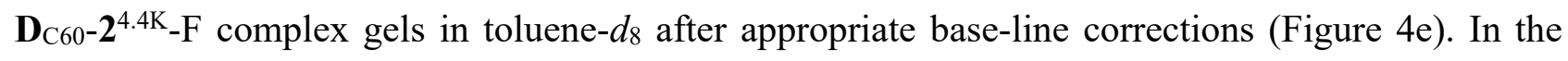

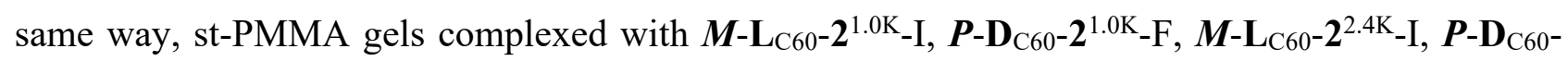

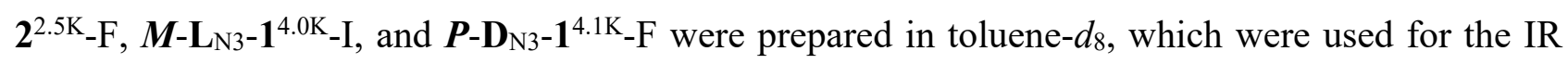
and VCD measurements (Figures $4 \mathrm{f}$ and S9h,i).

\section{Preparation of st-PMMA/M- and P-PLA Complex Gels in Toluene and Replacement with $\mathrm{C}_{60}$}

A typical experimental procedure is described below. st-PMMA $(8.0 \mathrm{mg})$ was dissolved in a toluene solution of $\boldsymbol{M}-\mathbf{L}_{\mathrm{C} 60}-\mathbf{2}^{4.4 \mathrm{~K}_{-} \mathrm{I}}(3.0 \mathrm{mg} / \mathrm{mL}, 2.0 \mathrm{~mL})$ at $110{ }^{\circ} \mathrm{C}$. After the solution was cooled to room temperature, the solution gelled within $10 \mathrm{~min}$. The obtained soft gel was collected by centrifugation at $1700 \mathrm{~g}$ for $10 \mathrm{~min}$ and the supernatant was removed from the gel by decantation. The condensed gel was then washed with toluene to remove the unencapsulated $\boldsymbol{M}$ - $\mathbf{L}_{\mathrm{C} 60}-\mathbf{2}^{4.4 \mathrm{~K}_{-} \mathrm{I}}$ by centrifugation followed by decantation. This procedure was repeated 2 times $(0.2 \mathrm{~mL} \times 2)$. The

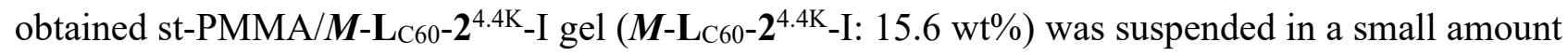
of toluene, and then subjected to the absorption and ECD measurements.

To replace the encapsulated $\boldsymbol{M}-\mathbf{L}_{\mathrm{C} 60}-\mathbf{2}^{4.4 \mathrm{~K}_{-}} \mathrm{I}(15.6 \mathrm{wt} \%)$ with the achiral $\mathrm{C}_{60}$, the st-PMMA/M-

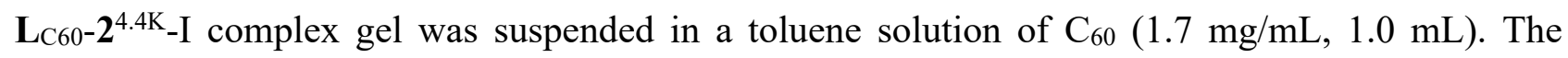
mixture was vigorously stirred at room temperature by a magnetic stirrer. After $30 \mathrm{~min}$, the obtained gel particles were recovered by centrifugation at $1700 \mathrm{~g}$ for $10 \mathrm{~min}$ and the supernatant containing

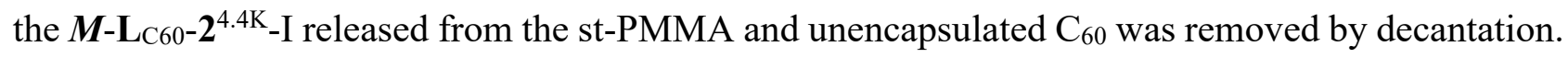
This procedure was repeated 10 times $(1.0 \mathrm{~mL} \times 10)$. The condensed gel was then washed with toluene and the solvent was removed by decantation after centrifugation. This procedure was repeated

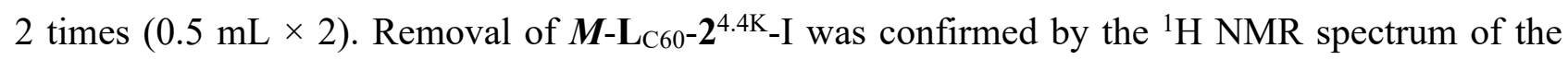
isolated st-PMMA/ $\mathrm{C}_{60}$ complex (Figure S12). The obtained gel was suspended in a small amount of toluene, and then subjected to the absorption and ECD measurements. 


\section{Helix-Sense-Selective Encapsulation of the Racemic $\mathrm{C}_{60}$-Bound and $\mathrm{C}_{60}$-Free $M$ - and $P$-PLAs with $M$ - and $P$-st-PMMA with a Helicity Memory}

An optically-active $M$ - or $P$-st-PMMA gel was prepared according to a previously reported method. ${ }^{\mathrm{S} 10, \mathrm{~S} 11}$ A typical experimental procedure is described below. st-PMMA (3.0 mg) was dissolved in $(R)$-1-phenylethylamine $((R)-3)(75 \mu \mathrm{L})$ at $110^{\circ} \mathrm{C}$. After the solution was cooled to $3{ }^{\circ} \mathrm{C}$, the solution gelled within $3 \mathrm{~h}$. The obtained gel was washed with toluene to remove $(R)-3$ completely by centrifugation followed by decantation. This procedure was repeated five times $(0.15 \mathrm{~mL} \times 7)$, affording an optically-active $M$-st-PMMA with helicity memory. The gel was then suspended in a

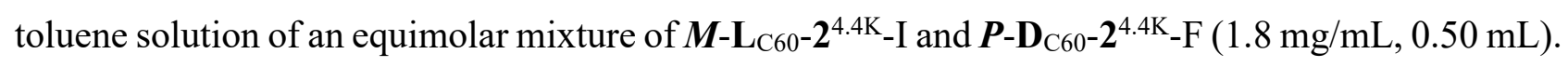
The mixture was vigorously stirred at room temperature for $3 \mathrm{~h}$ by a magnetic stirrer, thus producing soft gel particles. The obtained $M$-st-PMMA/nonracemic $\mathrm{C}_{60}-\mathbf{2}^{4.4 \mathrm{~K}}$ complex gel was collected by centrifugation at $1700 \mathrm{~g}$ for $10 \mathrm{~min}$ and the supernatant containing the unencapsulated $\mathrm{C}_{60}-\mathbf{2}^{4.4 \mathrm{~K}}$ was recovered by decantation. The encapsulated $\mathrm{C}_{60}-\mathbf{2}^{4.4 \mathrm{~K}}$ content was estimated from the integral ratio of the methoxy and methine protons of the $M$-st-PMMA and $\mathrm{C}_{60}-\mathbf{2}^{4.4 \mathrm{~K}}$ in the ${ }^{1} \mathrm{H}$ NMR spectrum, respectively, and also determined from absorption spectroscopy as described in Section 3. After evaporation of the solvents in the $M$-st-PMMA/ $\mathrm{C}_{60}-2^{4.4 \mathrm{~K}}$ complex gel and of its supernatant, the residues were dissolved in $\mathrm{CDCl}_{3}$, and the ee values of the encapsulated and unencapsulated $\mathrm{C}_{60}-2^{4.4 \mathrm{~K}}$ were determined by ${ }^{1} \mathrm{H}$ NMR (Figure 6a(ii,iii)). In the same way, the helix-sense-selective encapsulations of other $\mathrm{C}_{60}$-bound $r a c-2$ and $\mathrm{C}_{60}$-free $r a c-1$ with different molar masses were conducted using the $M$ - and $P$-helical st-PMMA gels with helicity memory prepared by $(R)$ - and $(S)$ $\mathbf{3}$, respectively, and the results are summarized in Table 3. 


\section{PLA Chain Exchange Reactions Between the $\mathrm{C}_{60}$-Bound Helical $M$ - or $P$-PLAs Encapsulated in the $M$-st-PMMA Helical Cavity and Free $\mathrm{C}_{60}$-Bound Helical $M$ - or $P$-PLAs}

An optically-active $M$-st-PMMA gel with helicity memory was prepared in $(R)$-3 as described in Section 7. The gel consisting of $M$-st-PMMA $(6.0 \mathrm{mg})$ was then suspended in a toluene solution of $\boldsymbol{M}$ - $\mathbf{L}_{\mathrm{C} 60}-\mathbf{2}^{4.4 \mathrm{~K}_{-} \mathrm{I}}(1.8 \mathrm{mg} / \mathrm{mL}, 0.50 \mathrm{~mL})$. The mixture was vigorously stirred at room temperature for 3 h by a magnetic stirrer, thus producing soft gel particles. The obtained $M$-st-PMMA/M-L $\mathbf{L}_{C 60}-\mathbf{2}^{4.4 \mathrm{~K}_{-} \mathrm{I}}$ complex gel was collected by centrifugation at $1700 \mathrm{~g}$ for $10 \mathrm{~min}$ and the supernatant containing the unencapsulated $\boldsymbol{M}$ - $\mathbf{L}_{\mathrm{C} 60}-\mathbf{2}^{4.4 \mathrm{~K}_{-} \mathrm{I}}$ was recovered by decantation. The encapsulated $\boldsymbol{M}$ - $\mathbf{L}_{\mathrm{C} 60}-\mathbf{2}^{4.4 \mathrm{~K}_{-} \mathrm{I}}$ content was determined from absorption spectroscopy as described in Section 3. The supernatant solution and the $M$-st-PMMA/M-L $\mathbf{L}_{\mathrm{C} 60}-\mathbf{2}^{4.4 \mathrm{~K}}-\mathrm{I}$ complex gel were then mixed again, followed by adding

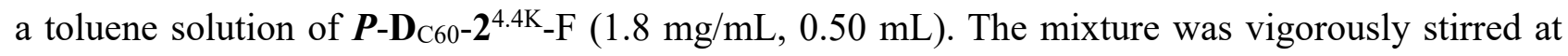
room temperature for $3 \mathrm{~h}$ by a magnetic stirrer. The obtained $M$-st-PMMA/nonracemic $\mathrm{C}_{60}-\mathbf{2}^{4.4 \mathrm{~K}}$ complex gel was collected by centrifugation at $1700 \mathrm{~g}$ for $10 \mathrm{~min}$ and the supernatant containing the unencapsulated $\mathrm{C}_{60}-2^{4.4 \mathrm{~K}}$ was recovered by decantation. The encapsulated $\mathrm{C}_{60}-\mathbf{2}^{4.4 \mathrm{~K}}$ content was estimated by ${ }^{1} \mathrm{H}$ NMR spectroscopy as described in Section 7. After evaporation of the solvents in the $M$-st-PMMA/ $\mathrm{C}_{60}-\mathbf{2}^{4.4 \mathrm{~K}}$ complex gel and of its supernatant, the residues were dissolved in $\mathrm{CDCl}_{3}$, and the ee values of the encapsulated and unencapsulated $\mathrm{C}_{60}-\mathbf{2}^{4.4 \mathrm{~K}}$ were determined by ${ }^{1} \mathrm{H} \mathrm{NMR}$. In the same way, the PLA chain exchange reaction between $\boldsymbol{P}-\mathbf{D}_{\mathrm{C} 60}-\mathbf{2}^{4.4 \mathrm{~K}}-\mathrm{F}$ encapsulated in the $\boldsymbol{M}$-stPMMA helical cavity and free $\boldsymbol{M}-\mathbf{L}_{\mathrm{C} 60}-\mathbf{2}^{4.4 \mathrm{~K}_{-} \mathrm{I}}$ were conducted, and the results are summarized in Figure S14a,b. 


\section{Helix-Sense-Selective Encapsulation of the Nonracemic $\mathrm{C}_{60}$-Bound PLA with $\boldsymbol{M}$ - and $\boldsymbol{P}$-st- PMMA with a Helicity Memory}

An optically-active $M$-st-PMMA gel with helicity memory was prepared in $(R)$-3 as described in Section 7. The gel consisting of $M$-st-PMMA $(15.0 \mathrm{mg})$ was then suspended in a toluene solution of

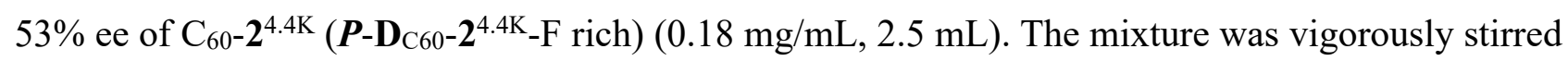
at room temperature for $3 \mathrm{~h}$ by a magnetic stirrer, thus producing soft gel particles. The obtained $M$ st-PMMA/nonracemic $\mathrm{C}_{60}-\mathbf{2}^{4.4 \mathrm{~K}}$ complex gel was collected by centrifugation at $1700 \mathrm{~g}$ for $10 \mathrm{~min}$ and the supernatant containing the unencapsulated $\mathrm{C}_{60}-\mathbf{2}^{4.4 \mathrm{~K}}$ was recovered by decantation. The encapsulated $\mathrm{C}_{60}-2^{4.4 \mathrm{~K}}$ content was estimated by ${ }^{1} \mathrm{H}$ NMR and absorption spectroscopies as described in Section 7. After evaporation of the solvents in the $M$-st-PMMA/ $\mathrm{C}_{60}-\mathbf{2}^{4.4 \mathrm{~K}}$ complex gel and of its supernatant, the residues were dissolved in $\mathrm{CDCl}_{3}$, and the ee values of the encapsulated and unencapsulated $\mathrm{C}_{60}-\mathbf{2}^{4.4 \mathrm{~K}}$ were determined by ${ }^{1} \mathrm{H}$ NMR (Figure $7 \mathrm{~b}$ (ii,iii)). 


\section{Supporting Data}

(a)

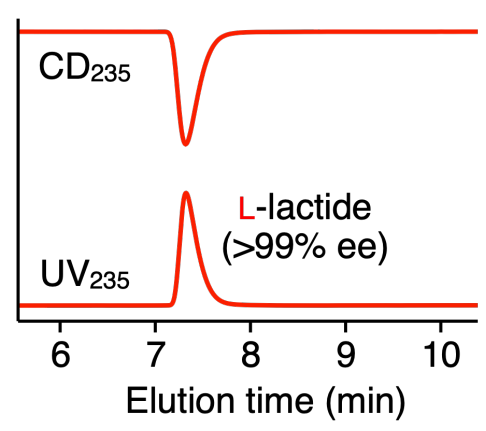

(b)

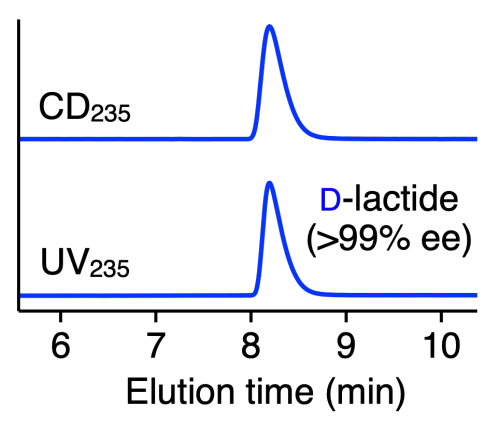

Figure S1. Verification of high optical purities ( $>99 \%$ ee) of L-lactide (a) and D-lactide (b) by chiral HPLC on a CHIRALPAK IA-3 column $(15 \mathrm{~cm} \times 0.46 \mathrm{~cm}$ (i.d.)). Chromatograms show UV (bottom) and CD (top) traces recorded at $235 \mathrm{~nm}$. Eluent, hexane/2-propanol (90/10, v/v); flow rate, 1.0 $\mathrm{mL} / \mathrm{min}$; temperature, ca. $20^{\circ} \mathrm{C}$. 


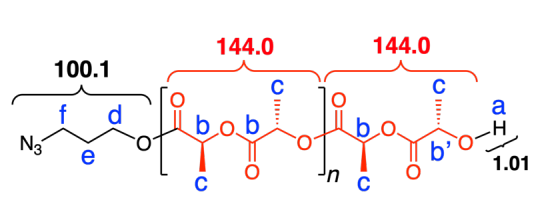

$M-\mathrm{N}_{3}-\mathrm{PLLA}{ }^{0.9 \mathrm{~K}}$

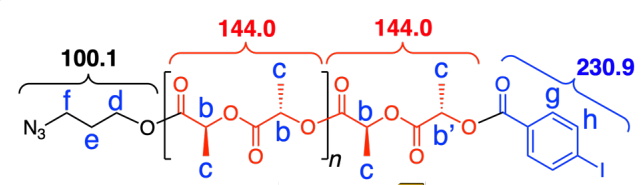

$M-L_{N 3}-11.0 K_{-1}$ N 10

(a) ${ }^{1} \mathrm{H}$ NMR
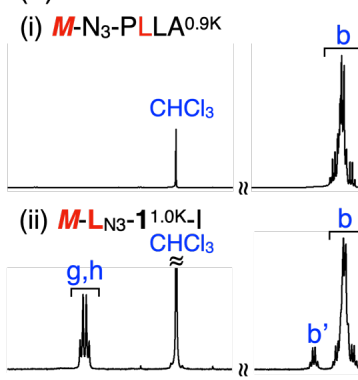

(iii) $M-L_{C 60}-2^{1 \cdot 0 K-I}$
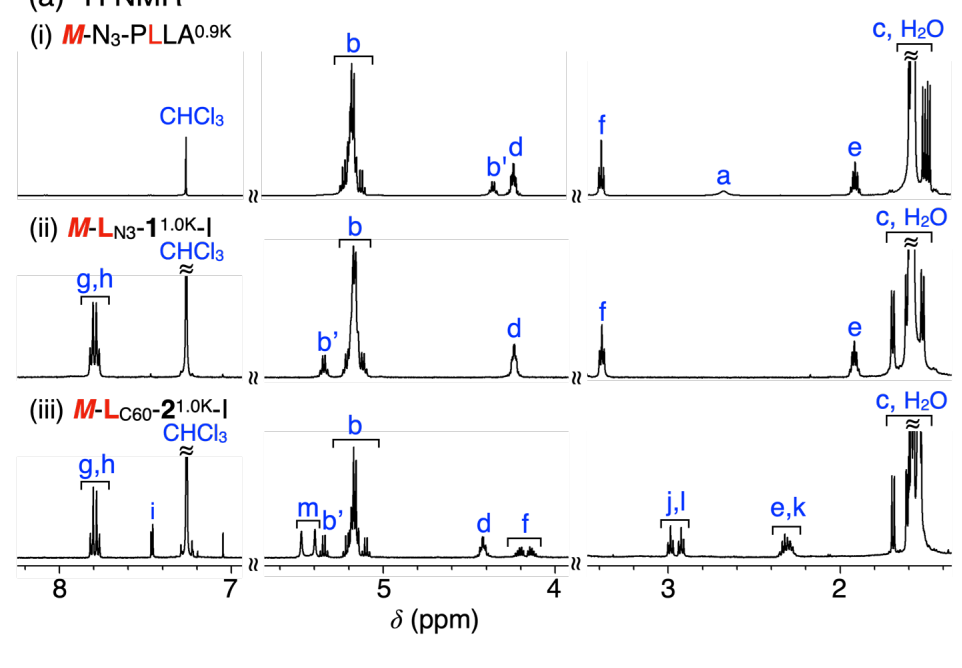

c, $\mathrm{H}_{2} \mathrm{O}$

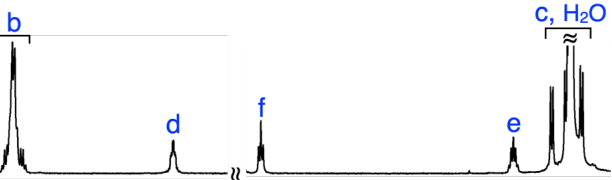

(b) MALDI-TOF-MS

(iv) $M-\mathrm{N}_{3}-\mathrm{PLLA}{ }^{0.9 \mathrm{~K}} \quad\left[\mathrm{M}-\mathrm{N}_{3}-\mathrm{PLLA}+\mathrm{Na}\right]^{+}$
M-LC60-2 ${ }^{1.0 \mathrm{~K}-\mathrm{I}}$

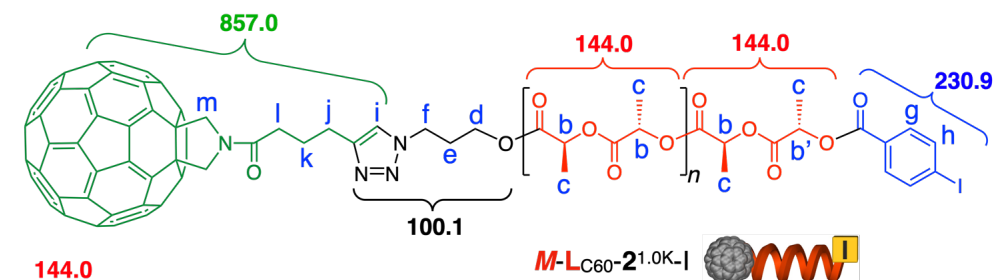

30.9

(v) $M-L_{N 3}-1^{1.0 K}-I$

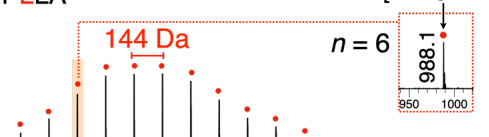

(vi) $M-L_{C 60}-2^{1.0 K-I}$

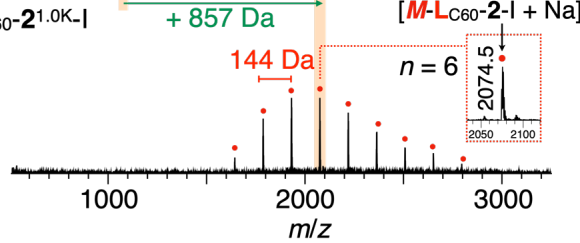

Figure S2. (a) ${ }^{1} \mathrm{H}$ NMR and (b) MALDI-TOF-MS spectra of $\boldsymbol{M}-\mathrm{N}_{3}-\mathrm{PLLA}^{0.9 \mathrm{~K}}, \boldsymbol{M}-\mathbf{L}_{\mathrm{N} 3}-\mathbf{1}^{1.0 \mathrm{~K}}-\mathrm{I}$, and $\boldsymbol{M}$ -

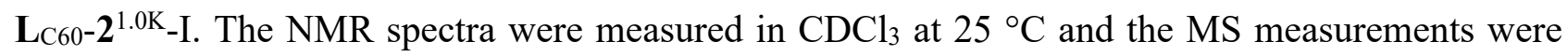
performed using dithranol as a matrix and sodium iodide as a cationizing agent. 


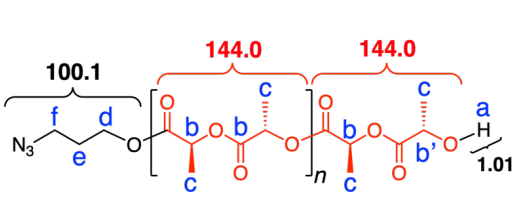

$M-\mathrm{N}_{3}-\mathrm{PLLA} 2.1 \mathrm{~K}$

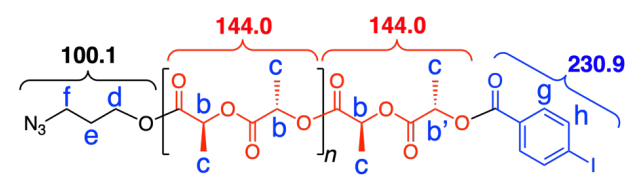

$\left.M-L_{N 3}-12.3 K_{-1} N_{3}\right) N$

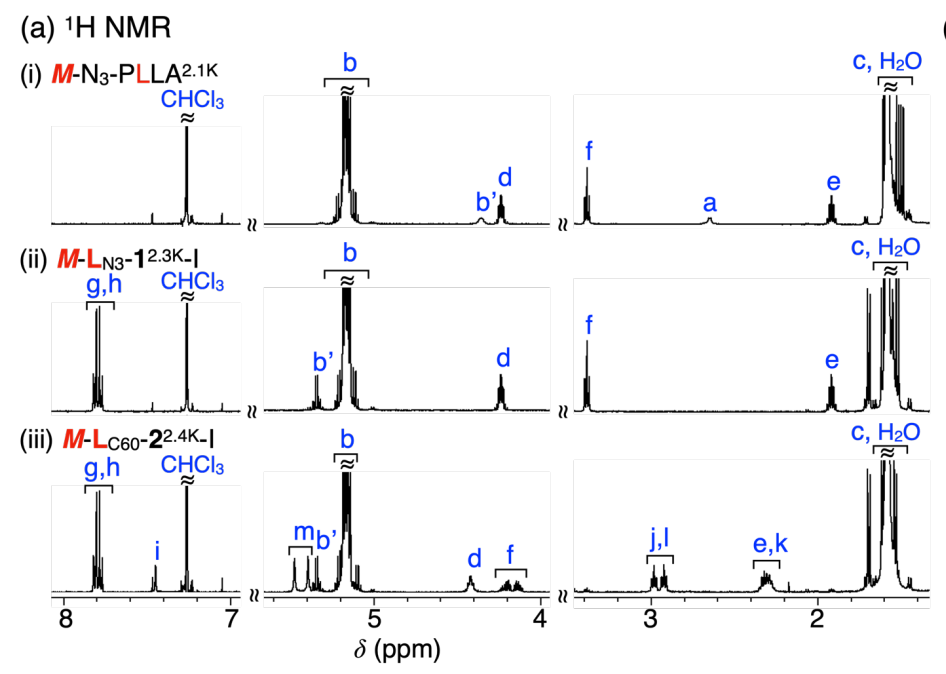

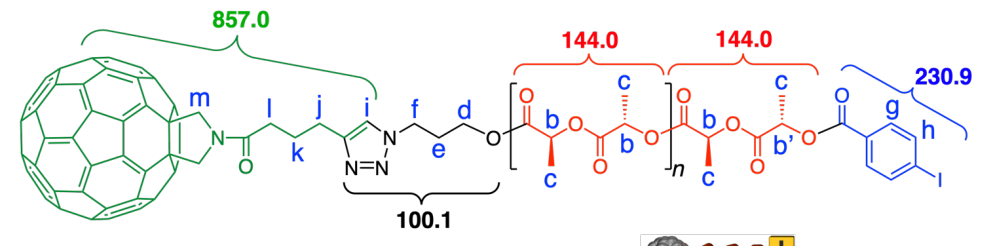

$M-L_{C 60}-2^{2.4 K-I}$

arm

(b) MALDI-TOF-MS

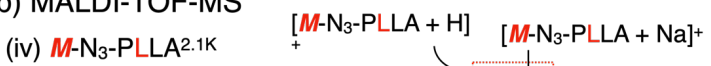

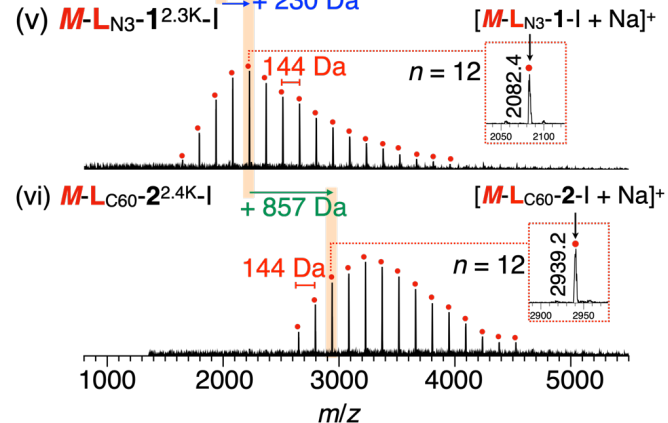

Figure S3. (a) ${ }^{1} \mathrm{H}$ NMR and (b) MALDI-TOF-MS spectra of $\boldsymbol{M}$-N $3-\mathrm{PLLA}^{2.1 \mathrm{~K}}, \boldsymbol{M}-\mathbf{L}_{\mathrm{N} 3}-\mathbf{1}^{2.3 \mathrm{~K}}$-I, and $\boldsymbol{M}$ -

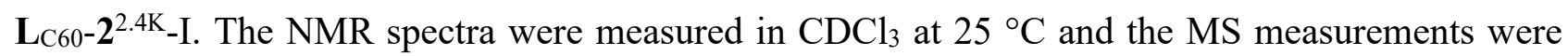
performed using dithranol as a matrix and sodium iodide as a cationizing agent. 


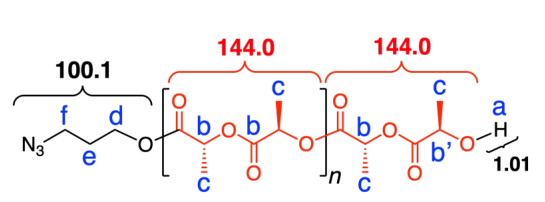

$\boldsymbol{P}^{-N_{3}}$-PDLA ${ }^{0.9 K}$

(a) ${ }^{1} \mathrm{H}$ NMR

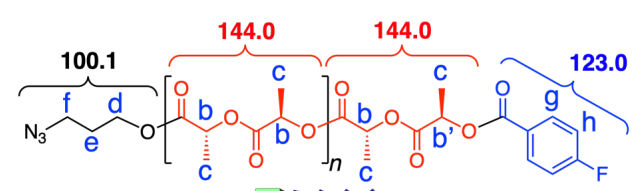

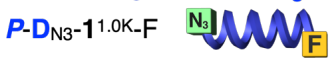

(i) $P-\mathrm{N}_{3}-\mathrm{PDLA} \mathrm{A}^{0.9 \mathrm{~K}}$

(ii) $P$-D $\mathrm{N}-1$ 1.0K-

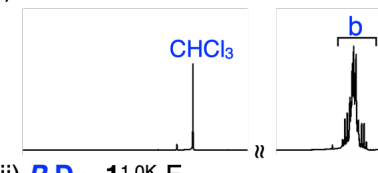

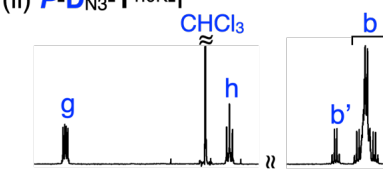

(iii) $\boldsymbol{P}$-DC60-2 ${ }^{1.0 K_{-}} \mathrm{F}$

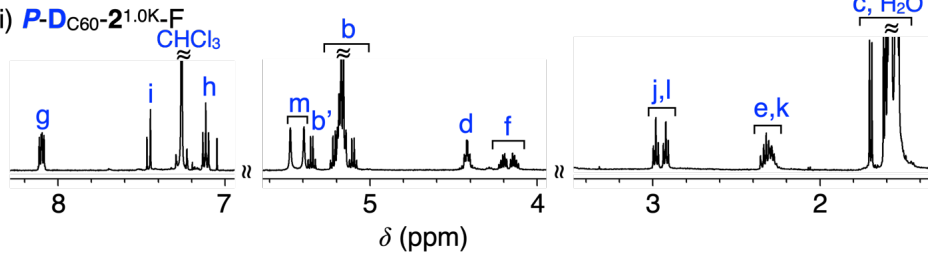

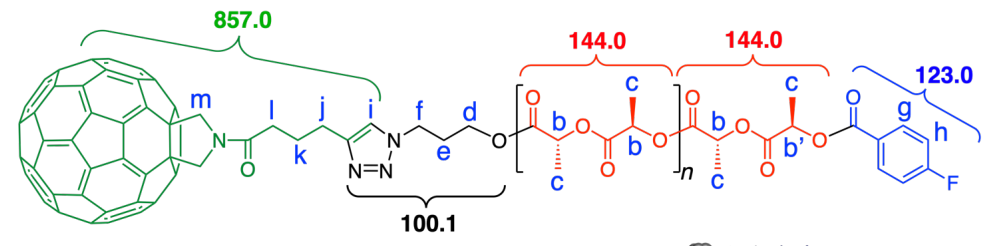

$P-D_{\mathrm{C} 60-2}{ }^{1.0 \mathrm{~K}}-\mathrm{F}$

(b) MALDI-TOF-MS

(iv) $P$ - $\mathrm{N}_{3}-\mathrm{PDLA} \mathrm{A}^{0.9 \mathrm{~K}}$

$\left[P-\mathrm{N}_{3}-\mathrm{PDLA}+\mathrm{Na}\right]^{+}$

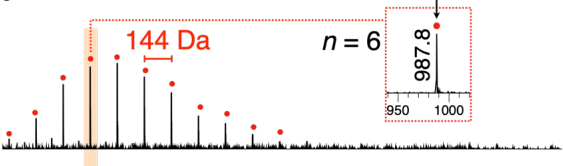

(v) $P$ - $D_{\mathrm{N} 3}-1^{1.0 K}-\mathrm{F}$

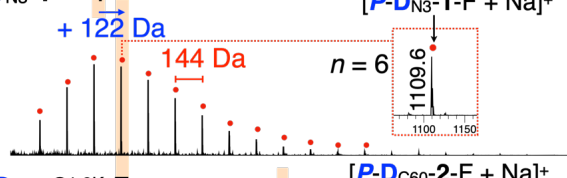

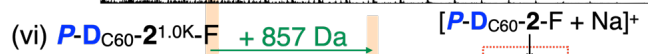

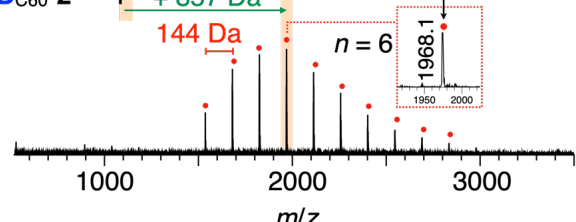

Figure S4. (a) ${ }^{1} \mathrm{H}$ NMR and (b) MALDI-TOF-MS spectra of $\boldsymbol{P}-\mathrm{N}_{3}-\mathrm{PDLA}^{0.9 \mathrm{~K}}, \boldsymbol{P}-\mathbf{D}_{\mathrm{N} 3}-\mathbf{1}^{1.0 \mathrm{~K}}-\mathrm{F}$, and $\boldsymbol{P}$ $\mathbf{D}_{\mathrm{C} 60-2} 2^{1.0 \mathrm{~K}}-\mathrm{F}$. The NMR spectra were measured in $\mathrm{CDCl}_{3}$ at $25{ }^{\circ} \mathrm{C}$ and the MS measurements were performed using dithranol as a matrix and sodium iodide as a cationizing agent. 


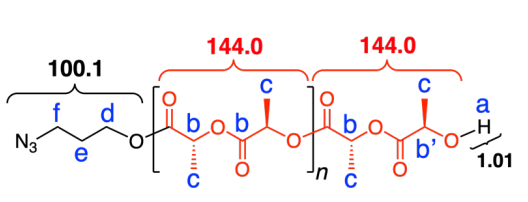

P-N $-\mathrm{NDLA}^{2.3 \mathrm{~K}}$

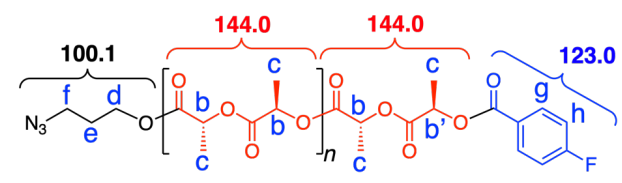

$P-D_{\mathrm{N} 3}-12.4 \mathrm{~K}-\mathrm{F} \quad \sqrt[\mathrm{N}_{3}]{1}$

(a) ${ }^{1} \mathrm{H}$ NMR

(i) $P$ - $\mathrm{N}_{3}-\mathrm{PDLA}$

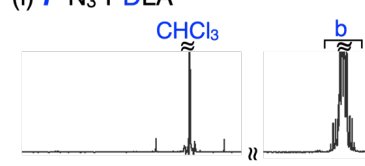

(ii) $P$ - $\mathrm{DN}_{\mathrm{N} 3}-12.4 \mathrm{~K}-\mathrm{F}$

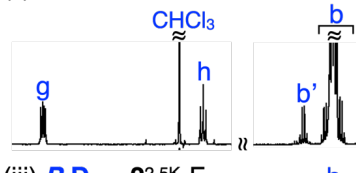

(iii) $P$-D $60-2$ 2.5K-F
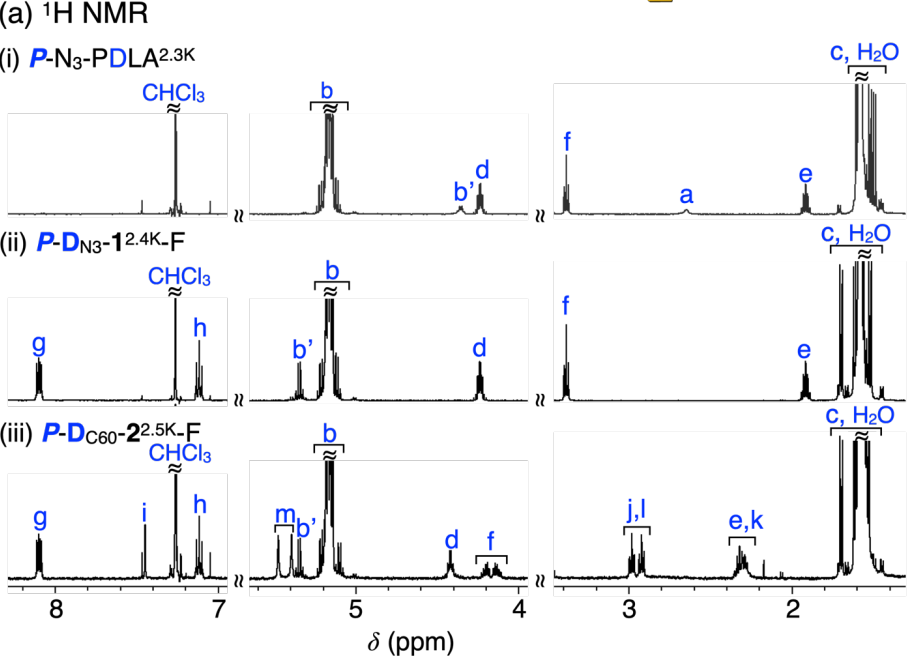

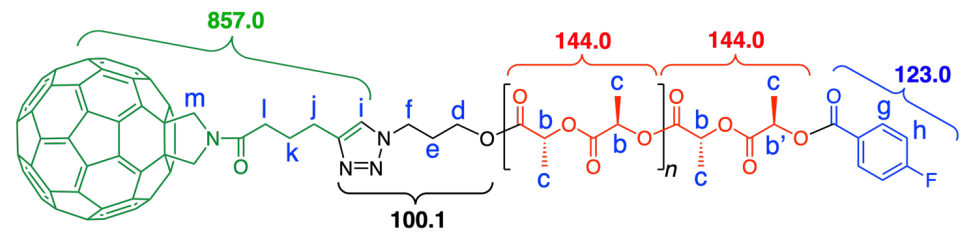

$P$-DC60-22.5K-F

a $\mathrm{un}_{\mathrm{F}}$

(b) MALDI-TOF-MS

$\left[P-\mathrm{N}_{3}-\mathrm{PDLA}+\mathrm{Na}\right]^{+}$

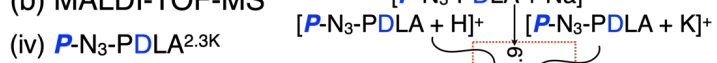

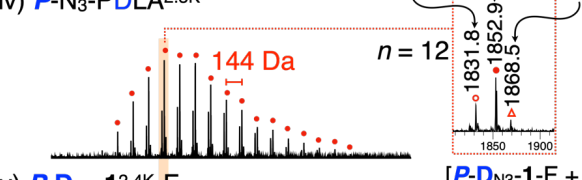

.
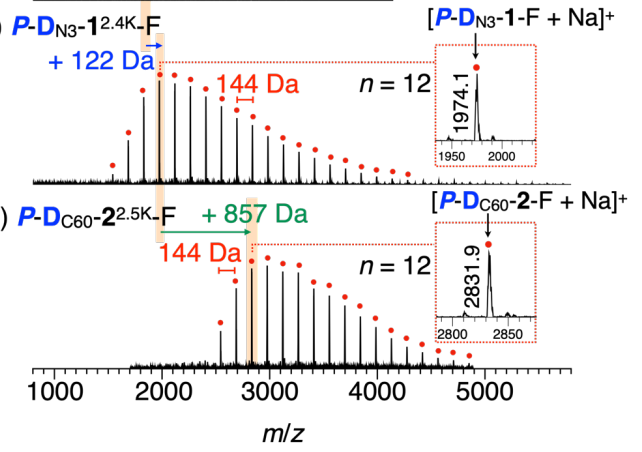

Figure S5. (a) ${ }^{1} \mathrm{H}$ NMR and (b) MALDI-TOF-MS spectra of $\boldsymbol{P}-\mathrm{N}_{3}-\mathrm{PDLA}^{2.3 \mathrm{~K}}, \boldsymbol{P}-\mathbf{D}_{\mathrm{N} 3}-\mathbf{1}^{2.4 \mathrm{~K}}-\mathrm{F}$, and $\boldsymbol{P}$ $\mathbf{D}_{\mathrm{C} 60-2^{2.5}}-\mathrm{F}$. The NMR spectra were measured in $\mathrm{CDCl}_{3}$ at $25{ }^{\circ} \mathrm{C}$ and the $\mathrm{MS}$ measurements were performed using dithranol as a matrix and sodium iodide as a cationizing agent. 


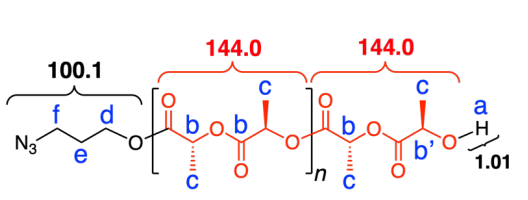

P-N $-\mathrm{NDLA}^{4.0 \mathrm{~K}}$

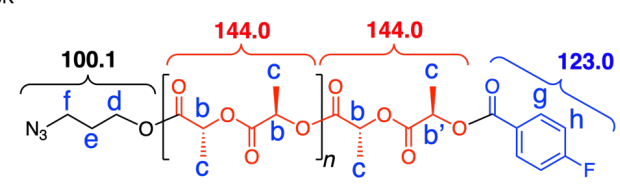

$P$-D $\left.{ }_{\mathrm{N} 3}-14.1 \mathrm{~K}-\mathrm{F} \mathrm{N}_{3}\right]$

(a) ${ }^{1} \mathrm{H}$ NMR

(i) $\boldsymbol{P}-\mathrm{N}_{3}-\mathrm{PDLA} 4.0 \mathrm{~K}$

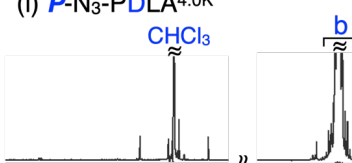

(ii) $P$ - $\mathrm{D}_{\mathrm{N} 3}-1^{4.1 \mathrm{~K}}-\mathrm{F}$

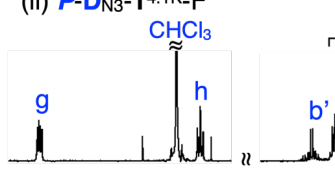

(iii) $P$-D $\mathrm{C} 60-2$-4K-F
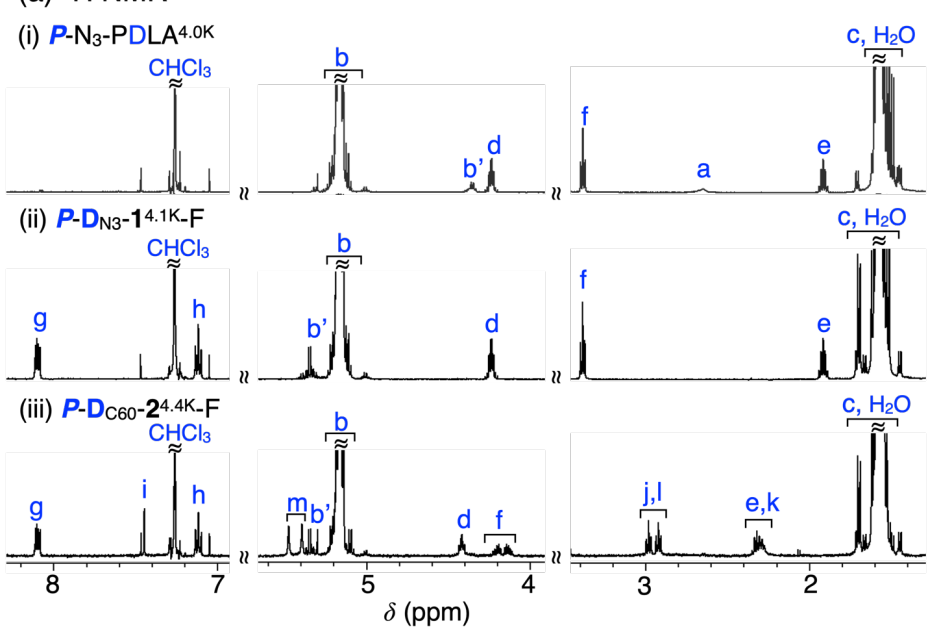

C, $\mathrm{H}_{2} \mathrm{O}$

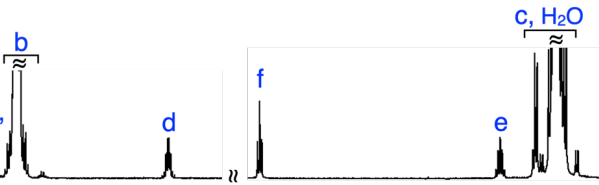

(v)

(b) MALDI-TOF-MS

$\left[P-\mathrm{N}_{3}-\mathrm{PDLA}+\mathrm{Na}\right]^{+}$

(iv) $P-\mathrm{N}_{3}-\mathrm{PDLA}{ }^{4.0 \mathrm{~K}}$

P-D 60 -2 4 .4K-F

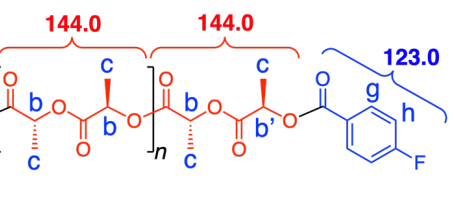

Qun

P-DN3-14.1K-F

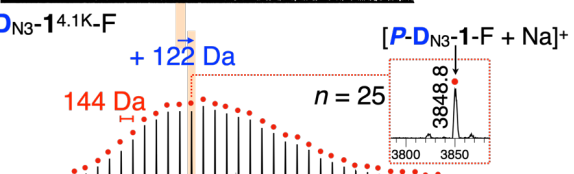

(vi) $P$-D $600-2^{4.4 K-F}$

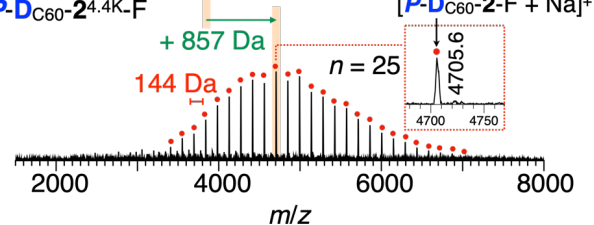

Figure S6. (a) ${ }^{1} \mathrm{H}$ NMR and (b) MALDI-TOF-MS spectra of $\boldsymbol{P}$-N 3 -PDLA ${ }^{4.0 \mathrm{~K}}, \boldsymbol{P}$ - $\mathbf{D}_{\mathrm{N} 3}-\mathbf{1}^{4} .1 \mathrm{~K}-\mathrm{F}$, and $\boldsymbol{P}$ -

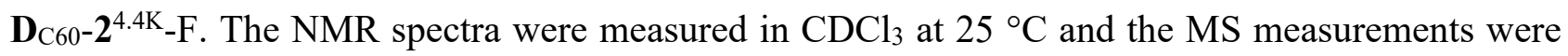
performed using dithranol as a matrix and sodium iodide as a cationizing agent.

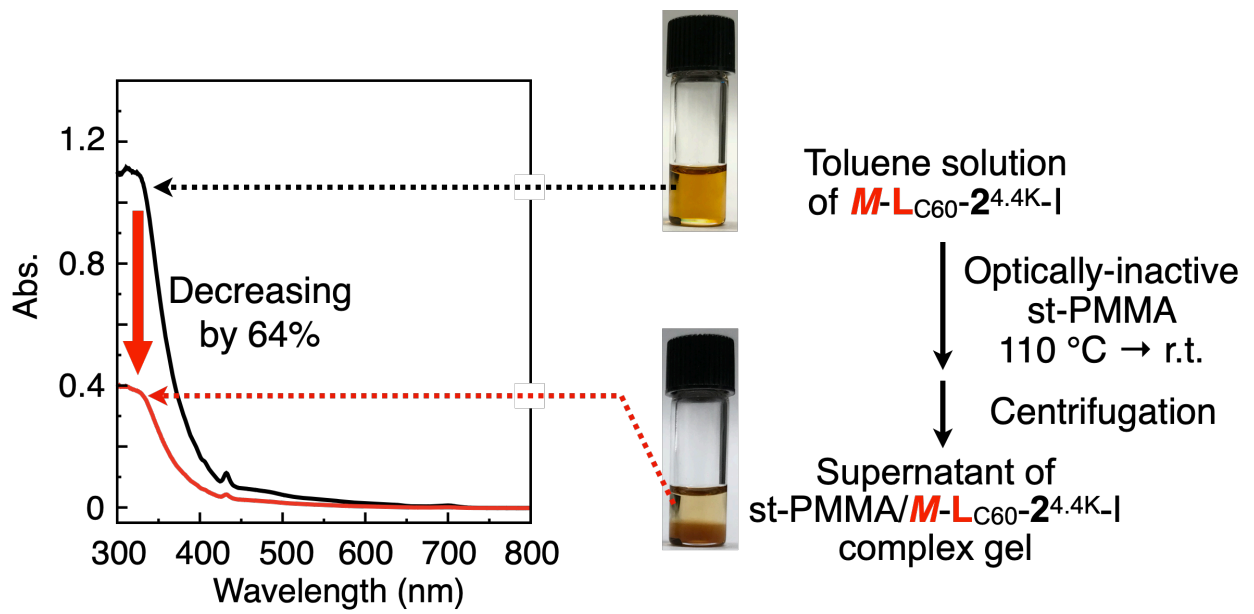

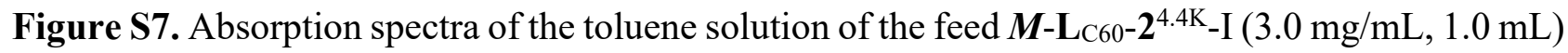

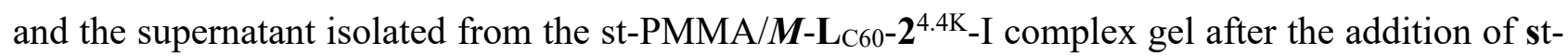
PMMA (10 mg) with subsequent heating at $110{ }^{\circ} \mathrm{C}$ followed by cooling to r.t. and then centrifuged at $1700 \mathrm{~g}$ for $10 \mathrm{~min}$. 


\section{(a) DSC}

$$
\text { st-PMMA/M-LC60-24.4K-I film }
$$

(i) 1st heating (M-LC60-24.4K-I: $23.5 \mathrm{wt} \%)$

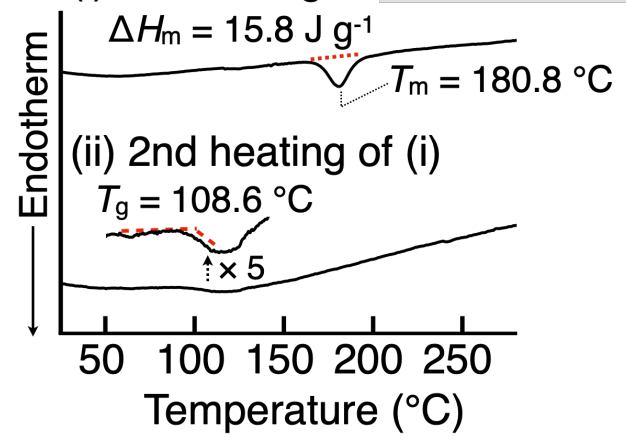

(b) XRD

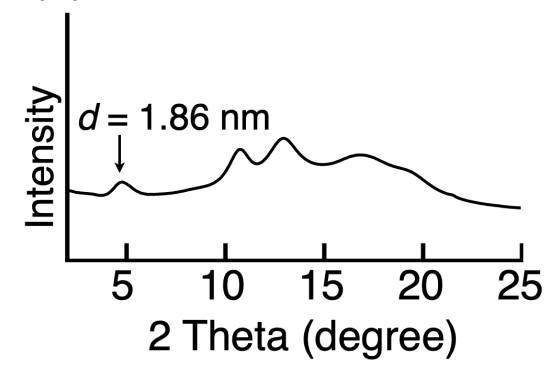

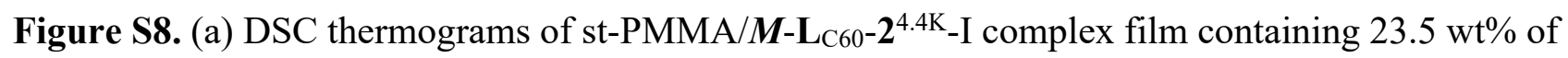
$\boldsymbol{M}$ - $\mathrm{L}_{\mathrm{C} 60-2^{4}}{ }^{4 \mathrm{~K}}-\mathrm{I}$ (i). The film was prepared by evaporating the solvent from the st-PMMA $/ \boldsymbol{M}-\mathbf{L}_{\mathrm{C} 60^{-}}$ $2^{4.4 \mathrm{~K}}$-I complex gel in toluene. The DSC measurement was conducted after cooling the sample at $20^{\circ} \mathrm{C}$, followed by heating to $280^{\circ} \mathrm{C}\left(10^{\circ} \mathrm{C} / \mathrm{min}\right)$ under nitrogen. The sample (i) was then cooled to $-20^{\circ} \mathrm{C}\left(40{ }^{\circ} \mathrm{C} / \mathrm{min}\right)$, and then heated again ((ii); $\left.10^{\circ} \mathrm{C} / \mathrm{min}\right)$. The arrow to the left of the DSC data

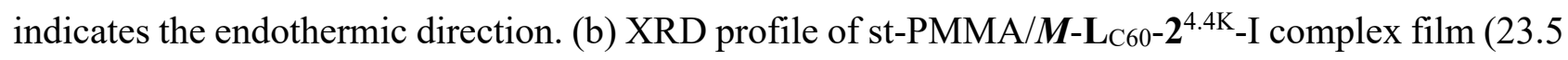
wt $\%)$. 
(a) Left-handed helix rich

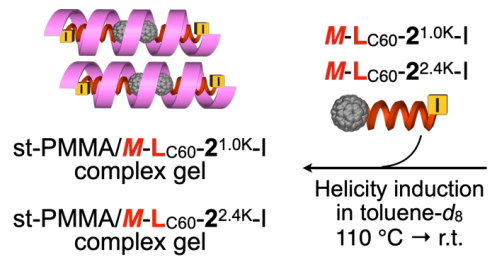

Right-handed helix rich

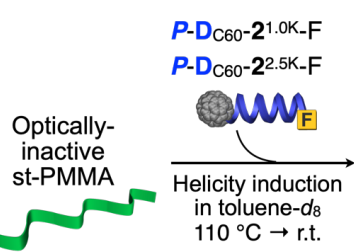

(d)
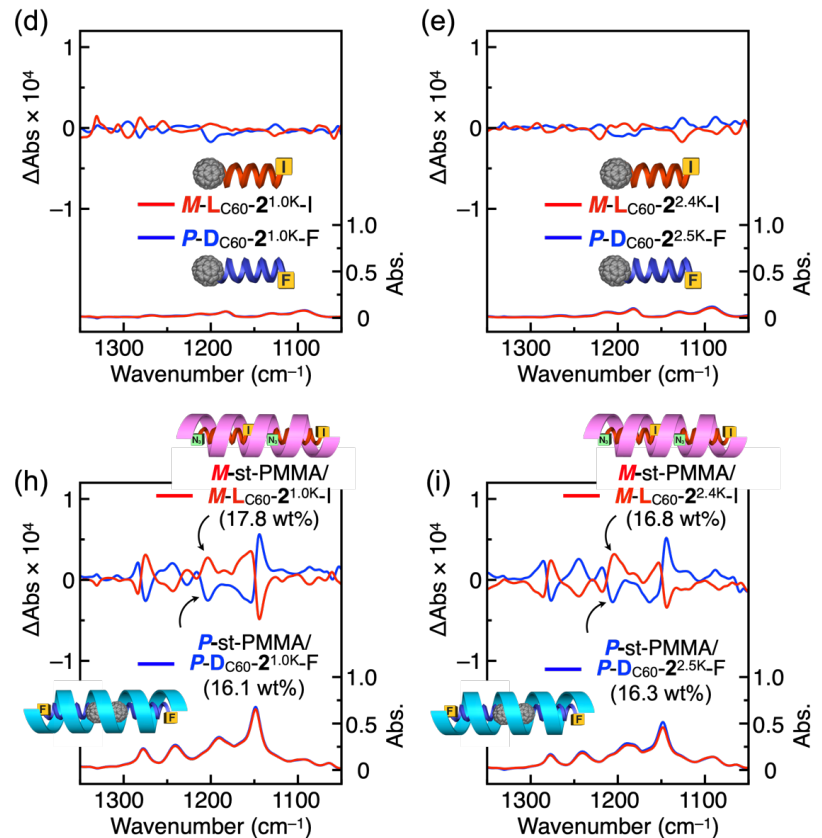

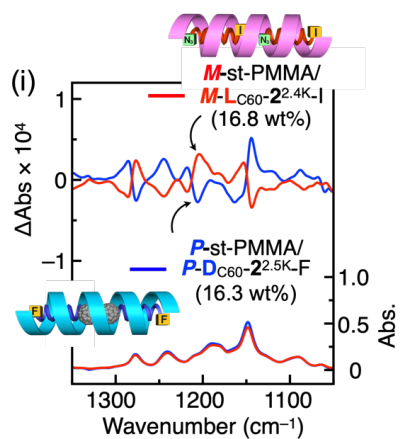

Figure S9. (a) Schematic illustration of $M$ - (left) and $P$ - (right) helicity induction in st-PMMA upon encapsulation of $\boldsymbol{M}-\mathbf{L}_{\mathrm{C} 60-2}-\mathrm{I}$ and $\boldsymbol{P}-\mathbf{D}_{\mathrm{C} 60-2-F}$, respectively. (b,f) ECD (top) and absorption (bottom)

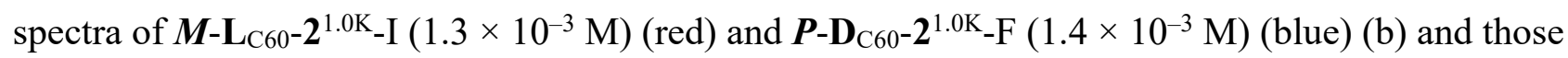

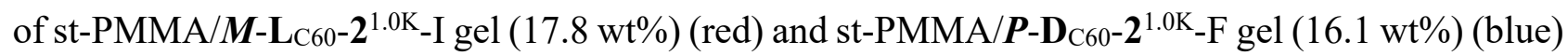

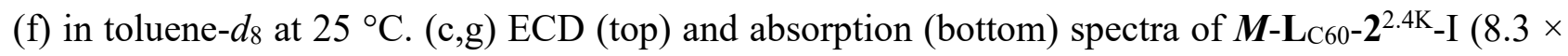

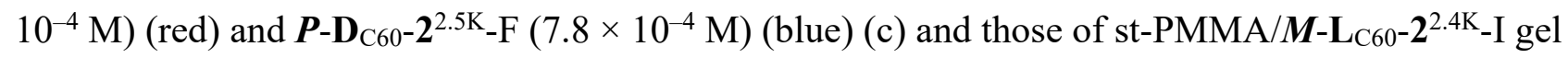
$(16.8 \mathrm{wt} \%)$ (red) and st-PMMA/P-D $\mathbf{C}_{60}-2^{2.5 \mathrm{~K}}-\mathrm{F}$ gel (16.3 wt\%) (blue) (g) in toluene- $d_{8}$ at $25^{\circ} \mathrm{C}$. The ECD and absorption spectra in (b,c,f,g) were normalized based on the corresponding absorption spectra at $25^{\circ} \mathrm{C}$. The contribution of the linear dichroism caused by the macroscopic anisotropy was

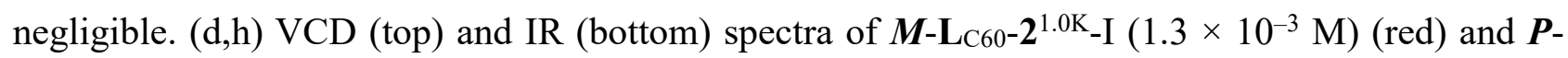

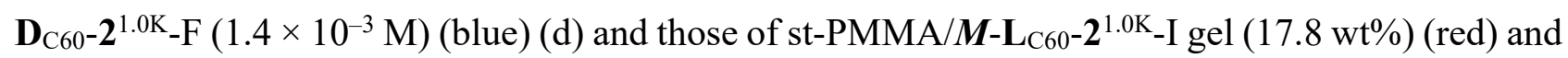
st-PMMA/P-D ${ }_{\mathrm{C} 60-2^{1.0 K}}-\mathrm{F}$ gel (16.1 wt\%) (blue) complex gels (h) in toluene- $d_{8}$ at room temperature.

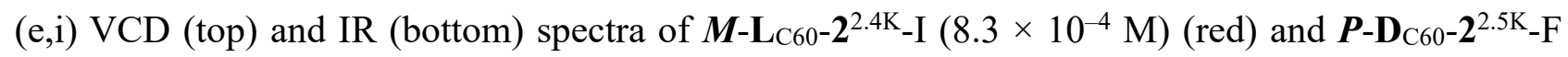

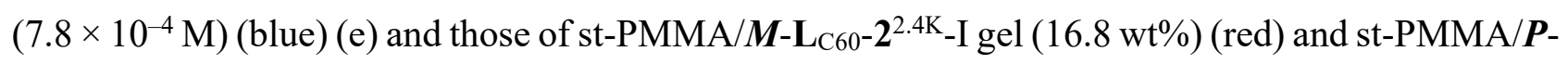

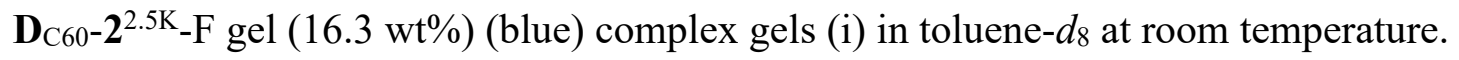




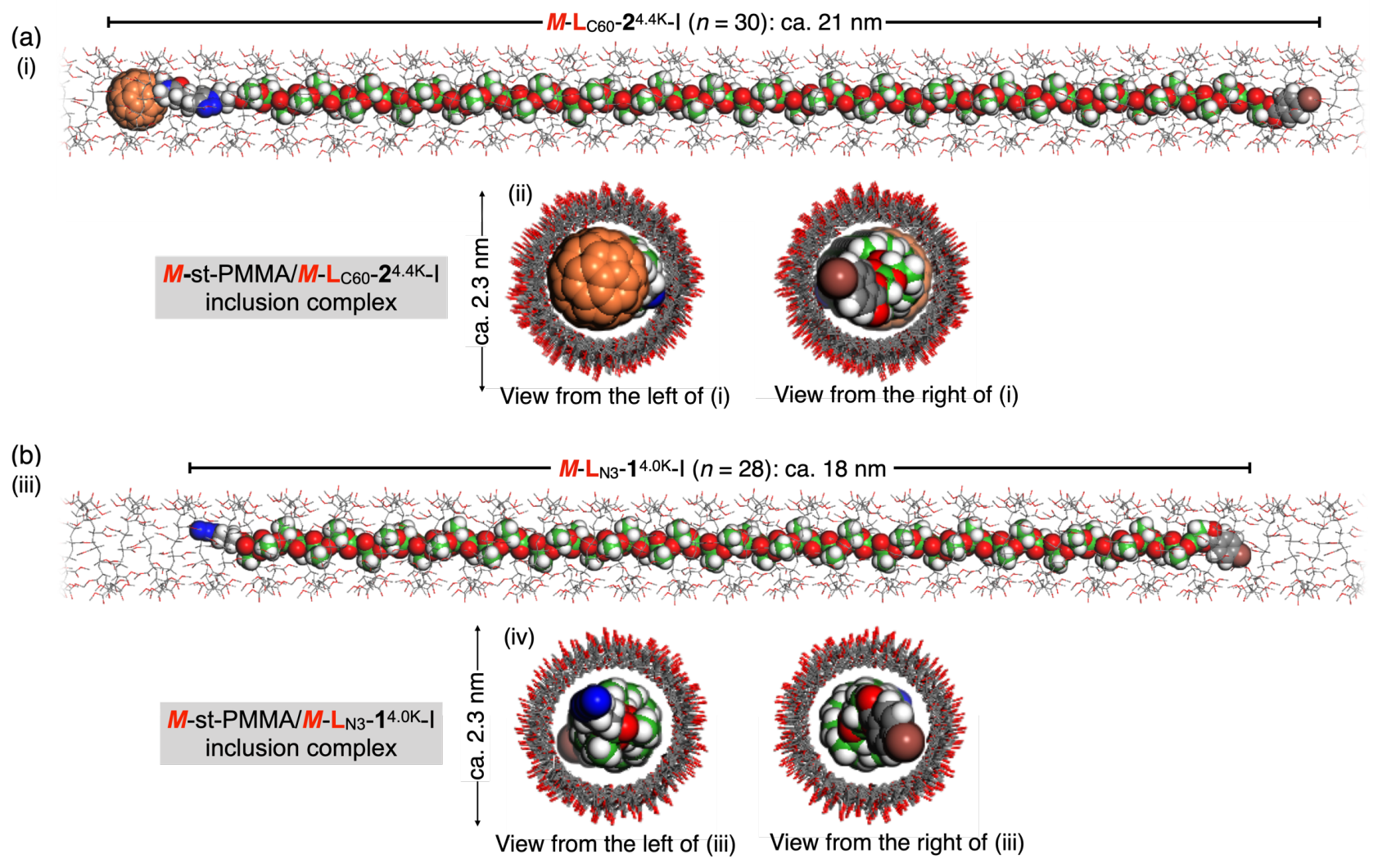

Figure S10. Side (i and iii) and top (ii and iv) views of the geometry-optimized structures of the inclusion complexes between the left-handed $18_{1}$ helical $\boldsymbol{M}$-st-PMMA and the left-handed $10_{3}$ helical $\boldsymbol{M}$ - $\mathbf{L}_{\mathrm{C} 60}-\mathbf{2}^{4.4 \mathrm{~K}_{-} \mathrm{I}}$ (a) and the left-handed $10_{3}$ helical $\boldsymbol{M}-\mathbf{L}_{\mathrm{N} 3}-\mathbf{1}^{4.0 \mathrm{~K}_{-} \mathrm{I}}$ (b) obtained by the molecular mechanics (MM) calculations (Compass II force field). Hydrogen atoms of st-PMMA are omitted for clarity. The carbon atoms of the polylactide and $\mathrm{C}_{60}$ moieties are highlighted in green and orange, respectively. 


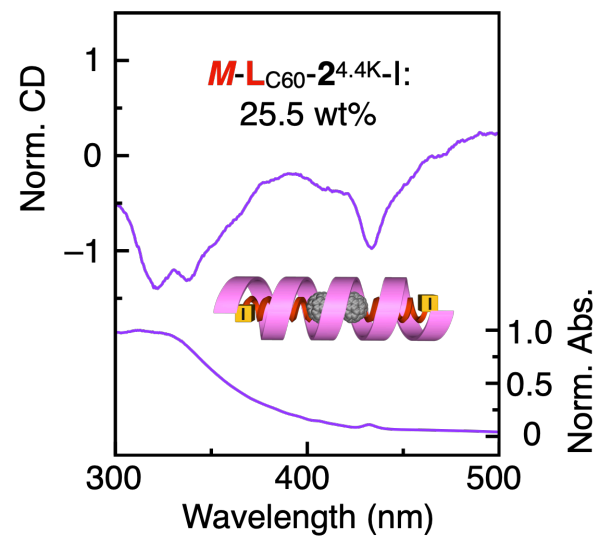

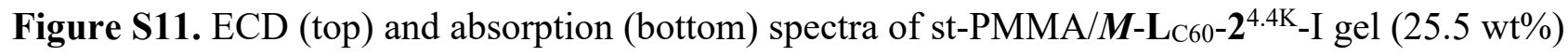
in toluene at $25^{\circ} \mathrm{C}$. The contribution of the linear dichroism caused by the macroscopic anisotropy was negligible.

(a) st-PMMA with M-L $660-\mathbf{2}^{4.4 K-I}(15.6$ wt\%)

(b) st-PMMA with $M-L_{N 3}-14.0 K-I$ (12.6 wt\%)

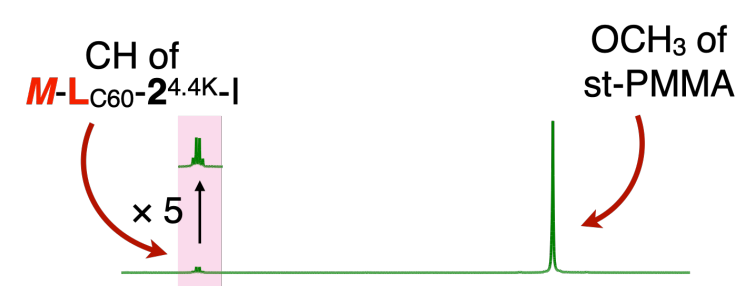

Isolated st-PMMA with $\mathrm{C}_{60}$

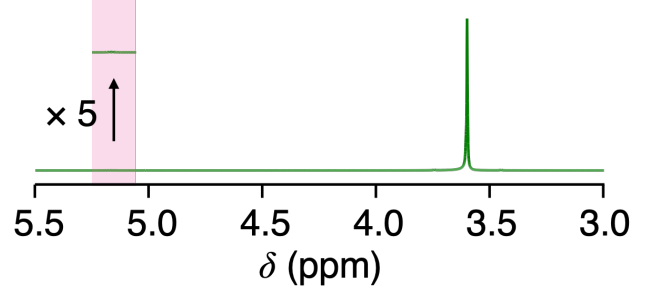

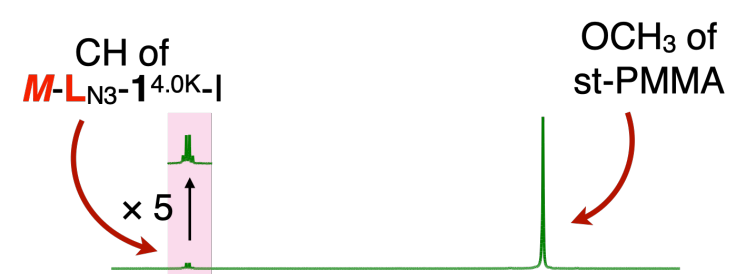

Isolated st-PMMA with $\mathrm{C}_{60}$

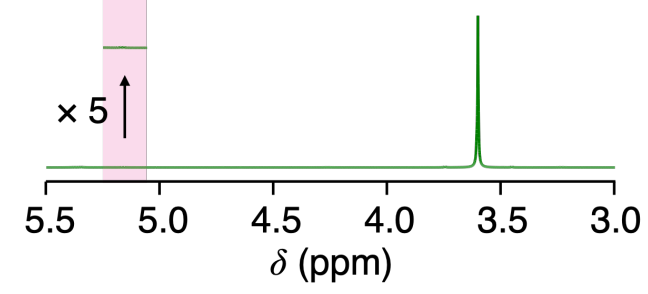

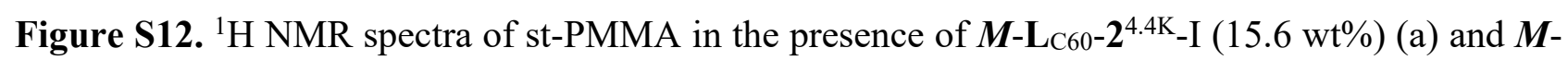
$\mathbf{L}_{\mathrm{N} 3}-\mathbf{1}^{4.0 \mathrm{~K}_{-} \mathrm{I}}\left(12.6 \mathrm{wt} \%\right.$ ) (b) (top) and those of the isolated helicity-memorized st-PMMA with $\mathrm{C}_{60}$ (bottom) in $\mathrm{CDCl}_{3}$ at $25^{\circ} \mathrm{C}$. 
(a)

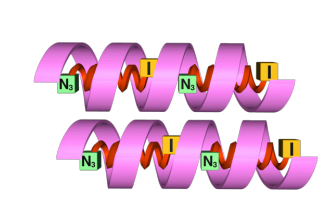

M-st-PMMA/M-LN3-14.0K-I

complex gel

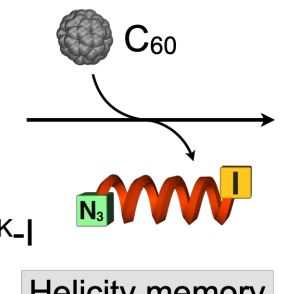

Helicity memory

in toluene at r.t.

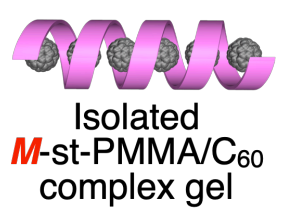

Isolated st-PMMA/C 60 complex gel prepared by the complexation with $\mathrm{C}_{60}$ after the gelation of st-PMMA with
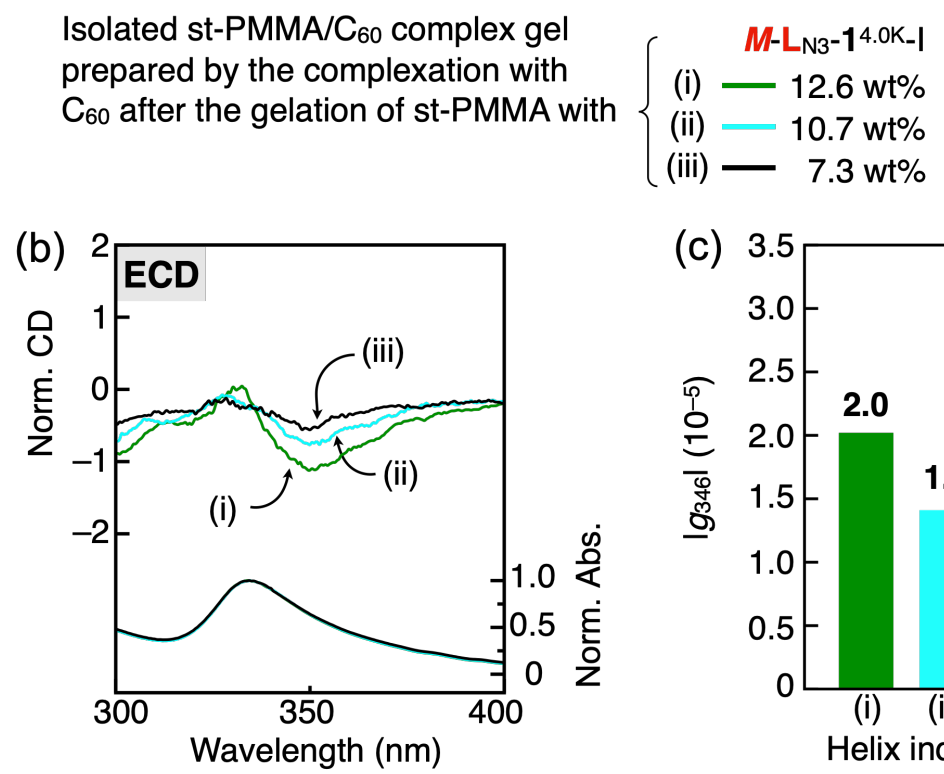

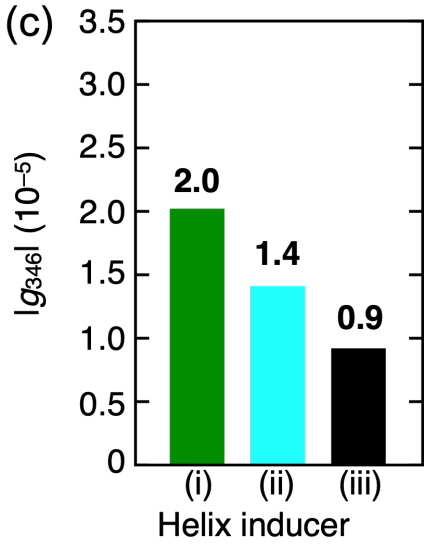

Figure S13. (a) Schematic illustration of memory of the macromolecular helicity in st-PMMA induced by inclusion complexation with $\boldsymbol{M}-\mathbf{L}_{\mathrm{N} 3}-\mathbf{1}^{4.0 \mathrm{~K}_{-}}$- followed by replacement with the achiral $\mathrm{C}_{60}$. (b) ECD (top) and absorption (bottom) spectra of isolated st-PMMA/ $\mathrm{C}_{60}$ complex gels in toluene prepared by complexation with $\boldsymbol{M}-\mathbf{L}_{\mathrm{N} 3}-\mathbf{1}^{4.0 \mathrm{~K}}-\mathrm{I}$ (i; $12.6 \mathrm{wt} \%$, ii; $10.7 \mathrm{wt} \%$, iii; $7.3 \mathrm{wt} \%$ ) followed by

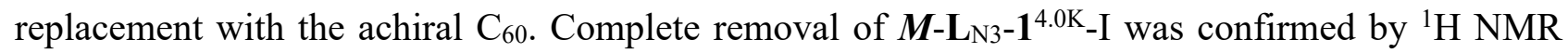
measurements of the isolated st-PMMA/C 60 complexes. The ECD and absorption spectra were normalized based on the corresponding absorption spectra at $25^{\circ} \mathrm{C}$. (c) Kuhn's dissymmetry factors at $346 \mathrm{~nm}\left(\left|g_{346}\right|\right)$ of the isolated st-PMMA/ $\mathrm{C}_{60}$ complexes (i-iii) in (b). 


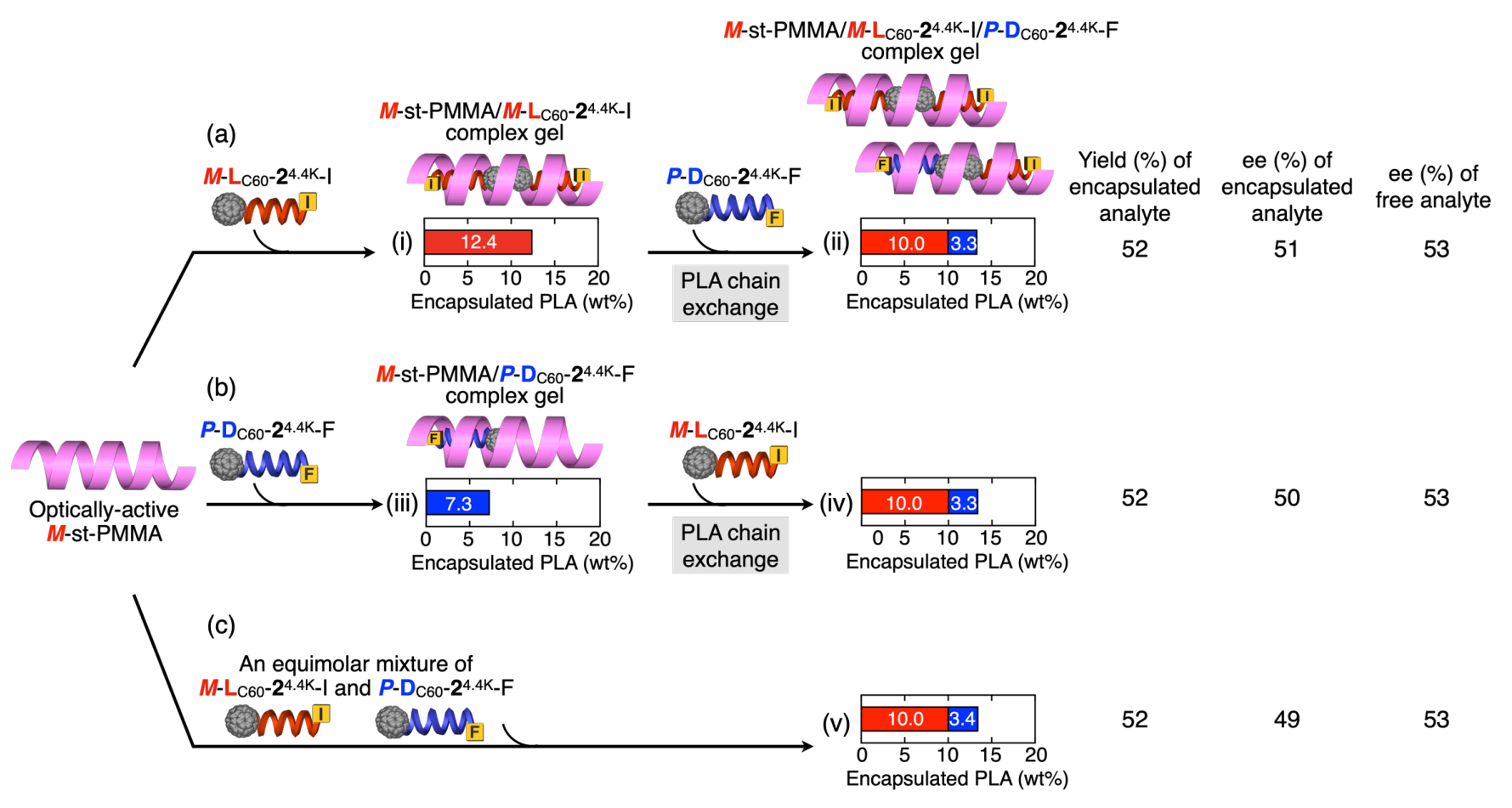

Figure S14. Schematic illustrations of the PLA chain exchange reactions between the encapsulated PLAs and free PLAs. (a) Changes in the encapsulated $\boldsymbol{M}-\mathbf{L}_{\mathrm{C} 60}-\mathbf{2}^{4.4 \mathrm{~K}_{-} \mathrm{I}}$ in the helical cavity of $M$-stPMMA before (left, i) and after adding an equimolar amount of $\boldsymbol{P}-\mathbf{D}_{\mathrm{C} 60}-\mathbf{2}^{4.4 \mathrm{~K}}-\mathrm{F}$ with stirring at room

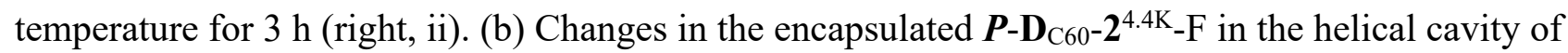

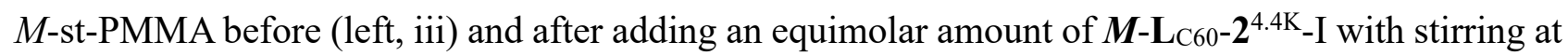
room temperature for $3 \mathrm{~h}$ (right, iv). (c) Encapsulation of an equal amount of $\boldsymbol{M}$-L-C60-2 $\mathbf{2}^{4.4 \mathrm{~K}_{-}}$I and $\boldsymbol{P}$ -

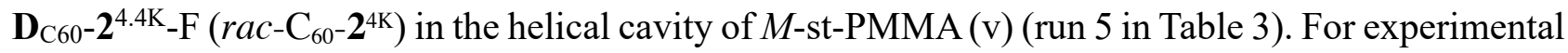
conditions, see Sections 7 and 8. The amounts of the encapsulated PLAs in $M$-st-PMMA were estimated by absorption (i, iii) and ${ }^{1} \mathrm{H}$ NMR (ii, iv, v) analyses. The molar ratios of the encapsulated nonracemic PLAs in $M$-st-PMMA (ii, iv, v) were estimated by ${ }^{1} \mathrm{H}$ NMR analyses.

The results clearly indicated that the PLA chain exchange reactions proceeded to reach an equilibrium within $3 \mathrm{~h}$ at room temperature. 


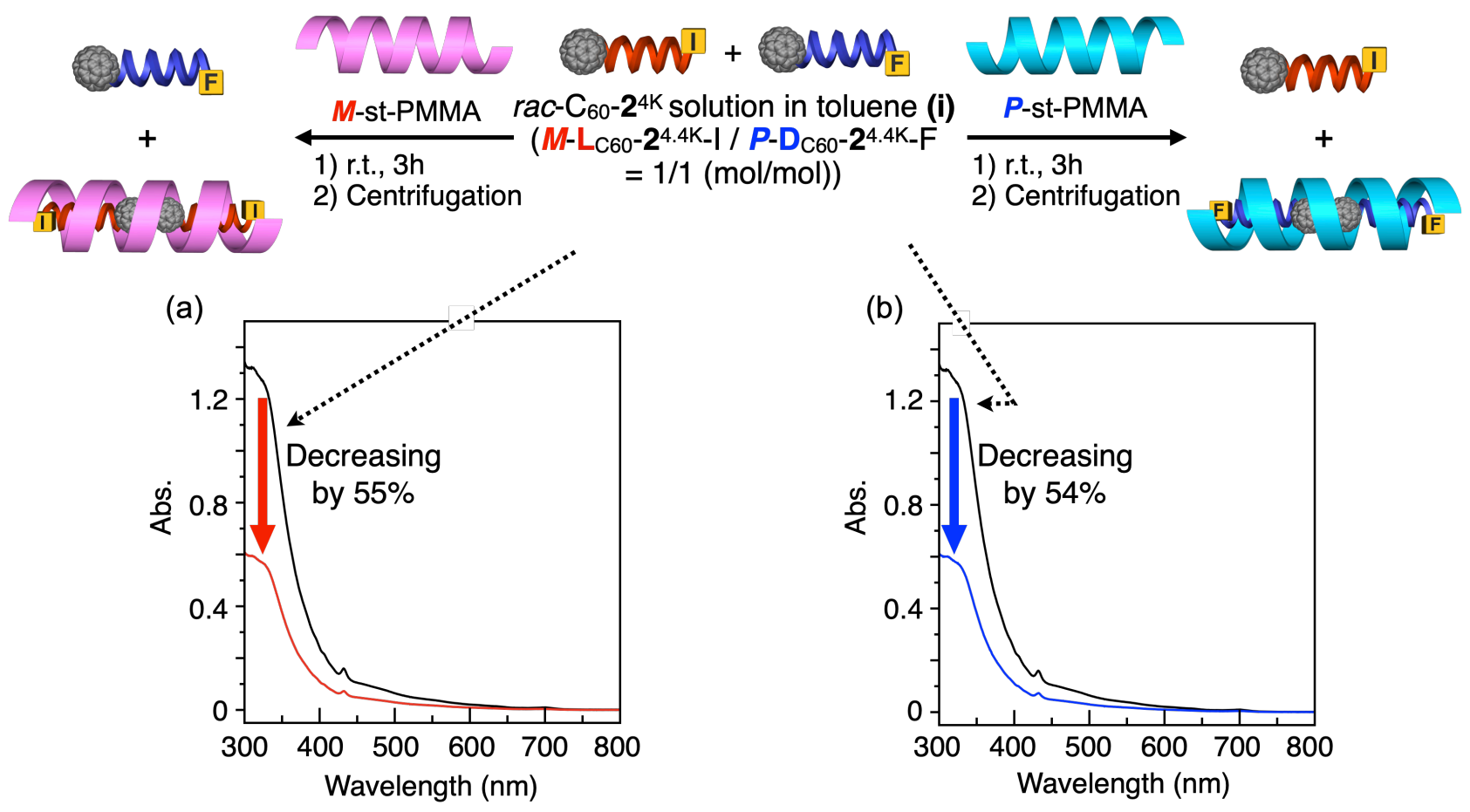

Figure S15. (a, b) Absorption spectra of toluene solutions of the feed $r a c-\mathrm{C}_{60}-2^{4 \mathrm{~K}}$ (an equimolar

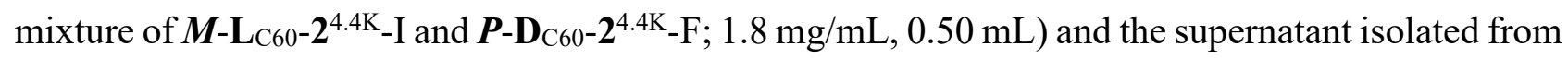
the st-PMMA/nonracemic $\mathrm{C}_{60-\mathbf{2}}{ }^{\mathrm{K}}$ complex gels after the addition of $\boldsymbol{M}$-st-PMMA (a) and $\boldsymbol{P}$-stPMMA (b) $(3.0 \mathrm{mg}$ ) with subsequent stirring at r.t. for $3 \mathrm{~h}$ and then centrifuged at $1700 \mathrm{~g}$ for $10 \mathrm{~min}$. 


\section{Supporting References}

(S1) Abe, H.; Imai, K.; Matsumoto, M. Syndiotactic Polymerization of Methyl Methacrylate. J. Polym. Sci., Part C 1968, 23, 469-485.

(S2) Maggini, M.; Karlsson, A.; Pasimeni, L.; Scorrano, G.; Prato, M.; Valli, L. Synthesis of NAcylated Fulleropyrrolidines: New Materials for the Preparation of Langmuir-Blodgett Films Containing Fullerenes. Tetrahedron Lett. 1994, 35, 2985-2988.

(S3) Isono, T.; Kondo, Y.; Otsuka, I.; Nishiyama, Y.; Borsali, R.; Kakuchi, T.; Satoh, T. Synthesis and Stereocomplex Formation of Star-Shaped Stereoblock Polylactides Consisting of Poly(l-lactide) and Poly(d-lactide) Arms. Macromolecules 2013, 46, 8509-8518.

(S4) Ousaka, N.; Mamiya, F.; Iwata, Y.; Nishimura, K.; Yashima, E. "Helix-in-Helix" Superstructure Formation through Encapsulation of Fullerene-Bound Helical Peptides within a Helical Poly(methyl methacrylate) Cavity. Angew. Chem., Int. Ed. 2017, 56, 791-795.

(S5) Harrell, M. L.; Bergbreiter, D. E. Using ${ }^{1} \mathrm{H}$ NMR Spectra of Polymers and Polymer Products to Illustrate Concepts in Organic Chemistry. J. Chem. Educ. 2017, 94, 1668-1673.

(S6) Hoogsteen, W.; Postema, A. R.; Pennings, A. J.; Tenbrinke, G.; Zugenmaier, P. Crystal Structure, Conformation, and Morphology of Solution-Spun Poly(L-lactide) Fibers. Macromolecules 1990, $23,634-642$.

(S7) Alemán, C.; Lotz, B.; Puiggali, J. Crystal Structure of the $\alpha$-Form of Poly(L-lactide). Macromolecules 2001, 34, 4795-4801.

(S8) Sun, H.; Jin, Z.; Yang, C.; Akkermans, R. L. C.; Robertson, S. H.; Spenley, N. A.; Miller, S.; Todd, S. M. COMPASS II: Extended Coverage for Polymer and Drug-like Molecule Databases. J. Mol. Model. 2016, 22, 47.

(S9) Kumaki, J.; Kawauchi, T.; Okoshi, K.; Kusanagi, H.; Yashima, E. Supramolecular Helical Structure of the Stereocomplex Composed of Complementary Isotactic and Syndiotactic Poly(methyl methacrylate)s as Revealed by Atomic Force Microscopy. Angew. Chem., Int. Ed. 2007, 46, 5348-5351.

(S10) Kawauchi, T.; Kitaura, A.; Kawauchi, M.; Takeichi, T.; Kumaki, J.; Iida, H.; Yashima, E. Separation of $\mathrm{C}_{70}$ over $\mathrm{C}_{60}$ and Selective Extraction and Resolution of Higher Fullerenes by Syndiotactic Helical Poly(methyl methacrylate). J. Am. Chem. Soc. 2010, 132, 12191-12193.

(S11) Kitaura, A.; Iida, H.; Kawauchi, T.; Yashima, E. Helicity Induction and Memory of Syndiotactic Poly(methyl methacrylate) Assisted by Optically Active Additives and Solvents and Chiral Amplification of Helicity. Chem. Lett. 2011, 40, 28-30. 


\section{2. ${ }^{1} \mathrm{H}$ and ${ }^{13} \mathrm{C}$ NMR Spectral Data}

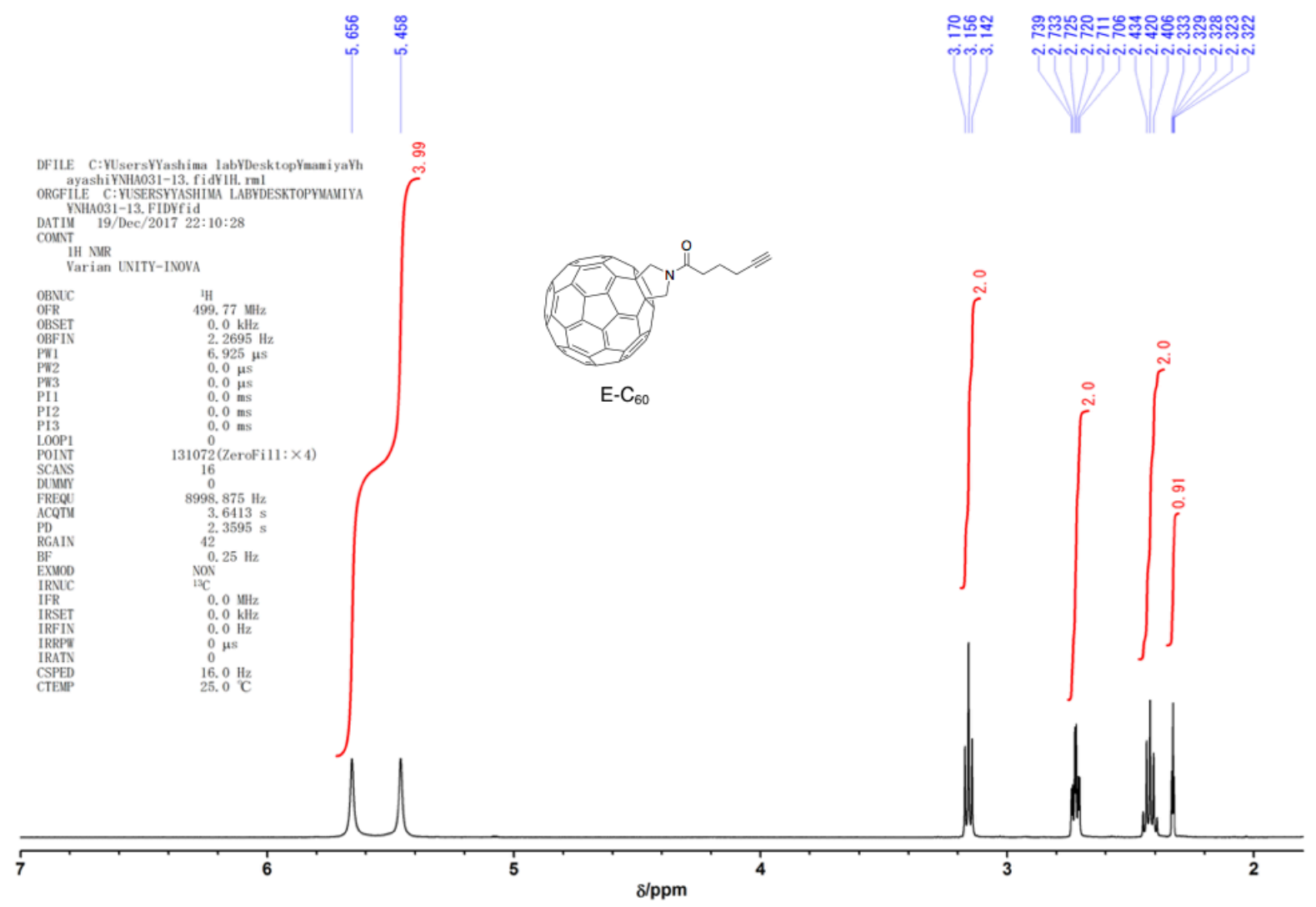

Figure S16. ${ }^{1} \mathrm{H}$ NMR $(500 \mathrm{MHz})$ spectrum of E-C 60 in $o$-dichlorobenzene- $d_{4}$ at $25^{\circ} \mathrm{C}$.

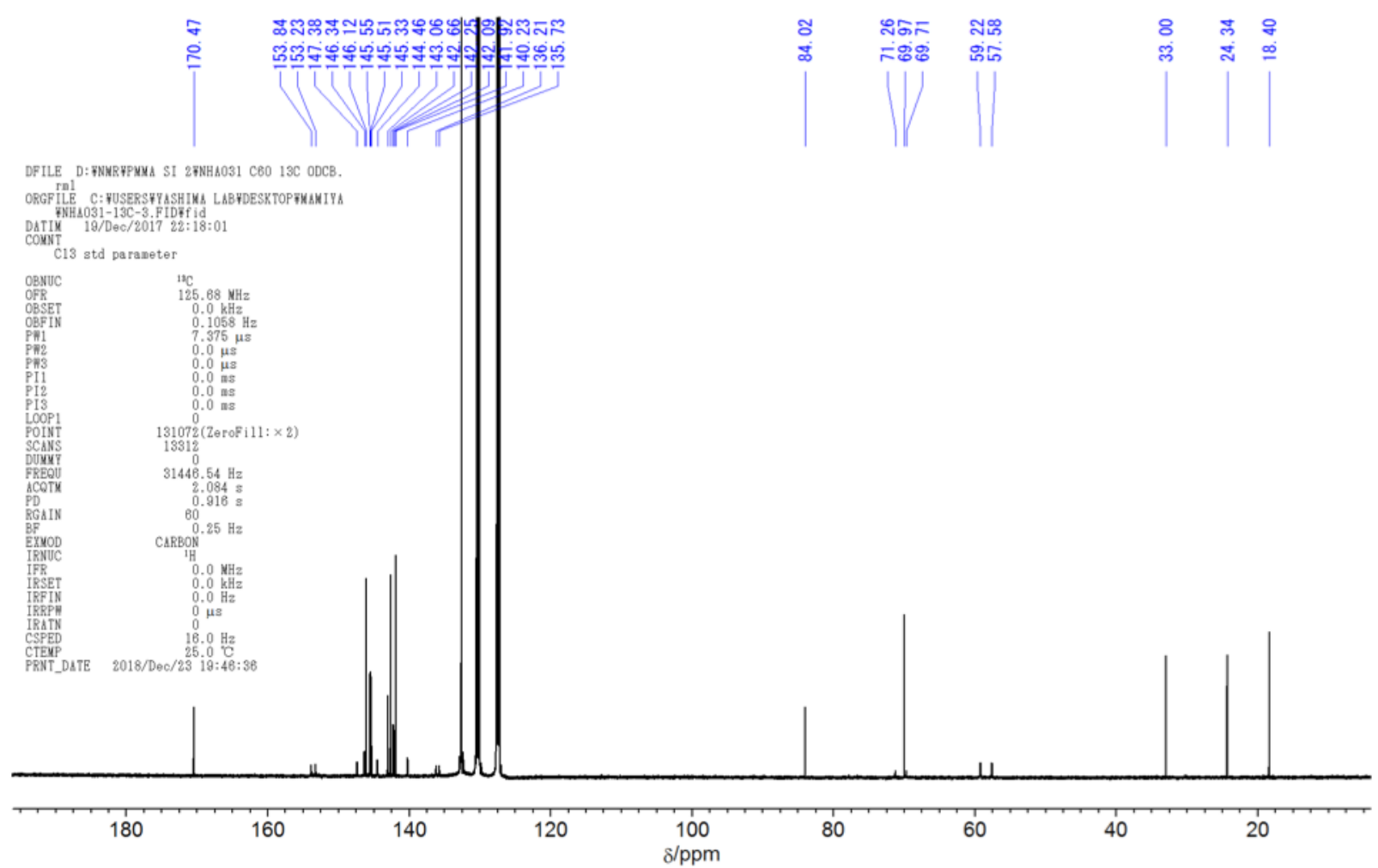

Figure S17. ${ }^{13} \mathrm{C}$ NMR $(126 \mathrm{MHz})$ spectrum of E-C 60 in $o$-dichlorobenzene- $d_{4}$ at $25{ }^{\circ} \mathrm{C}$. 


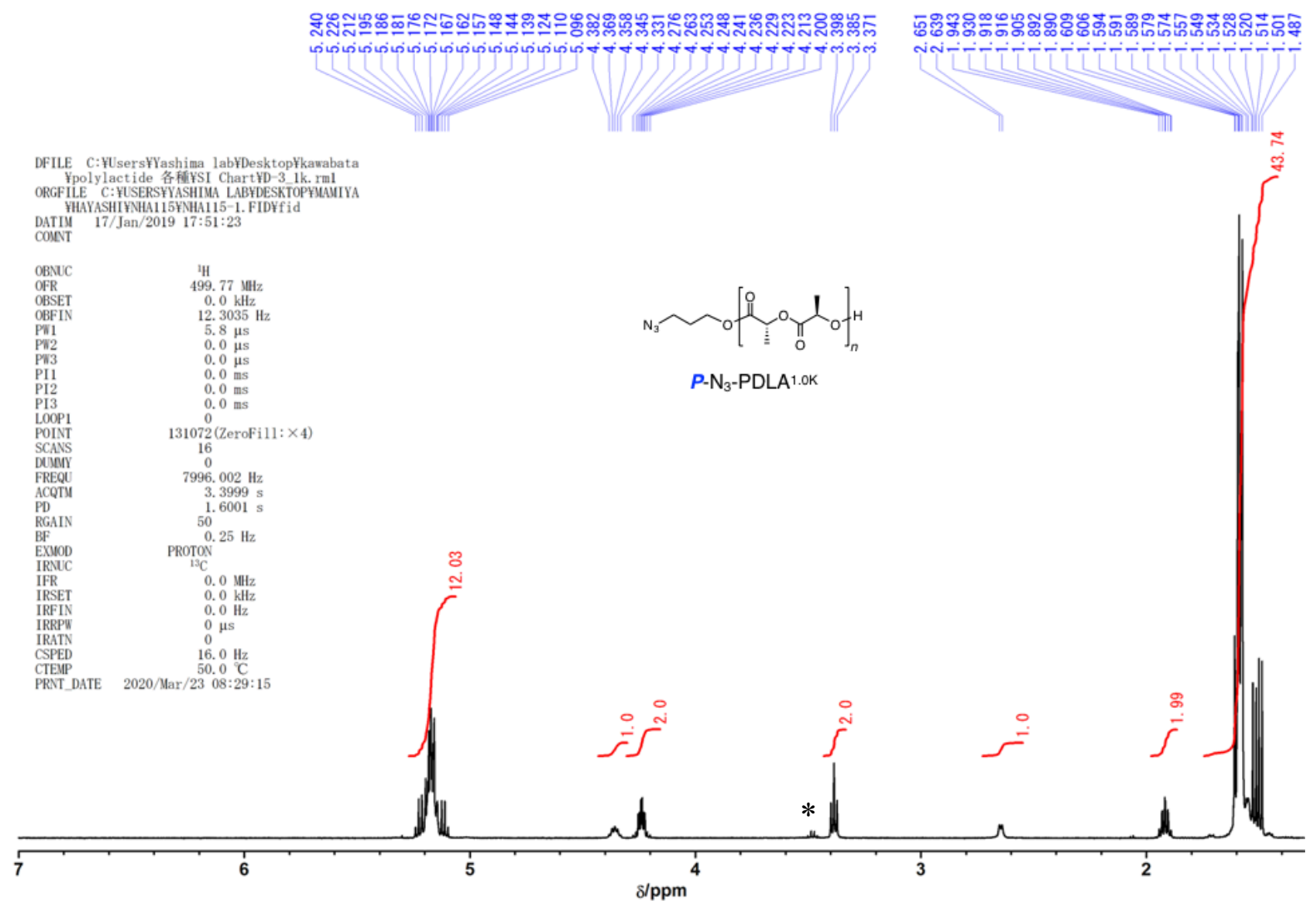

Figure S18. ${ }^{1} \mathrm{H}$ NMR $(500 \mathrm{MHz})$ spectrum of $\boldsymbol{P}-\mathrm{N}_{3}-\mathrm{PDLA}^{1.0 \mathrm{~K}}$ in $\mathrm{CDCl}_{3}$ at $25^{\circ} \mathrm{C} .{ }^{*}$ denote the protons from residual $\mathrm{Et}_{2} \mathrm{O}$.

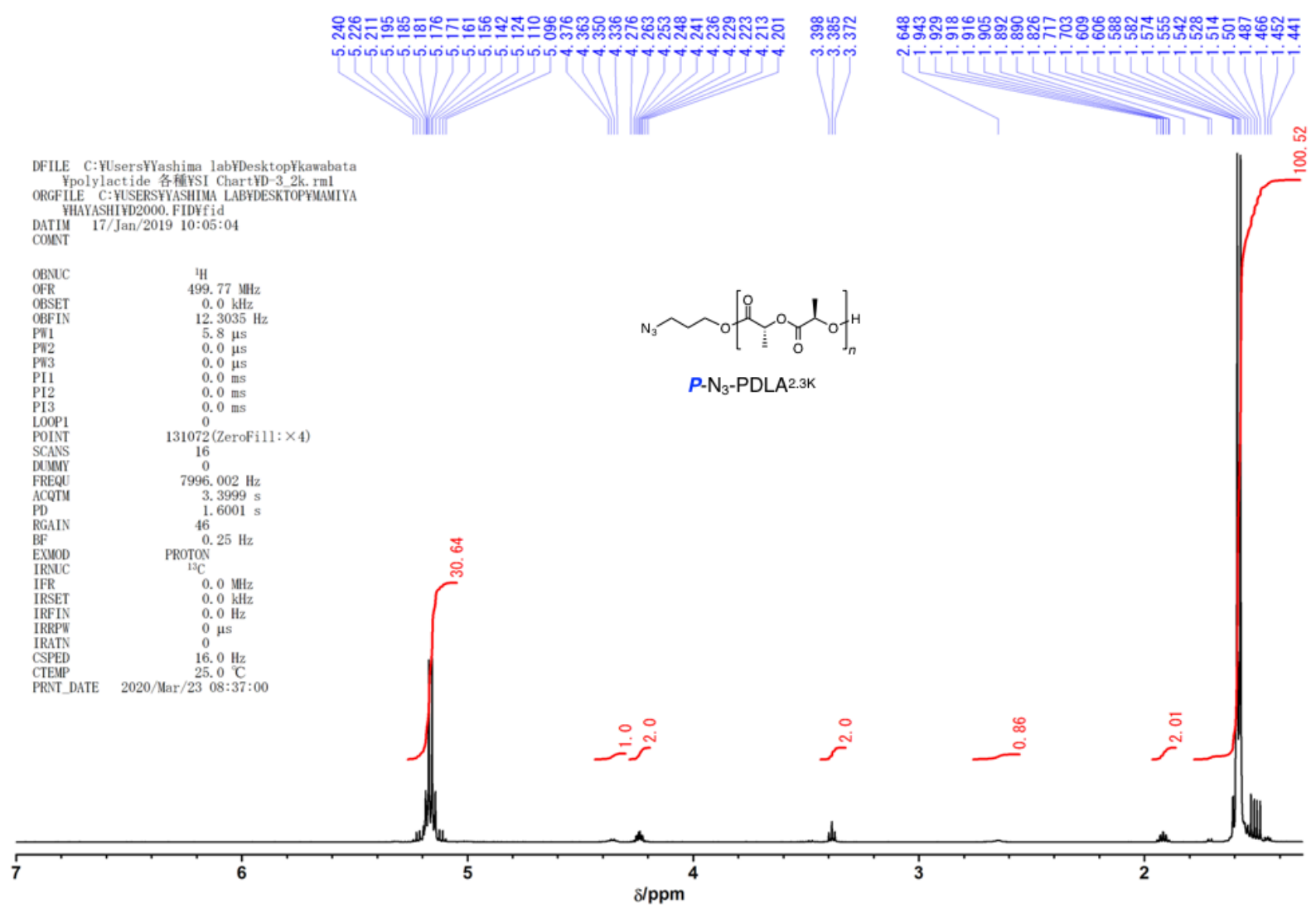

Figure S19. ${ }^{1} \mathrm{H}$ NMR $(500 \mathrm{MHz})$ spectrum of $\boldsymbol{P}-\mathrm{N}_{3}-\mathrm{PDLA}^{2.3 \mathrm{~K}}$ in $\mathrm{CDCl}_{3}$ at $25{ }^{\circ} \mathrm{C}$. 


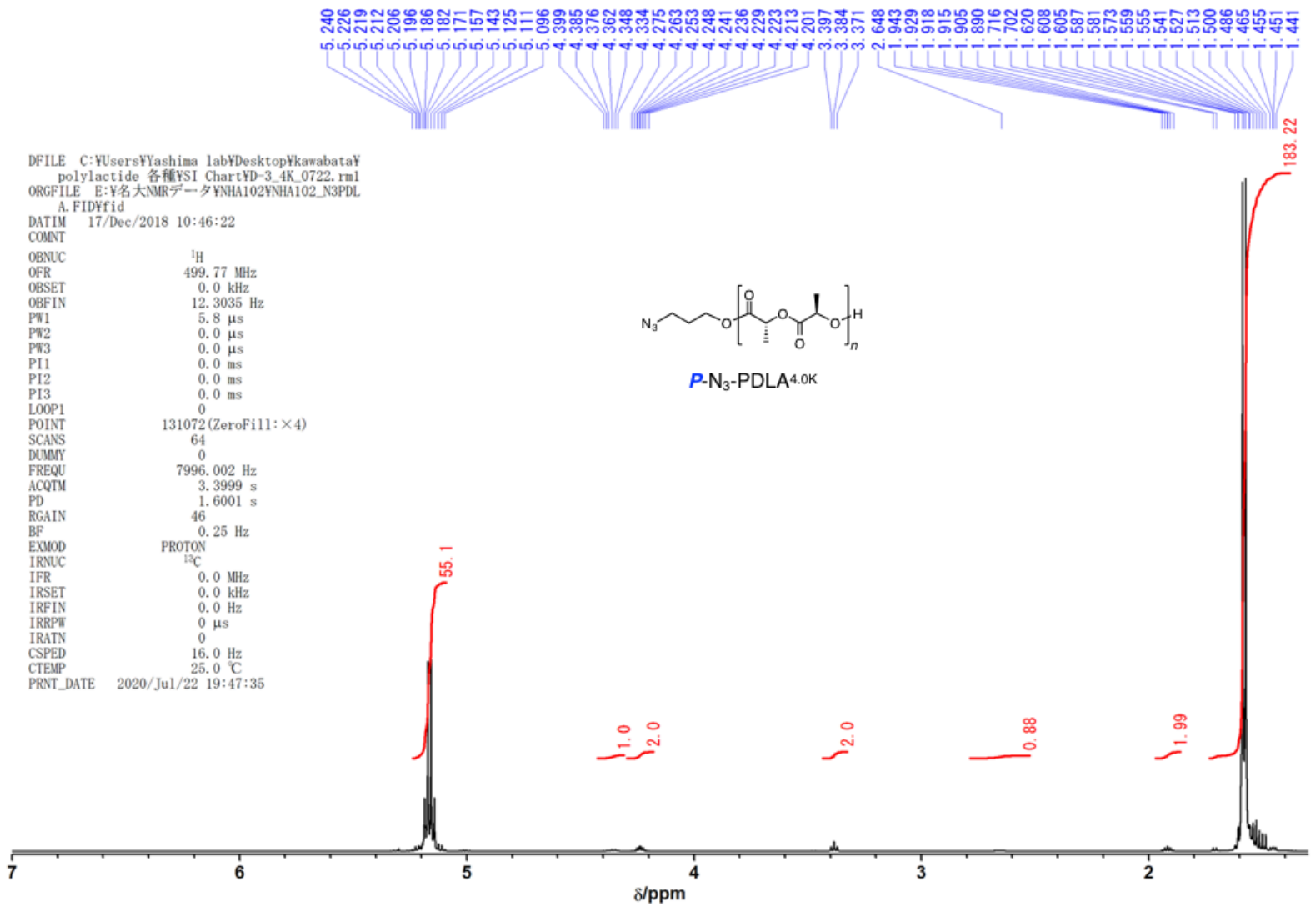

Figure S20. ${ }^{1} \mathrm{H}$ NMR $(500 \mathrm{MHz})$ spectrum of $\boldsymbol{P}-\mathrm{N}_{3}-\mathrm{PDLA}^{4.0 \mathrm{~K}}$ in $\mathrm{CDCl}_{3}$ at $25{ }^{\circ} \mathrm{C}$.

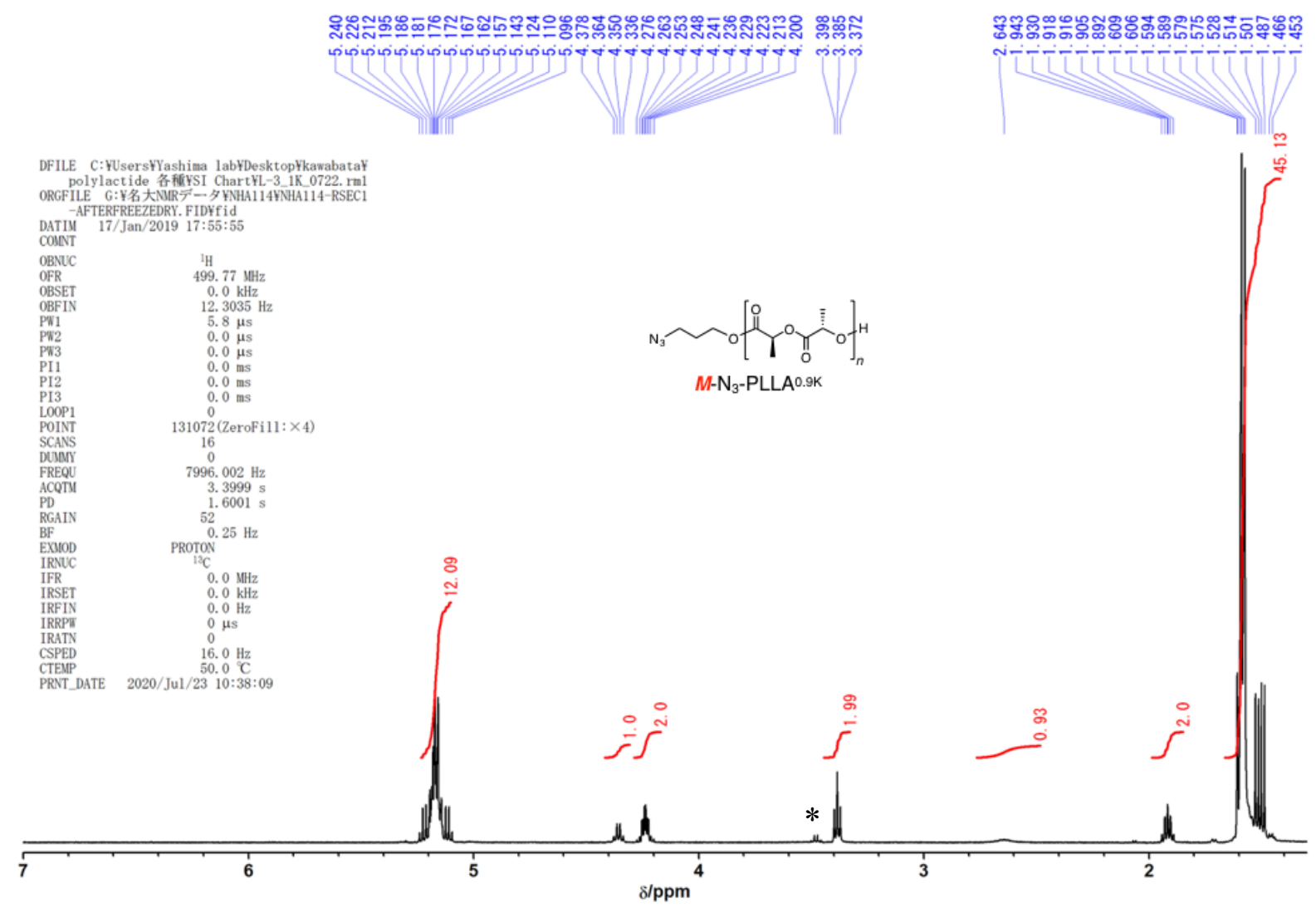

Figure S21. ${ }^{1} \mathrm{H}$ NMR $(500 \mathrm{MHz})$ spectrum of $\boldsymbol{M}-\mathrm{N}_{3}-\mathrm{PLLA}^{0.9 \mathrm{~K}}$ in $\mathrm{CDCl}_{3}$ at $25{ }^{\circ} \mathrm{C}$. * denotes the protons from residual $\mathrm{Et}_{2} \mathrm{O}$. 


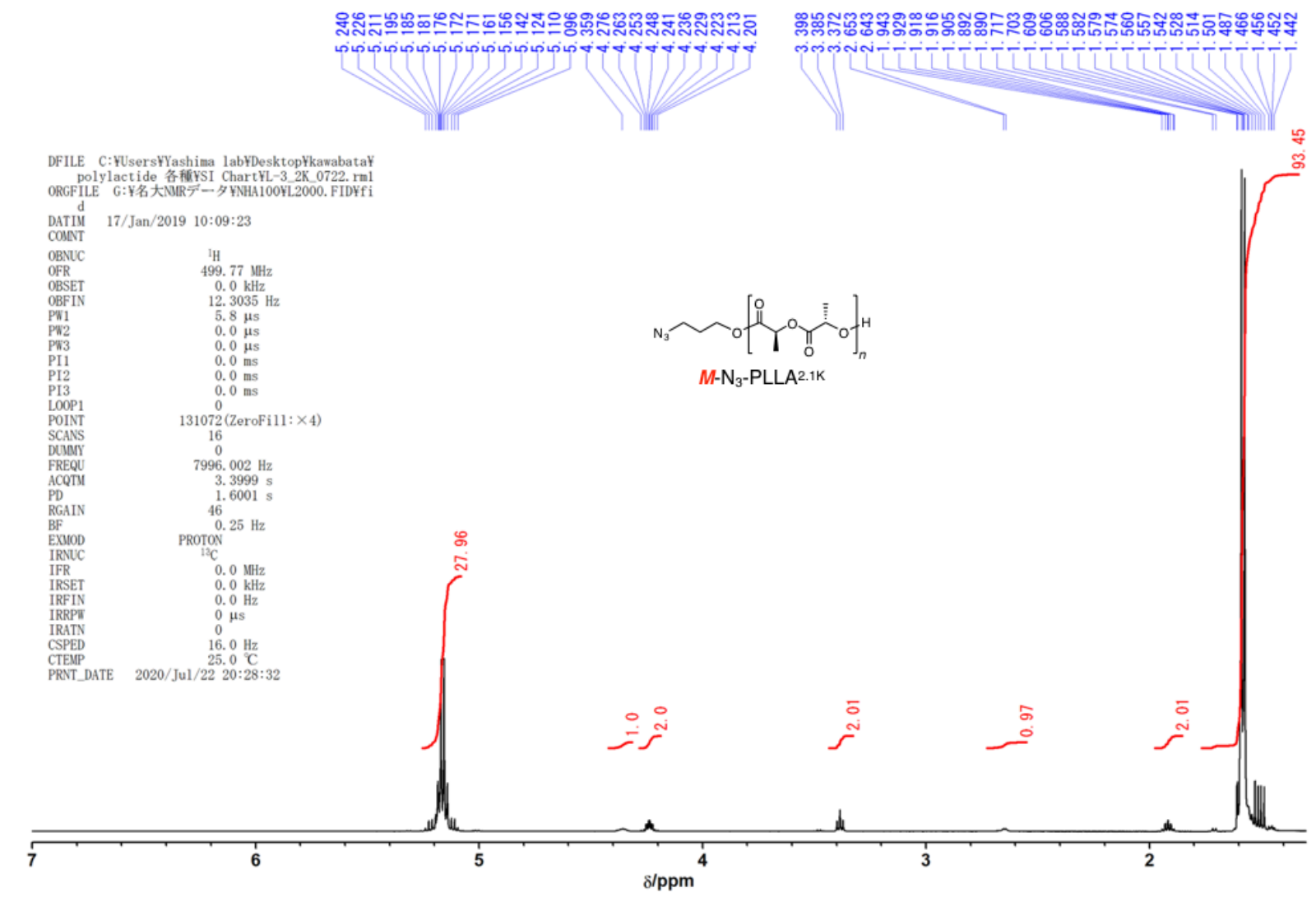

Figure S22. ${ }^{1} \mathrm{H}$ NMR $(500 \mathrm{MHz})$ spectrum of $\boldsymbol{M}$ - $\mathrm{N}_{3}-\mathrm{PLLA}^{2.1 \mathrm{~K}}$ in $\mathrm{CDCl}_{3}$ at $25^{\circ} \mathrm{C}$.

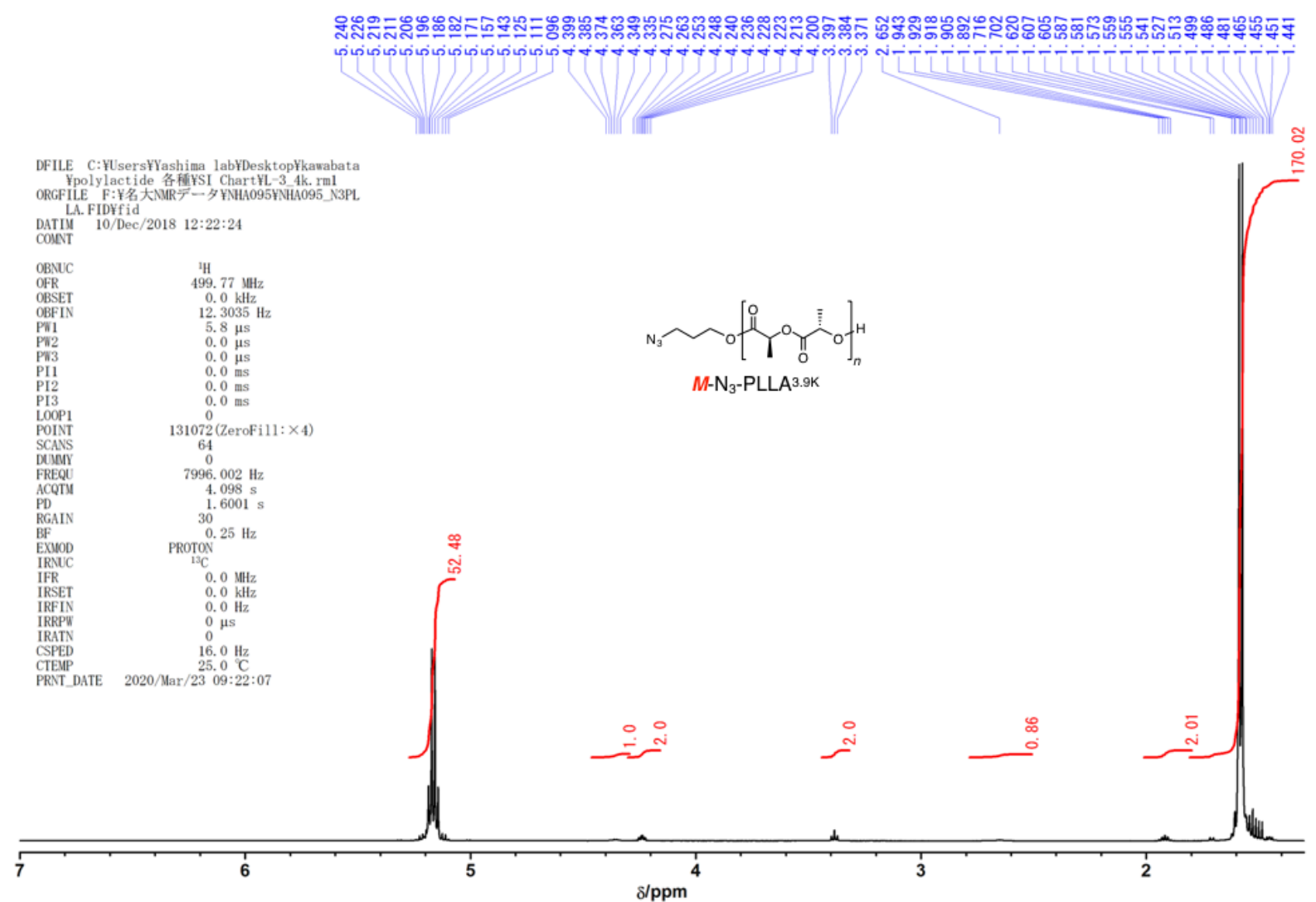

Figure S23. ${ }^{1} \mathrm{H}$ NMR $(500 \mathrm{MHz})$ spectrum of $\boldsymbol{M}$ - $\mathrm{N}_{3}-\mathrm{PLLA}^{3.9 \mathrm{~K}}$ in $\mathrm{CDCl}_{3}$ at $25^{\circ} \mathrm{C}$. 


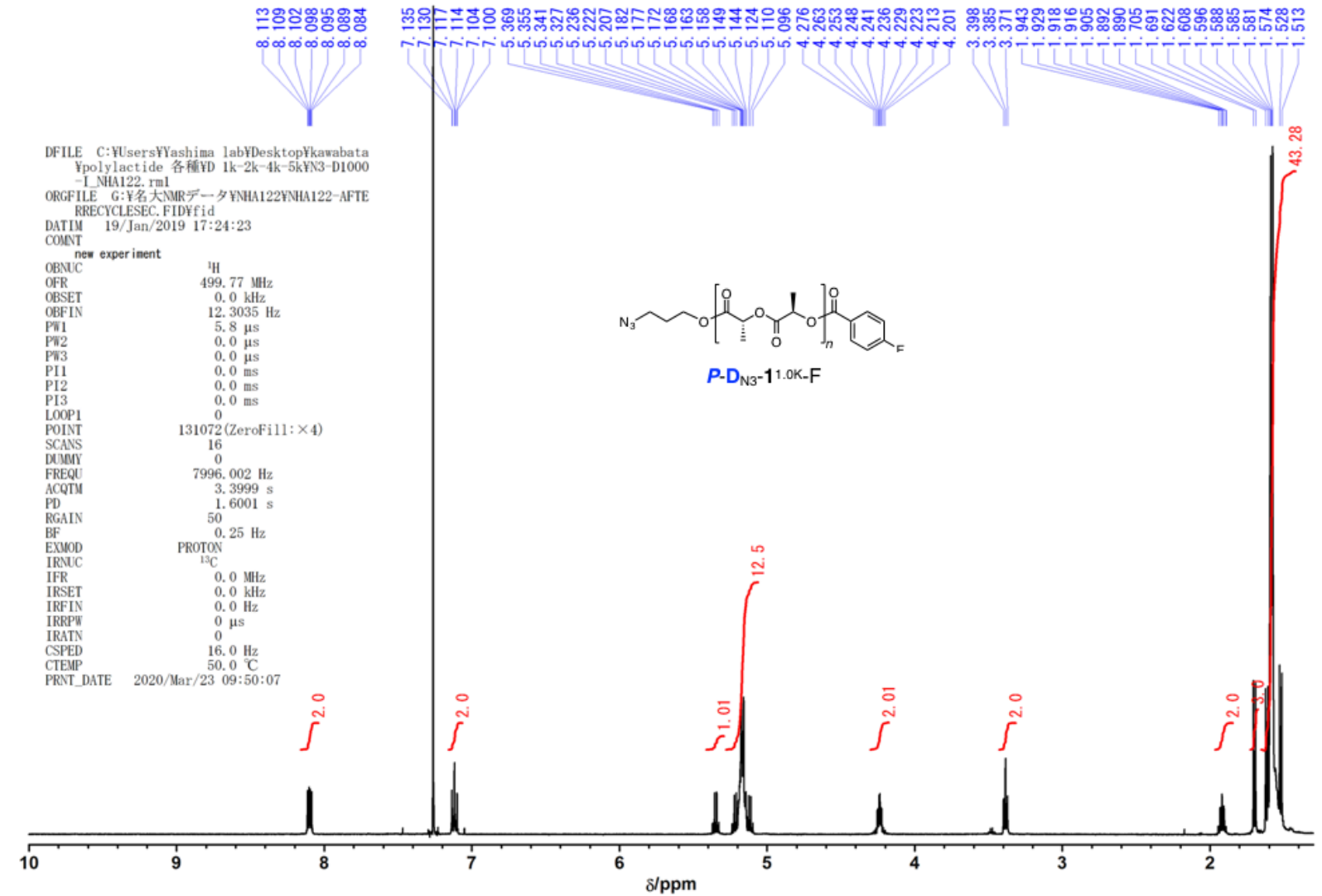

Figure S24. ${ }^{1} \mathrm{H}$ NMR $(500 \mathrm{MHz})$ spectrum of $\boldsymbol{P}-\mathbf{D}_{\mathrm{N} 3}-\mathbf{1}^{1.0 \mathrm{~K}}-\mathrm{F}$ in $\mathrm{CDCl}_{3}$ at $25^{\circ} \mathrm{C}$.

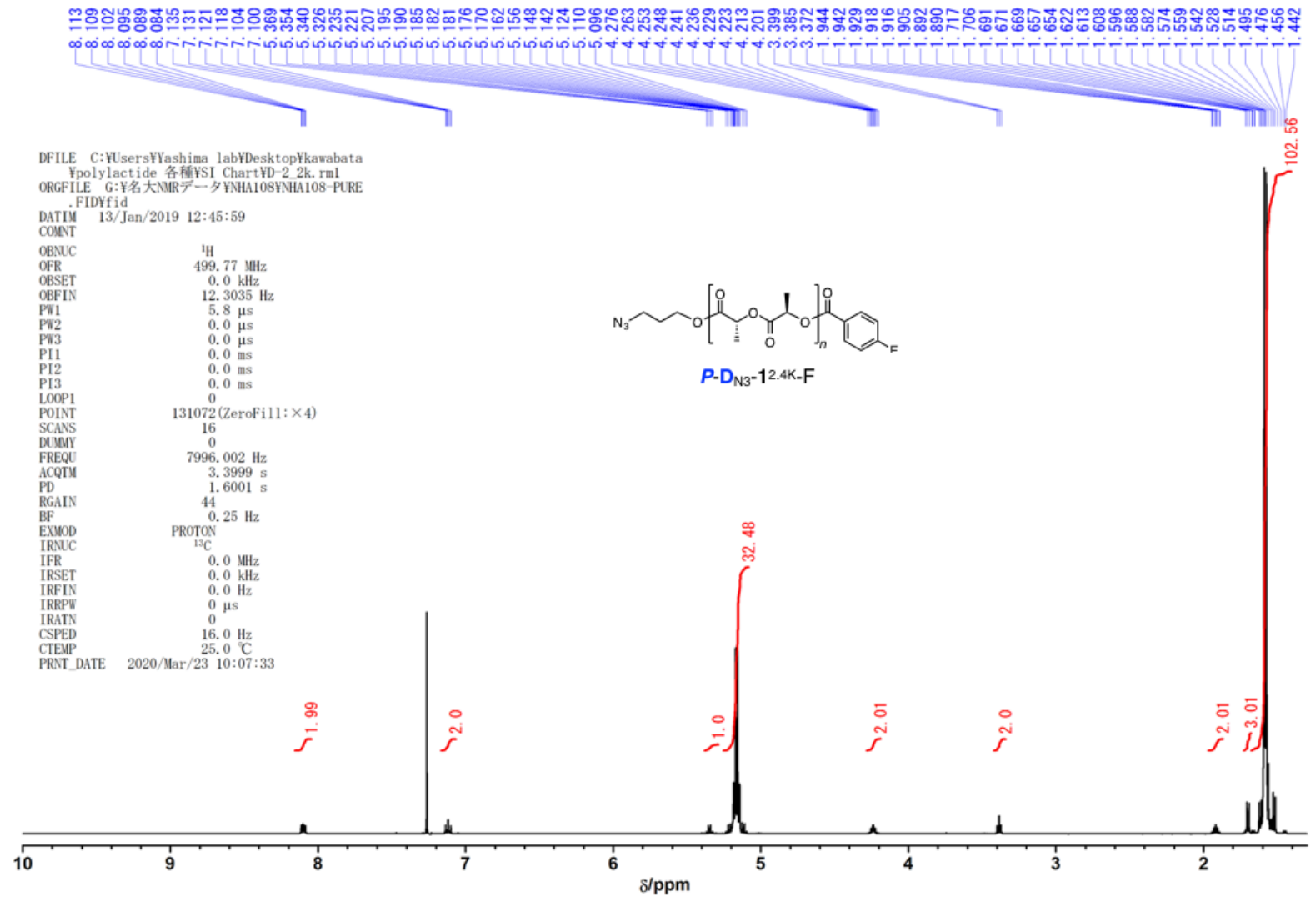

Figure S25. ${ }^{1} \mathrm{H}$ NMR $(500 \mathrm{MHz})$ spectrum of $\boldsymbol{P}-\mathbf{D}_{\mathrm{N} 3}-\mathbf{1}^{2.4 \mathrm{~K}}-\mathrm{F}$ in $\mathrm{CDCl}_{3}$ at $25{ }^{\circ} \mathrm{C}$. 


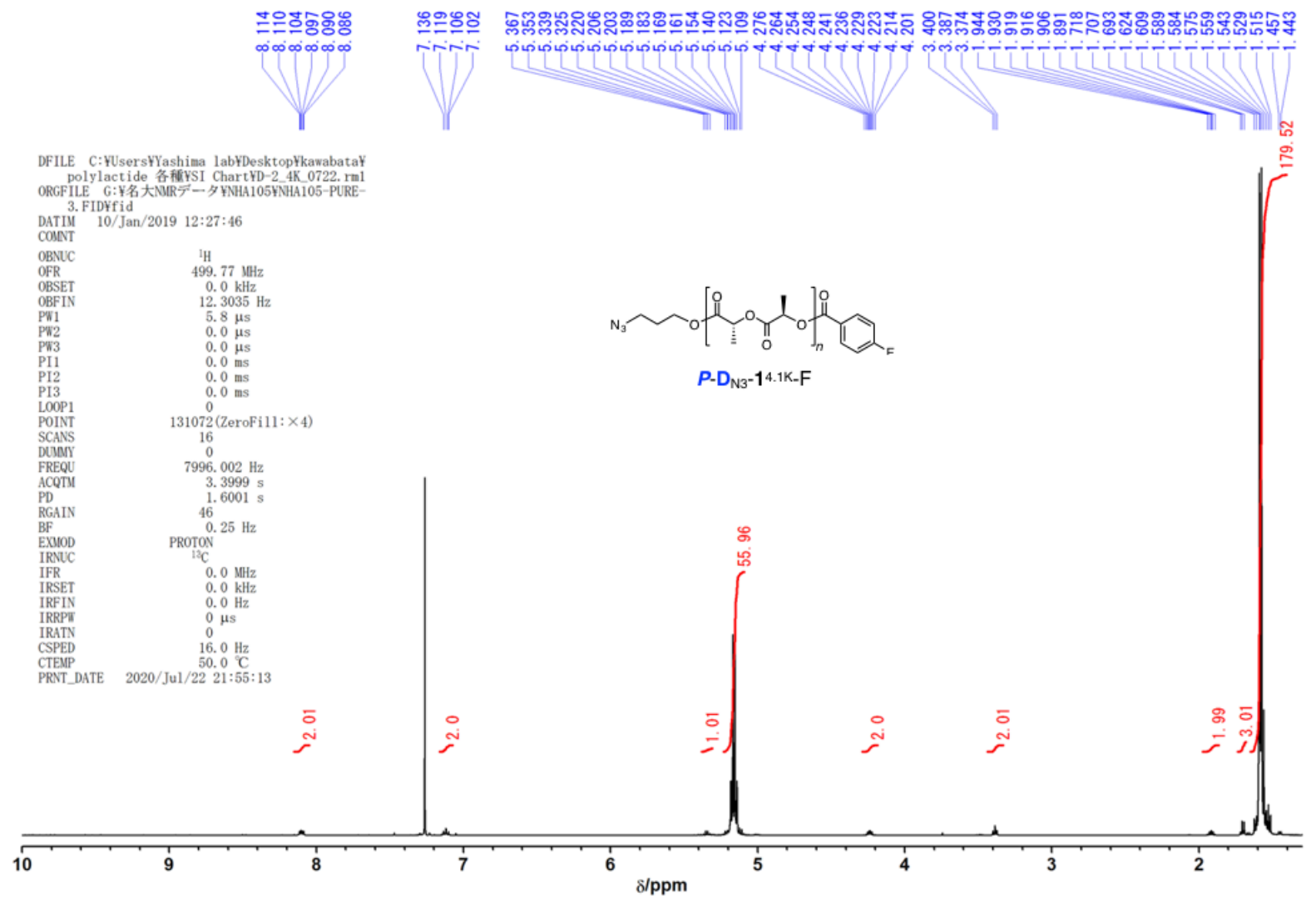

Figure S26. ${ }^{1} \mathrm{H}$ NMR $(500 \mathrm{MHz})$ spectrum of $\boldsymbol{P}-\mathbf{D}_{\mathrm{N} 3}-\mathbf{1}^{4.1 \mathrm{~K}_{-} \mathrm{F}}$ in $\mathrm{CDCl}_{3}$ at $25{ }^{\circ} \mathrm{C}$.

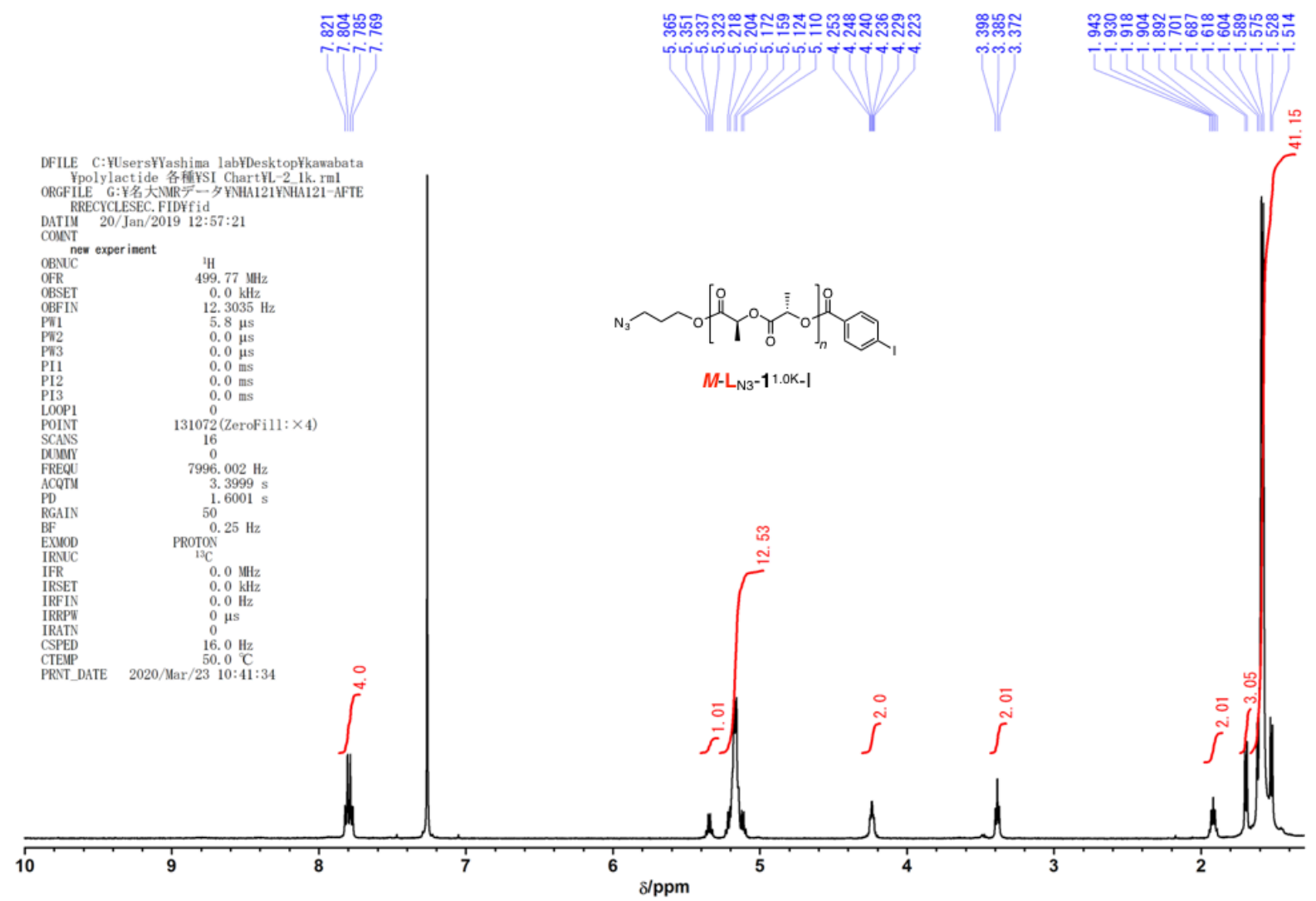

Figure S27. ${ }^{1} \mathrm{H}$ NMR $(500 \mathrm{MHz})$ spectrum of $\boldsymbol{M}-\mathbf{L}_{\mathrm{N} 3}-\mathbf{1}^{1.0 \mathrm{~K}}-\mathrm{I}$ in $\mathrm{CDCl}_{3}$ at $25^{\circ} \mathrm{C}$. 


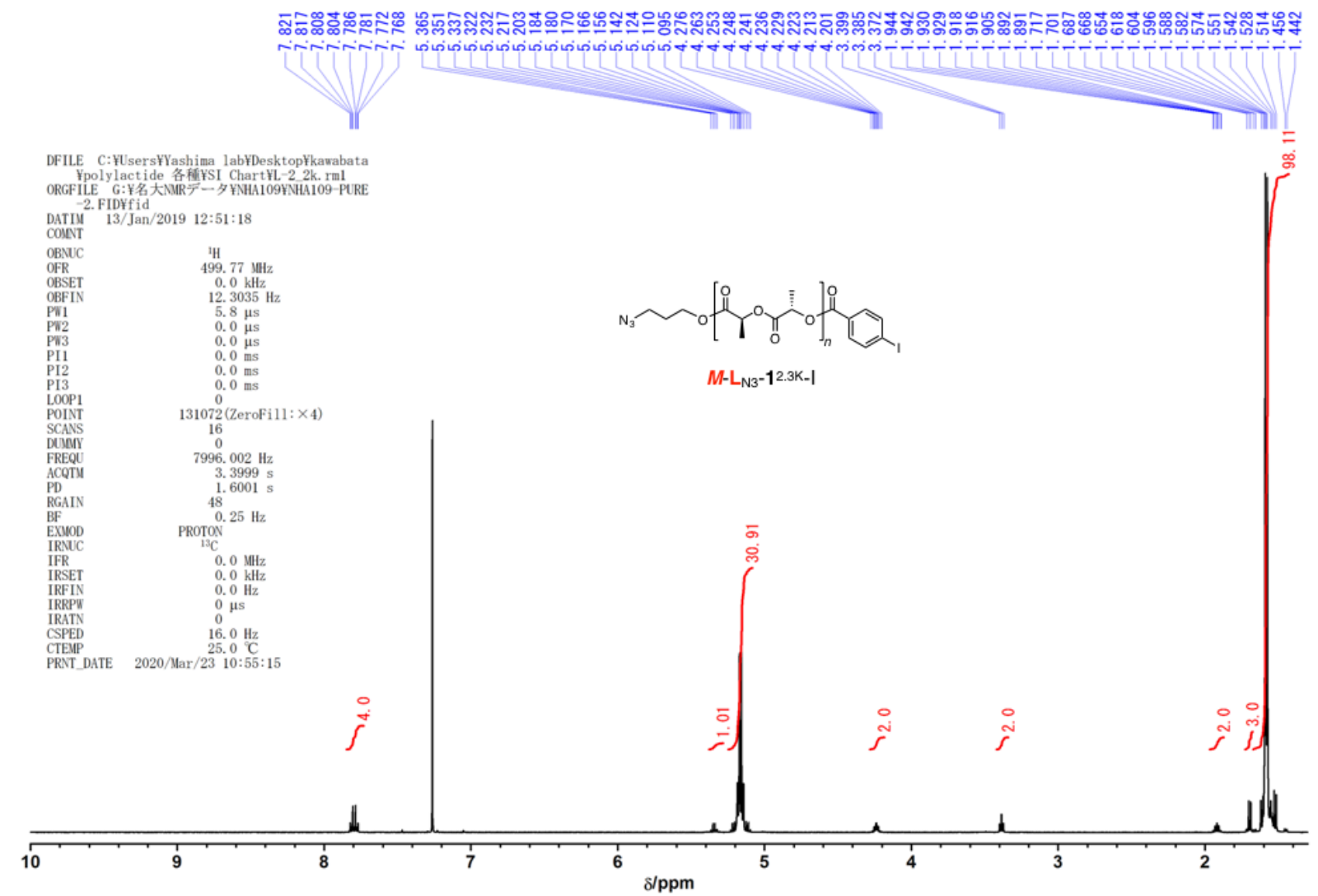

Figure S28. ${ }^{1} \mathrm{H}$ NMR $(500 \mathrm{MHz})$ spectrum of $\boldsymbol{M}-\mathbf{L}_{\mathrm{N} 3}-\mathbf{1}^{2.3 \mathrm{~K}}-\mathrm{I}$ in $\mathrm{CDCl}_{3}$ at $25^{\circ} \mathrm{C}$.

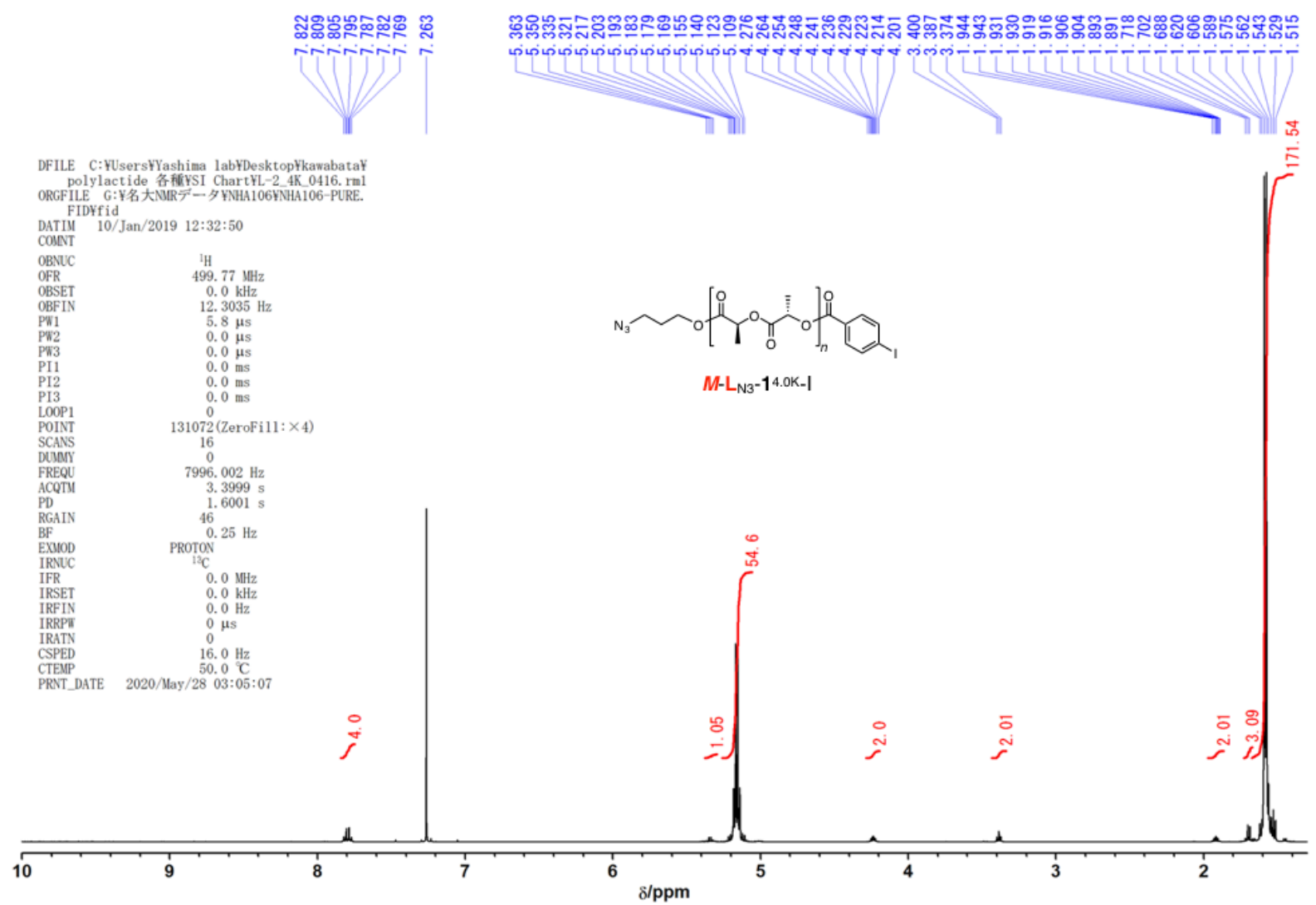

Figure S29. ${ }^{1} \mathrm{H}$ NMR $(500 \mathrm{MHz})$ spectrum of $\boldsymbol{M}-\mathbf{L}_{\mathrm{N} 3}-\mathbf{1}^{4.0 \mathrm{~K}}-\mathrm{I}$ in $\mathrm{CDCl}_{3}$ at $25{ }^{\circ} \mathrm{C}$. 


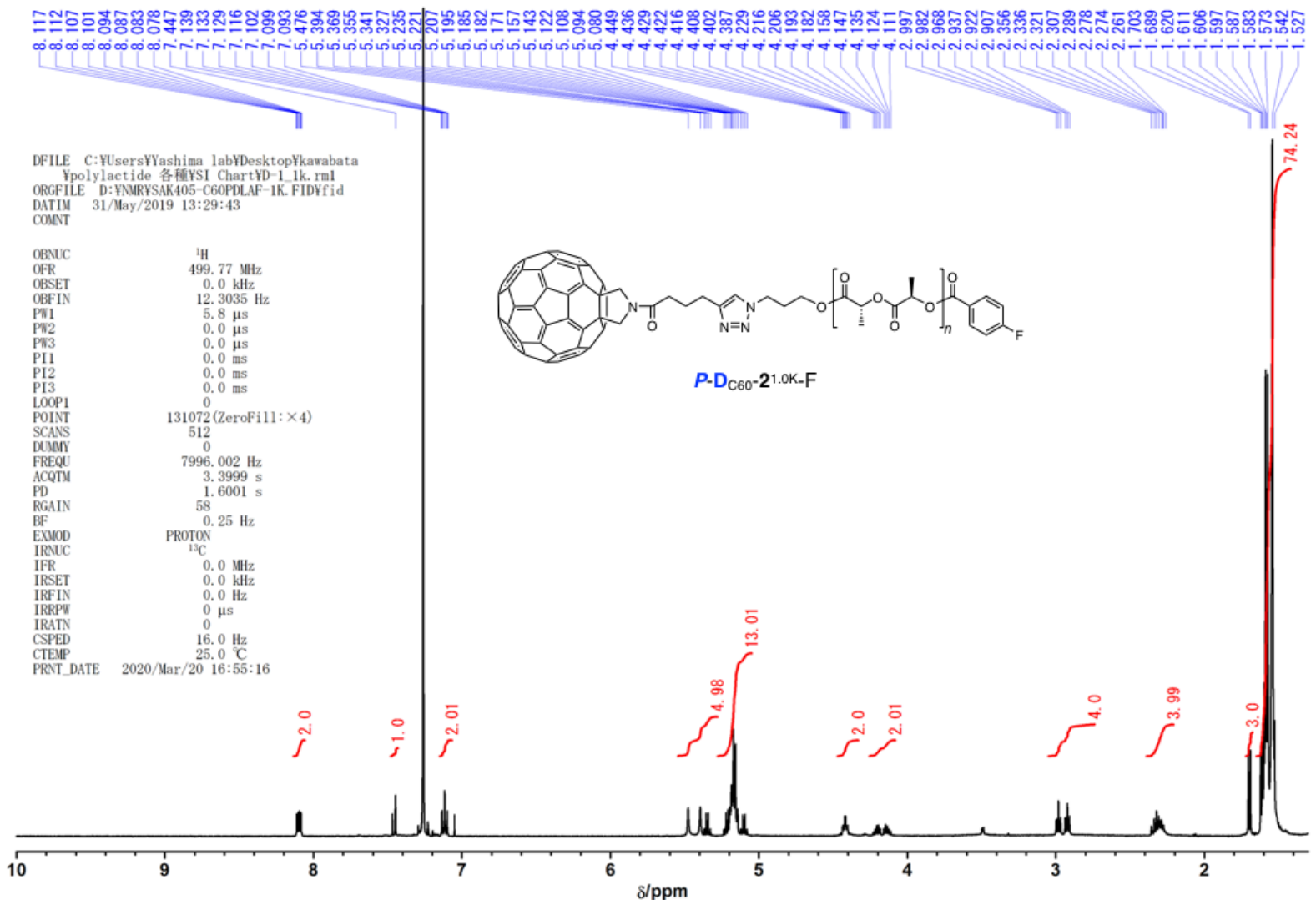

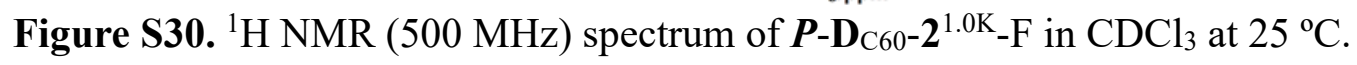

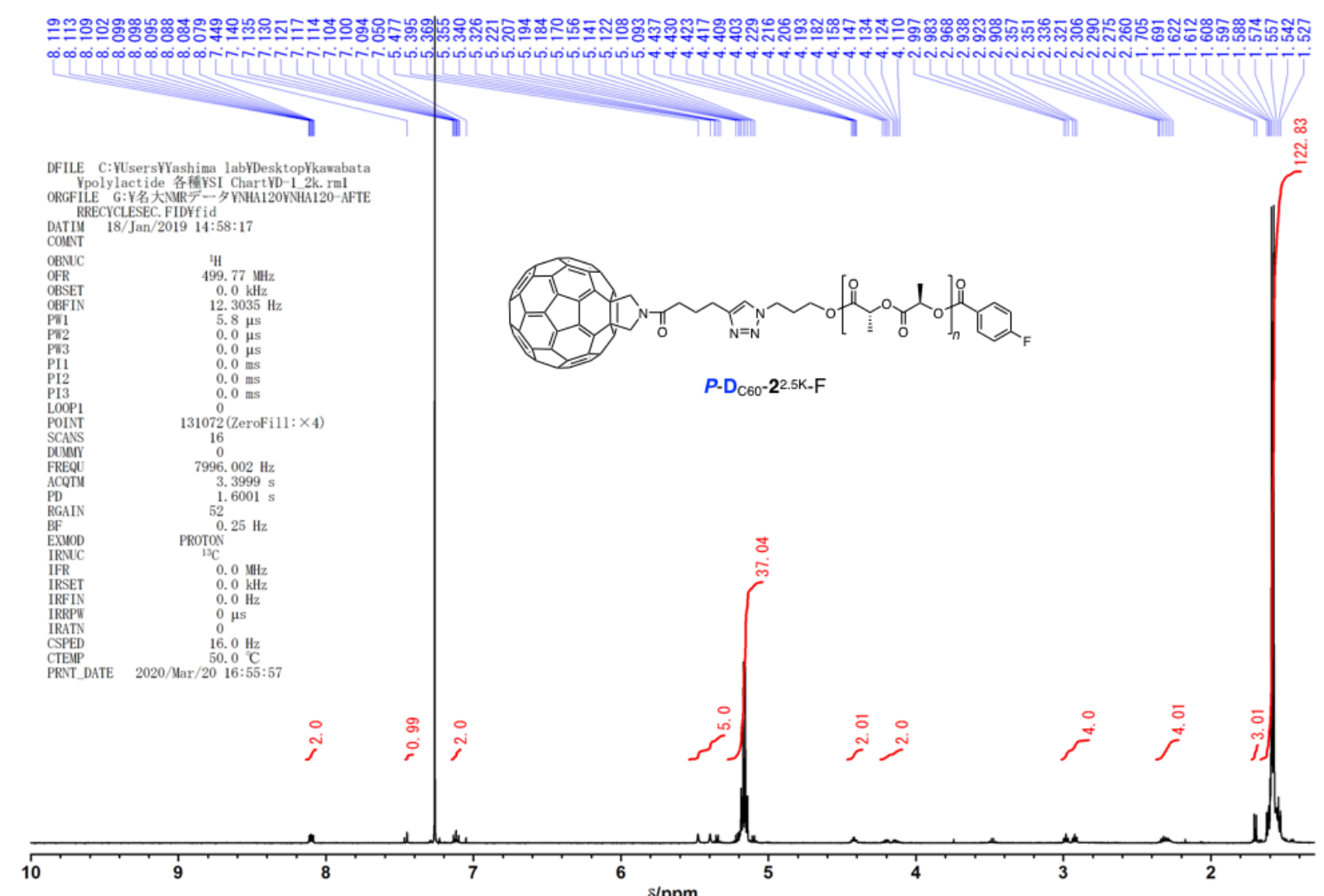

Figure S31. ${ }^{1} \mathrm{H}$ NMR $(500 \mathrm{MHz})$ spectrum of $\boldsymbol{P}-\mathbf{D}_{\mathrm{C} 60}-\mathbf{2}^{2.5 \mathrm{~K}_{-} \mathrm{F}}$ in $\mathrm{CDCl}_{3}$ at $25^{\circ} \mathrm{C}$. 


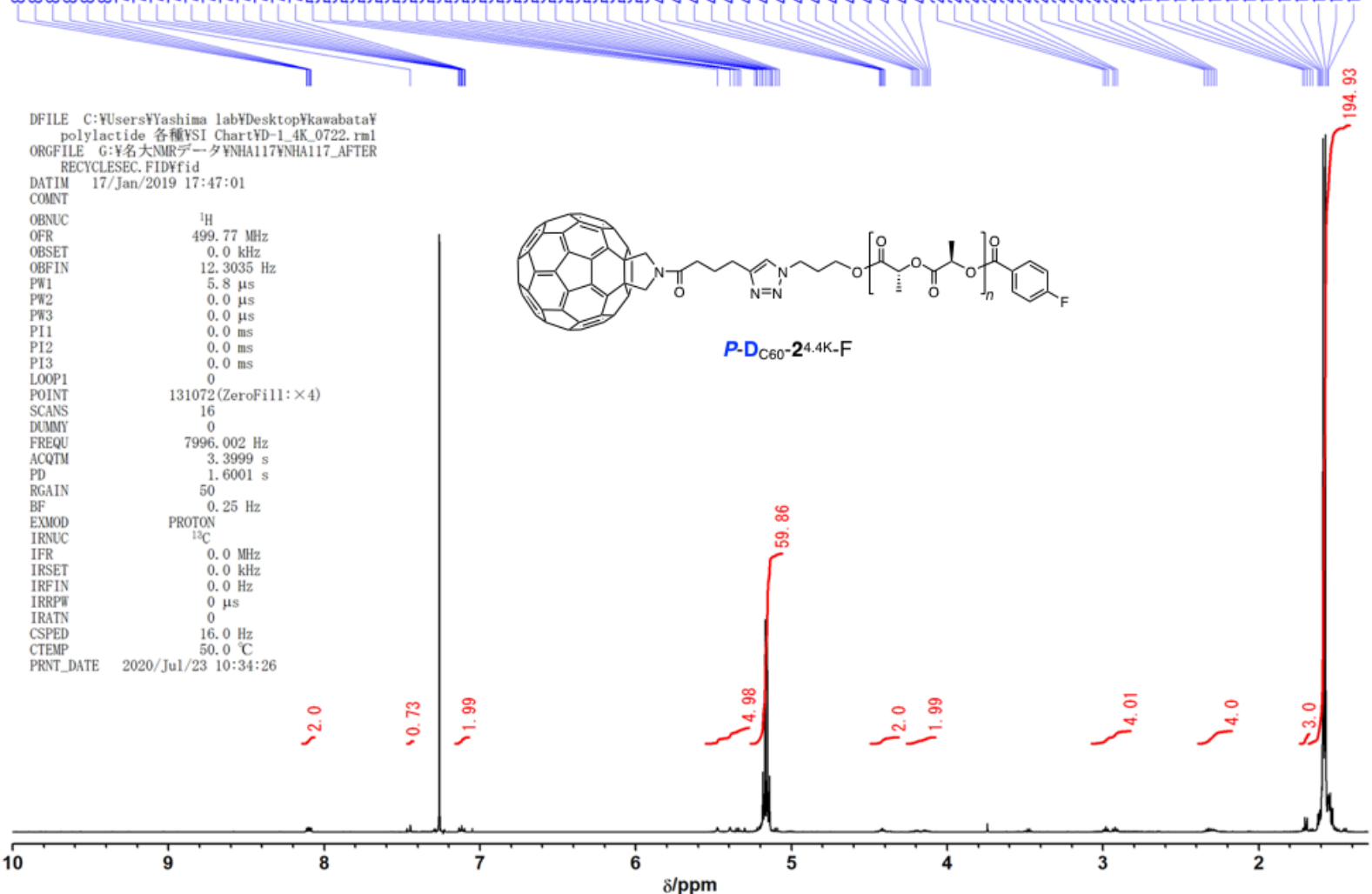

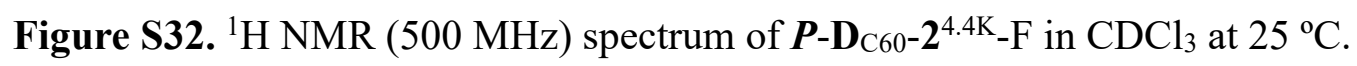

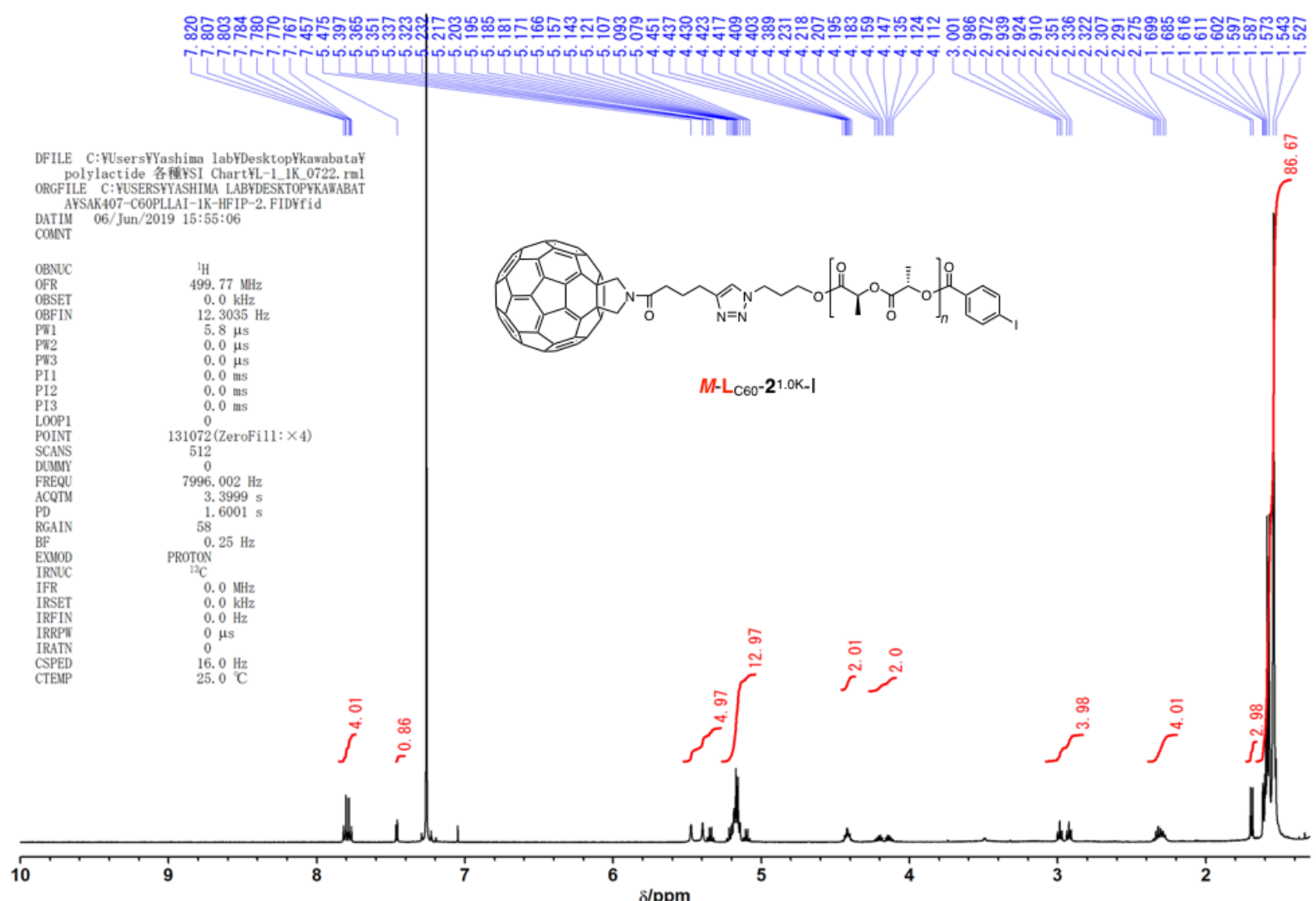

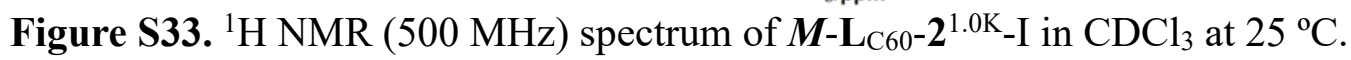




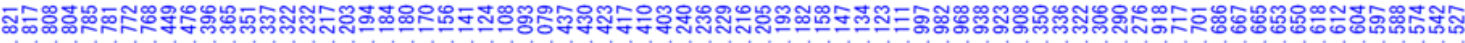

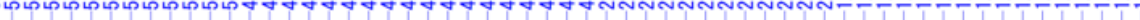

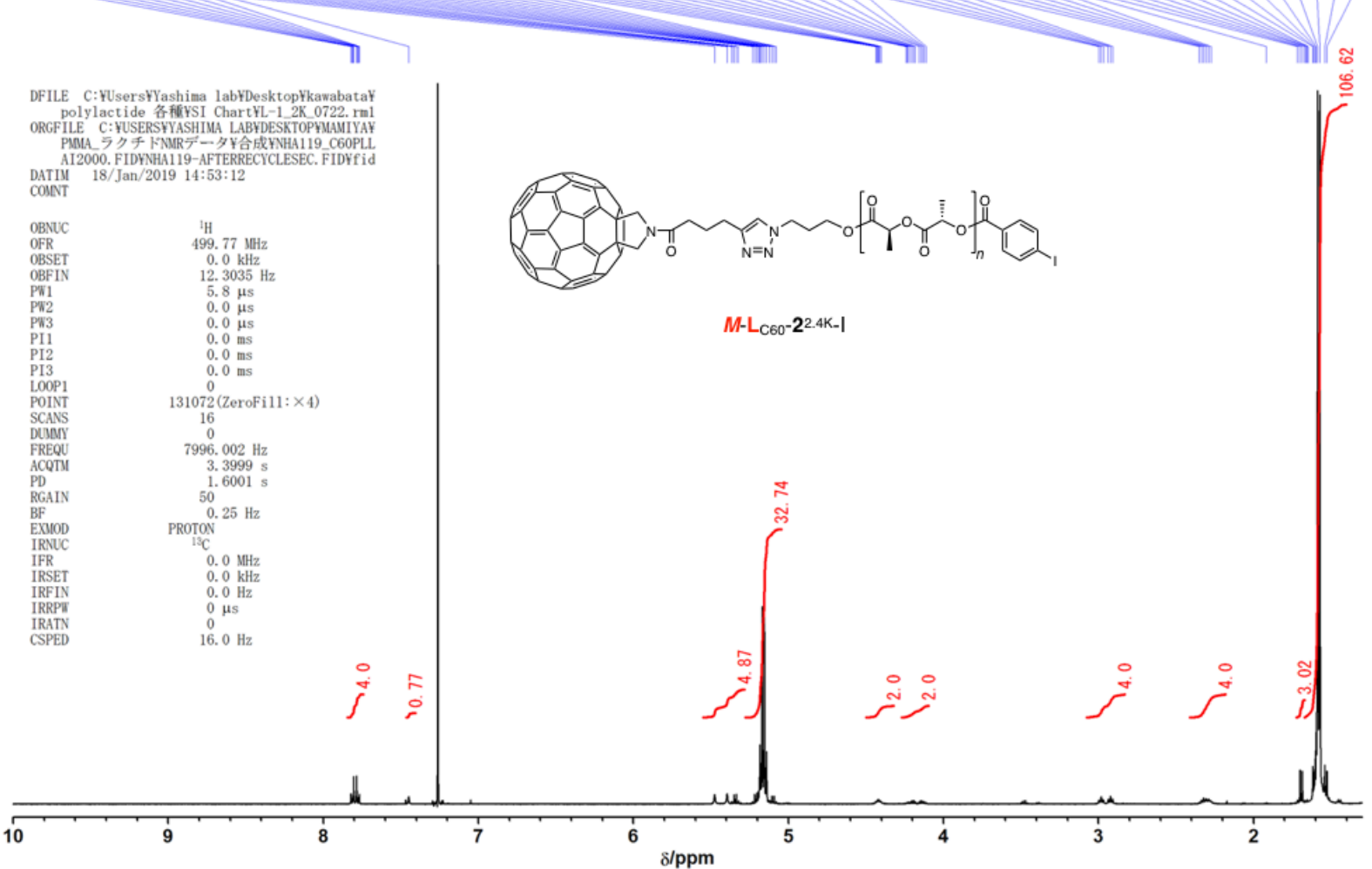

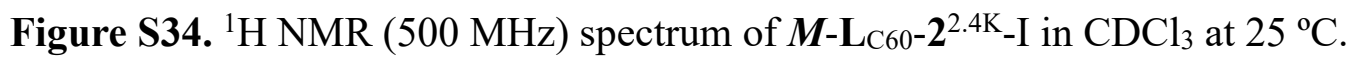

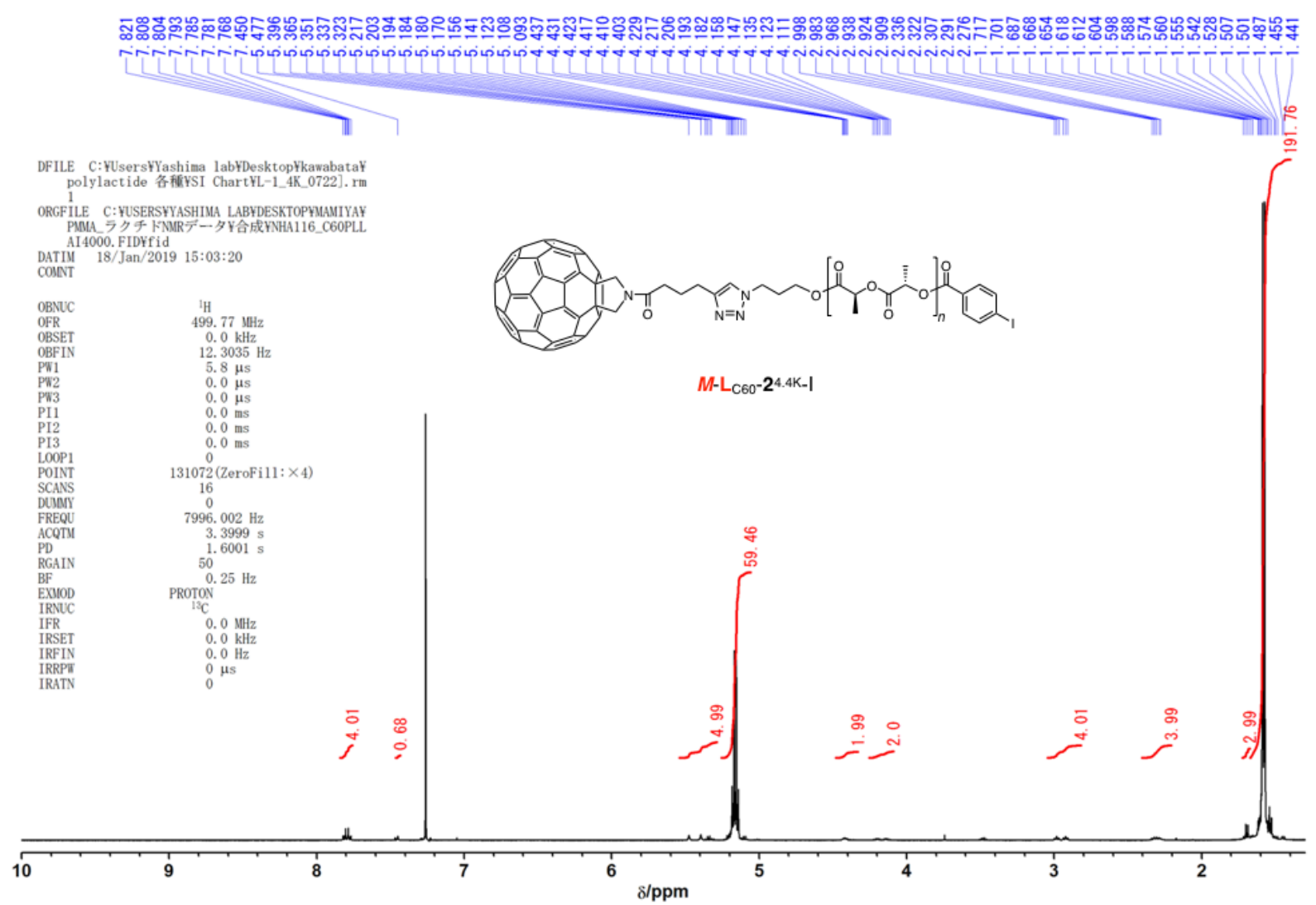

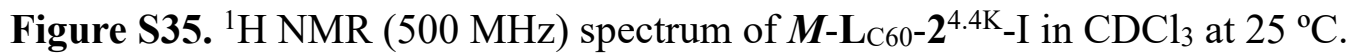

